HARMONIC ANALYSES OF STREAM TEMPERATURES

IN THE UPPER COLORADO RIVER BASIN

By Timothy Doak Steele

U.S. GEOLOGICAL SURVEY

Water-Resources Investigations Report 84-4290

Lakewood, Colorado

1985 
UNITED STATES DEPARTMENT OF THE INTERIOR

WILLIAM P. CLARK, Secretary

GEOLOGICAL SURVEY

Dallas L. Peck, Director

\author{
For additional information \\ write to: \\ Copies of this report can be \\ purchased from: \\ Open-File Services Section \\ Colorado District Chief \\ U.S. Geological Survey, MS 415 \\ Western Distribution Branch \\ U.S. Geological Survey, MS 306 \\ Box 25046, Denver Federal Center \\ Lakewood, CO 80225 \\ Box 25425, Federal Center \\ Denver, CO 80225 \\ Telephone: (303) 236-7476
}


Int roduction-1..- 1

Acknowledgments-1. 2

Method of analysis-_- 2

Stream-temperature analysis by subbasin-_. 7

White River subbasin-_. 7

Colorado River subbasin-_. 7

San Juan River subbasin-_. 9

Green River subbasin-_.- 21

Yampa River subbasin-_.-. 28

Colorado River at Lees Ferry, Ariz-_... 28

Impacts of reservoirs on water temperature-1. 36

Piceance basin-A specific study area- 38

Summary- 46

Selected references-1.- 47

Supplemental information: Water-temperature measurement-site

descriptions - 49

\section{Illustrations}

Figure 1. Map showing location of daily water-temperature measurement sites in the Upper Colorado River Basin-_. 6

2. Graphs showing stream-reach seasonal water-temperature patterns, White River, 1979 water year- 10

3. Graphs showing stream-reach seasonal water-temperature patterns, Colorado River, 1976 water year-ar 16

4. Graphs showing stream-reach seasonal water-temperature patterns, San Juan River, 1963 or 1964 water year--..

5. Graphs showing stream-reach seasonal water-temperature patterns, Green River, 1976 water year---

6. Graphs showing seasonal water-temperature patterns, Yampa River subbasin, 1979 water year-a 32

7. Graphs showing changes in seasonal water-temperature patterns, 1958 water year versus 1968 water year-_...- 34

8. Map showing location of water-temperature measurement sites, Piceance basin--..-

9. Graphs showing seasonal water-temperature patterns at selected sites, Piceance basin, 1979 water year-_- 


\section{CONTENTS}

\section{Tables}

Table 1. Inventory of available daily water-temperature records---_--- 4

2. Harmonic-function coefficients, White River subbasin-.-.-.-.- 8

3. Harmonic-function coefficients, Colorado River subbasin--.-.-- 11

4. Harmonic-function coefficients, San Juan River subbasin--.--.- 17

5. Harmonic-function coefficients, Green River subbasin--.-.-.- 22

6. Harmonic-function coefficients, Yampa River subbasin-_.....-. 30

7. Harmonic-function coefficients, Colorado River at Lees

Ferry, Ariz-... 33

8. Trend-analysis summary, long-term records, Upper Colorado

River Basin--..- 37

9. Harmonic-function coefficients, Piceance basin-_- 40

10. Water-temperature measurement-site descriptions-_- 50

\section{CONVERSION FACTORS AND RELATED INFORMATION}

For use of readers who prefer to use metric units, conversion factors for terms used in this report are listed below:
Multiply
By
To obtain
mile (mi)
1.609
kilometer
square mile $\left(\mathrm{mi}^{2}\right)$
2.590
square kilometer
Temperature in degrees Fahrenheit $\left({ }^{\circ} \mathrm{F}\right)$ can be converted to degrees Celsius $\left({ }^{\circ} \mathrm{C}\right)$ as follows:

$$
{ }^{\circ} \mathrm{F}=1.8^{\circ} \mathrm{C}+32
$$




\title{
HARMONIC ANALYSIS OF STREAM TEMPERATURES IN THE UPPER COLORADO RIVER BASIN
}

By Timothy Doak Steele ${ }^{1}$

\begin{abstract}
Harmonic analyses were made for available daily water-temperature records for 36 measurement sites on major streams in the Upper Colorado River Basin and for 14 measurement sites on streams in the Piceance basin. Generally ( 88 percent of the station years analyzed), more than 80 percent of the annual variability of temperatures of streams in the Upper Colorado River Basin was explained by the simple-harmonic function. Significant trends were determined for 6 of the 26 site records having long-term data ( 8 years or more). In most cases, these trends resulted from construction and operation of upstream surface-water impoundments occurring during the period of record. Regional analysis of water-temperature characteristics at the 14 streamflow sites in the Piceance basin, selected as a specific study area, indicated similarities in water-temperature characteristics for a small range of measurement-site elevations. Evaluation of information content of the daily records indicate that less-than-daily measurement intervals could result in substantial savings in measurement and data-processing costs.
\end{abstract}

\section{INTRODUCTION}

Stream temperature is one of the principal water-quality constituents of concern in water-resources planning and management, because of its dominant effect on aquatic life in streams. Moreover, water temperature influences waste-assimilation rates and is an important variable governing stream management for recreation or for municipal and industrial supplies.

As part of a regional evaluation of water-quality impacts of synfuels development in the Upper Colorado River Basin (Colorado Department of Natural Resources, 1979), available historical water-temperature records were analyzed using a simple-harmonic function to provide a quantitative description of the annual seasonal variability of stream temperatures. Recently renewed interest in constructing commercial-scale oil-shale conversion facilities in the

${ }^{1}$ Water Resources Manager, Engineering and Environmental Science Division, In-Situ, Inc., Lakewood, Colorado. 
Piceance basin resulted in a growing need to describe ambient conditions in that specific study area (including water-temperature characteristics), prior to extensive development in that area.

The purpose of this report is to summarize the information provided by many of the available daily water-temperature records in the Upper Colorado River Basin and the Piceance basin and to describe the general watertemperature conditions by major subbasin or specific study area. In this manner, a more objective rationale may evolve for justifying the existing ongoing regional-measurement program or for suggesting modifications to achieve a more efficient operating data-collection network.

\section{Acknowledgments}

Initial stages of this study were conducted under the auspices of the U.S. Geological Survey, Water Resources Division, Colorado District Office, Lakewood, Colo. Updated computer analyses were performed by Olivetree Consultants, Inc. Review comments by H. W. Lowham, U.S. Geological Survey, Cheyenne, Wyo., and T. R. Dyar, U.S. Geological Survey, Doraville, Ga., were most appreciated. R. B. Murphy and C. S. Curtis assisted in the final harmonic coefficient tabulations and time-trend analyses. Final computer analyses and preparation of this report were made possible by a research grant from the Professional Development Committee of Woodward-Clyde Consultants, Denver, Colo., and by In-Situ, Inc.

\section{METHODS OF ANALYSIS}

A computer program was used to perform harmonic analyses of daily (continuous) temperature records. A complete description of the program operations and of available options is given in a separate programdocumentation report (Steele, 1974). This harmonic-function model may be used to interpolate between discrete stream-temperature measurements or record gaps and to assess the effect of various measurement-frequency schemes (Gilroy and Steele, 1972). Resultant harmonic coefficients may be used for summarizing areal variations in annual seasonal stream-temperature characteristics and for detecting and evaluating changes in the coefficients over a period of several years.

In this computer program, a simple sine-function algorithm of the following form is used to define the annual seasonal cycle of water temperature at a streamflow site where stream temperature is measured (Ward, 1963; Collings, 1969):

$$
T(t)=A x[\sin (b t+C)]+M
$$

where: $T(t)=$ stream temperature, in degrees Celsius, on day $t$ of the annual time increment;

$A=$ amplitude of the harmonic, in degrees Celsius;

$b=0.0172$ radians per day $=(2 \pi \div 365$ or 366 days $) ;$

$C=$ phase angle of the harmonic, in radians; and

$M=$ mean of the harmonic, in degrees Celsius. 
With the above notation, stream temperature, $\mathrm{T}$, at a specified site is denoted to vary as a function of time, $t$. Unless annual stream-temperature characteristics are affected appreciably by economic activity in the catchment upstream from a sampling site, the harmonic coefficients (,$C$, and $M$ ) are considered to be constant in time (unless a trend is observed), and to vary principally as a function of location.

The rationale for applying a first-order (simple) harmonic function is discussed by Steele (1978), in addition to demonstrating several capabilities of the computer program. Of the harmonic coefficients (,$C$, and $M$ ), $A$ and $M$ are of principal concern. Areal variations for $C$ (which indicates the phasing of the seasonal cycle for a specified annual increment) generally are small compared with those for $A$ and $M$, because of its primary dependence upon hemispheric-climatic patterns. These latter coefficients are affected appreciably by such environmental factors as station latitude, altitude, vegetative cover, exposure, and dominant origin of the water (that is, the proportion of ground water contributing to streamflow).

The computer program makes appropriate adjustments for missing values or gaps in a record. However, no attempt is made in this model to account for diel temperature fluctuations in the input-data records; rather, these fluctuations are aggregated with other unaccounted-for noise in the data in the standard error of estimate. Choice of the annual time-increment will affect harmonic coefficient $C$ of equation 1 ; the water year (October 1 of year n-1 through September 30 of year $n$ ) was used throughout this study. The mode1 ignores data values at or below freezing $\left(0^{\circ} \mathrm{C}\right)$ temperature (Steele, 1974).

Use of the harmonic-analysis technique for describing water-temperature characteristics has been demonstrated in several studies (Shampine, 1977; Clement, 1978; Lowham, 1978; Wentz and Steele, 1980). Moreover, this procedure has been applied to station records in the U.S. Geological Survey's National Stream Quality Accounting Network (Hawkinson and others, 1977; Briggs and Ficke, 1978; Steele, 1983). For station records of sufficient duration (assumed in this study to be 8 years or more), statistical tests of time trends may be made, using a procedure documented by Conover (1971) and applied by Steele and others (1974).

For main-stem rivers, upstream-downstream profiles of water-temperature harmonic coefficients serve to characterize conditions along the stream reach. Sample case studies are described by Lowham (1978) for the Green River and by Steele and others (1979) for the Yampa River and the Little Snake River.

For purposes of the Upper Colorado River Basin analysis, a total of 751 years of daily water-temperature records at 50 sites were retrieved from the U.S. Geological Survey's WATSTORE data files (Hutchinson, 1975). These records are inventoried in table 1 . Station descriptions are given in table 10 in the Supplemental Data section at the end of this report. For the 36 sites in the Upper Colorado River Basin (fig. 1) outside of the Piceance basin, the periods of available record ranged from 2 to 35 years and averaged nearly 19 years for 36 sites. Several records are discontinuous in time, with one or more years of missing record (table 1). Moreover, most daily records fitted to a harmonic-function by annual increments have a number of missing values for a given year. Hence, the annual season-cycle characterization 


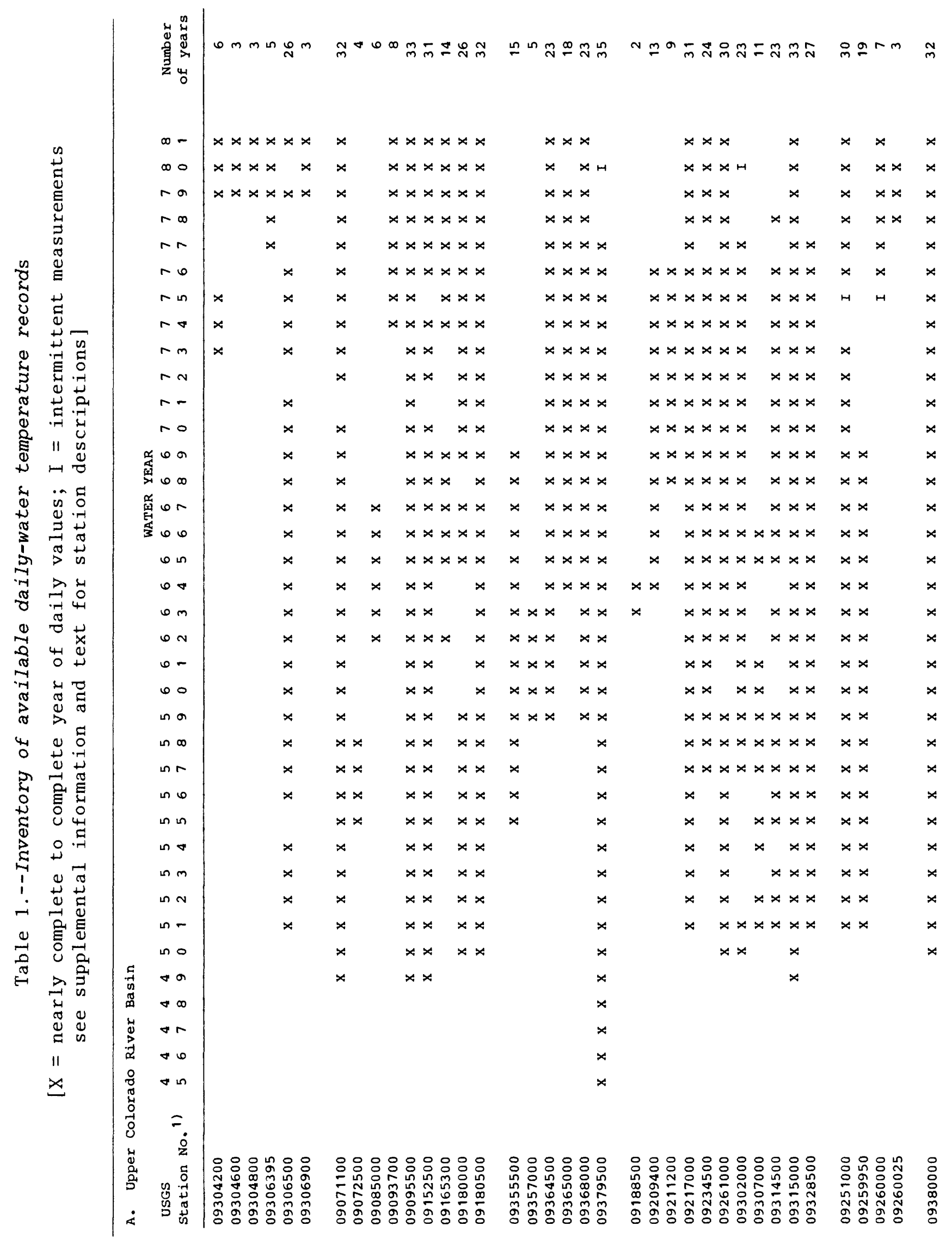


Table 1.--Inventory of available daily water-temperature records--Continued

B. Piceance basin $\begin{array}{lllllllll}\text { USGS } & 7 & 7 & 7 & 7 & 7 & 8 & 8 & \text { Number }\end{array}$

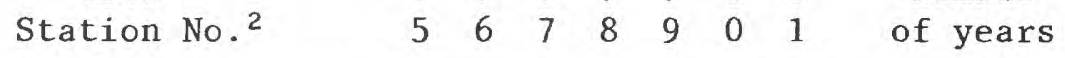

\begin{tabular}{|c|c|c|c|c|c|c|c|c|}
\hline 90902850 & & & $\mathrm{X}$ & $\mathrm{X}$ & $\mathrm{X}$ & $\mathrm{X}$ & $\mathrm{X}$ & 5 \\
\hline 09093000 & $\mathrm{X}$ & $\mathrm{X}$ & $\mathrm{X}$ & $\mathrm{X}$ & $\mathrm{X}$ & $\mathrm{X}$ & & 6 \\
\hline 09093500 & $X$ & $\mathrm{X}$ & $\mathrm{X}$ & $\mathrm{X}$ & $\mathrm{X}$ & $\mathrm{X}$ & & 6 \\
\hline 09095000 & $\mathrm{X}$ & $\mathrm{X}$ & $\mathrm{X}$ & $\mathrm{X}$ & $\mathrm{X}$ & $\mathrm{X}$ & & 6 \\
\hline 09306007 & $\mathrm{X}$ & $\mathrm{X}$ & $\mathrm{X}$ & $\mathrm{X}$ & $\mathrm{X}$ & $\mathrm{X}$ & $\mathrm{X}$ & 7 \\
\hline 09306022 & $\mathrm{X}$ & $\mathrm{X}$ & $\mathrm{X}$ & $\mathrm{X}$ & $\mathrm{X}$ & $\mathrm{X}$ & $\mathrm{X}$ & 7 \\
\hline 09306025 & $\mathrm{X}$ & $\mathrm{X}$ & & & & & & 2 \\
\hline 09306058 & $\mathrm{X}$ & $\mathrm{X}$ & $\mathrm{X}$ & $\mathrm{X}$ & $\mathrm{X}$ & $\mathrm{X}$ & $\mathrm{X}$ & 7 \\
\hline 09306061 & $\mathrm{X}$ & $\mathrm{X}$ & $\mathrm{X}$ & $\mathrm{X}$ & $x$ & $\mathrm{X}$ & $\mathrm{X}$ & 7 \\
\hline 09306175 & $\mathrm{X}$ & $\mathrm{X}$ & $\mathrm{X}$ & $X$ & $\mathrm{X}$ & $\mathrm{X}$ & $\mathrm{X}$ & 7 \\
\hline 09306222 & & $\mathrm{X}$ & $\mathrm{X}$ & $\mathrm{X}$ & $\mathrm{X}$ & $\mathrm{X}$ & $\mathrm{X}$ & 6 \\
\hline 09306235 & & & $\mathrm{X}$ & $\mathrm{X}$ & $\mathrm{X}$ & $\mathrm{X}$ & $\mathrm{X}$ & 5 \\
\hline 09306242 & $\mathrm{X}$ & & $\mathrm{X}$ & $\mathrm{X}$ & $X$ & $\mathrm{X}$ & $\mathrm{X}$ & 6 \\
\hline 09306255 & $\mathrm{X}$ & $\mathrm{X}$ & $\mathrm{X}$ & $X$ & $\mathrm{X}$ & $X$ & $\mathrm{X}$ & 7 \\
\hline
\end{tabular}

1See supplemental information and text for station descriptions; sites located in figure 1.

${ }^{2}$ See supplemental information and text for station descriptions; sites located in figure 8 .

of water temperature by the harmonic function is a means of standardizing the information contained in the data records of different measurement frequencies, or data records with missing values and gaps (Gilroy and Steele, 1972; Clement, 1978).

In the Piceance basin specific study area, 83 years of daily watertemperature data were available at 14 sites (table 1). Data collection for daily stream temperatures in the Piceance basin generally began in mid-1974, at the earliest. Hence, no more than 7 years of record was available at any given site, and the average period of record was about 6 years for the 14 sites.

Graphic and tabular results of the harmonic analysis are given by subbasin or specific study area (in the case of the Piceance basin). Selected harmonic-function curves are presented, along with stream-reach profiles for main-stem rivers for a particular year of record. Where applicable, causes for significant time trends in annual water-temperature characteristics are discussed. Finally, several observations of the continuing need for daily water-temperature records are summarized, with recommendations for achieving a more user-responsive and cost-effective operating water-temperature network. 


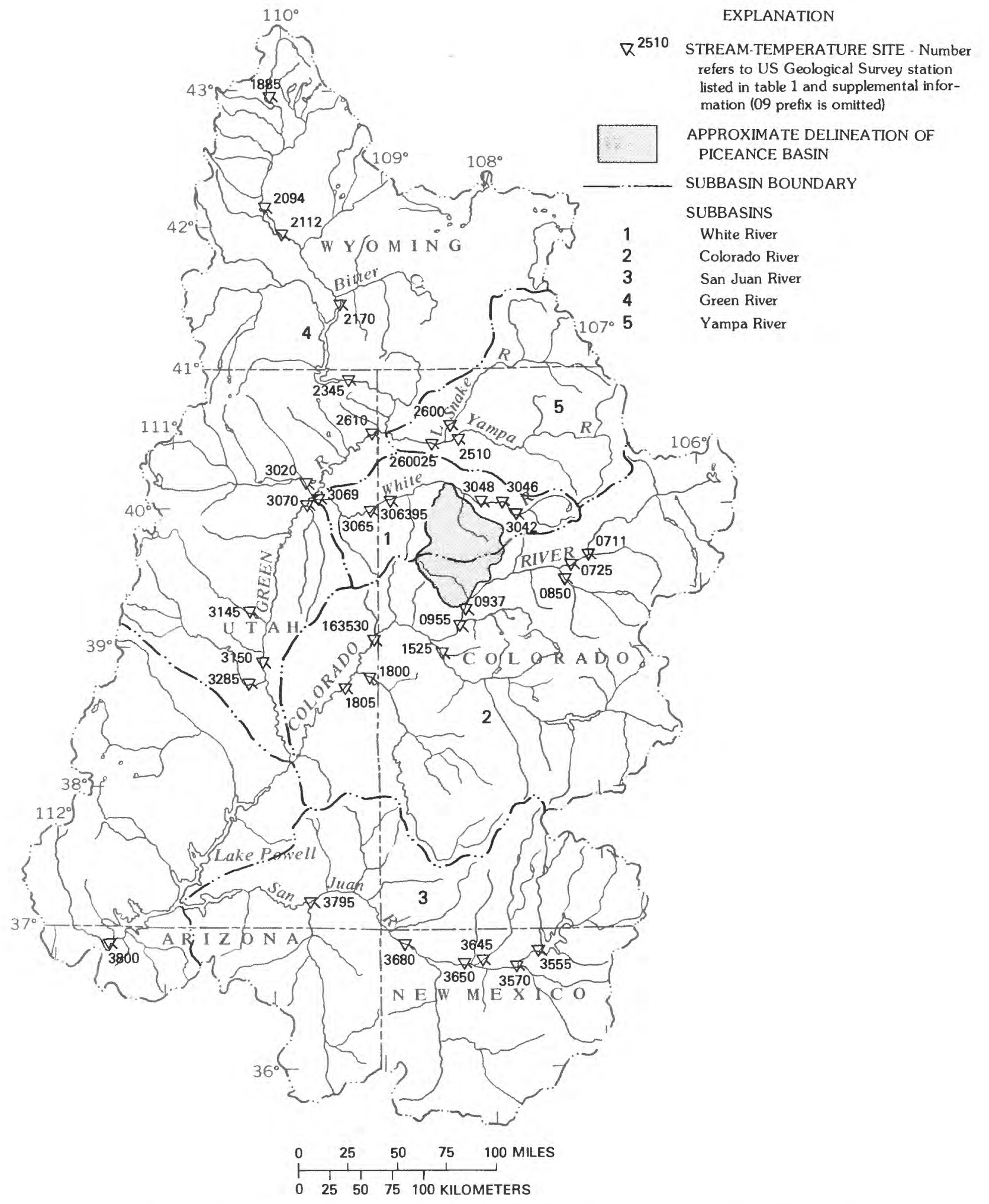

Figure 1.--Location of daily water-temperature measurement sites in the Upper Colorado River Basin. 


\section{STREAM-TEMPERATURE ANALYSIS BY SUBBASIN}

Results of the harmonic analyses of water-temperature records will be discussed by major subbasin (fig. 1). The sequence order corresponds with the critical-area ordering described by the Colorado Department of Natural Resources (1979). For demonstrating the seasonal cyclical pattern of water temperatures at several sites along a stream reach, harmonic functions for a typical annual increment are displayed graphically. Normally, a year has been selected in which daily records are available for the greatest number of main-stem sampling sites, or sites in a given subbasin or region. For purposes of assessing time trends in the annual time series for harmonic coefficients, a ranking procedure initially proposed and applied by Steele and others (1974) was used. The Kendell's tau ranking test (Conover, 1971) is included in this procedure. Most of the discussion of time trends is deferred to the section on impacts of reservoirs on water temperature.

\section{White River Subbasin}

Daily water-temperature data were analyzed for 46 station-years of record at six sites, all located on the main-stem White River (see table 1 and table 10 in the supplemental information section at the end of the report). Harmonic-function coefficients are summarized in table 2. The longest period of record in this subbasin consists of 26 years of record at station 09306500 White River near Watson, Utah. As has been noted in previous studies in nearby regions (Lowham, 1978; Steele and others, 1979), the harmonic mean temperatures increase in a downstream direction from $7.1^{\circ} \mathrm{C}$ for station 09304200 White River above Coal Creek near Meeker, Colo., to $11.1^{\circ} \mathrm{C}$ for station 09306900 White River at mouth near Ouray, Utah (fig. 2), using data for the 1979 water year (table 2). Also, for the White River, the harmonic amplitude nearly doubles over the same reach of stream. Significant time trends were detected in the annual time series of the mean-plus-amplitude coefficients for station 09306500 White River near Watson, Utah.

\section{Colorado River Subbasin}

A total of 186 years of daily water-temperature records is available at nine sites in the Colorado River subbasin. Six of these sites are located on the main-stem Colorado River (see table 10 in the supplemental information section at the end of the report). The other three are major tributaries in the subbasin. Harmonic-function coefficients for each station-year of record have been tabulated (table 3). Using coefficients for the 1976 water year, mean temperatures in the Colorado River increase from $9.1^{\circ} \mathrm{C}$ at station 09071100 Colorado River near Glenwood Springs, Colo., to over $11.9^{\circ} \mathrm{C}$ at station 09163530 Colorado River below Colorado-Utah State line, and $11.6^{\circ} \mathrm{C}$ station 09180500 Colorado River near Cisco, Utah. However, the 1976 mean temperature is slightly greater than expected at DeBeque $\left(10.2^{\circ} \mathrm{C}\right.$ for 1976 at station 09093700 Colorado River near De Beque, Colo.) for an increasing stream-reach trend in a downstream direction. For the periods of record, harmonic amplitudes range from 7.9 to $11.5^{\circ} \mathrm{C}$ at station 09163530 Colorado River below Colorado-Utah State line. Stream-reach patterns during the 
Table 2.--Harmonic-function coefficients, White River subbas in

[NO = number of daily values; $\mathrm{SE}=$ standard error of estimate; PVAR = percent variance explained by the harmonic function]

\begin{tabular}{|c|c|c|c|c|c|c|c|}
\hline \multirow[b]{2}{*}{ Station no. ${ }^{2}$} & \multirow[b]{2}{*}{ Year } & \multicolumn{4}{|c|}{ Harmonic coefficients ${ }^{1}$} & \multirow[b]{2}{*}{$\mathrm{SE}$} & \multirow[b]{2}{*}{ PVAR } \\
\hline & & NO & A & C & M & & \\
\hline 9304200 & 1973 & 187 & 5.83 & 2.40 & 11.11 & 1.77 & 78 \\
\hline 9304200 & 1974 & 355 & 7.18 & 2.81 & 10.08 & 1.99 & 86 \\
\hline $930+200$ & 1975 & 351 & 7.30 & $2+68$ & 6.29 & 1.90 & 83 \\
\hline 9304200 & 1579 & 173 & 0.87 & 2.81 & 7.07 & 1.30 & 99 \\
\hline 7304200. & 1980 & 229 & 7.86 & 2.77 & $b .81$ & 1.54 & 99 \\
\hline 7304200 & 1981 & 350 & 7.85 & 2.84 & 7.59 & $1 \cdot 28$ & 88 \\
\hline 9304600 & 1979 & 299 & 7.63 & 2.79 & 7.44 & 1.77 & 95 \\
\hline 9304600 & 1980 & 274 & 5.21 & 2.95 & 8.36 & 1.62 & 99 \\
\hline 9304600 & 1981 & 343 & 7.62 & 2.83 & 8.45 & 1.71 & 35 \\
\hline 9304800 & 1979 & 343 & 7.99 & 2.77 & 8.15 & 1.93 & 86 \\
\hline 9304800 & 1980 & 310 & 8.65 & 2.76 & 8.26 & 1.97 & $B 8$ \\
\hline 9304800 & 1981 & 350 & 9.05 & 2.87 & 8.64 & 1.69 & 91 \\
\hline 9306395 & 1977 & 191 & 13.22 & 2.93 & 10.13 & 1.70 & 99 \\
\hline 9306395. & 1978 & 265 & 11.05 & 2.67 & 10.34 & $2 \cdot 67$ & 99 \\
\hline 9306395 & 1979 & 272 & 11.99 & 2.72 & 9.63 & 2.23 & 99 \\
\hline 9306395 & 1980 & 275 & $12 \cdot 10$ & 2.80 & 9.98 & 2.10 & 99 \\
\hline 9306395 & 1981 & 365 & 11.51 & 2.90 & 11.24 & 1.93 & 94 \\
\hline 9306500 & 1951 & 253 & 10.92 & 2.70 & 10.79 & 2.27 & 92 \\
\hline 5306500 & 1952 & 311 & 13.08 & 2.63 & 9.53 & 2.94 & 98 \\
\hline 9306500. & 1953 & 300 & 12.09 & 2.69 & 10.02 & 2.38 & 99 \\
\hline 9306500 & 1954 & 289 & 12.13 & 2.77 & 11.01 & 2.64 & 89 \\
\hline 9306500 & 1956 & 318 & 11.51 & 2.67 & 11.26 & 2.58 & 70 \\
\hline 9306500 & 1957 & 362 & 9.72 & 2.63 & 5.00 & 2.19 & 89 \\
\hline 9306500 & 1958 & 299 & 10.46 & 2.60 & 9.23 & 2.64 & 86 \\
\hline 7306500. & 1959 & 300 & 10.46 & 2.87 & 10.93 & 2.39 & 90 \\
\hline 9306500 & 1960 & 316 & 10.36 & 2.79 & 9.58 & 2.16 & 91 \\
\hline 9306500. & 1961 & 247 & 12.21 & 2.87 & 7.96 & 2.26 & 90 \\
\hline 9306500 & 1962 & 231 & 10.83 & 2.81 & 9.03 & 2.33 & 87 \\
\hline 9306500 & 1963 & 259 & 12.24 & 2.81 & 10.01 & 2.02 & 92 \\
\hline 9306500. & 1964 & 215 & 12.76 & 2.65 & 8.93 & 2.25 & $B B$ \\
\hline 9306500. & 1965 & 166 & $11+24$ & 2.68 & 8.09 & 2.39 & 82 \\
\hline 5306500 & 1966 & 225 & $11 \cdot 13$ & 2.82 & 9.94 & 1.88 & 93 \\
\hline 9300500 & 1967 & 248 & 10.76 & 2.72 & 9.70 & 2.31 & 87 \\
\hline 5306500 & 1568 & 237 & 10.53 & $2+79$ & 8.11 & 2.07 & 86 \\
\hline 5306500 & 1969 & 267 & 10.98 & 2.78 & 9.10 & 2.14 & 89 \\
\hline 934600 & 1970 & 205 & 7.02 & 2.75 & 6.76 & 2.23 & 78 \\
\hline 9306500. & $\$ 571$ & 317 & 9.90 & 2.70 & 2.36 & $2 \cdot 23$ & 90 \\
\hline 93065010 & 1973 & $=97$ & $2 \%, 30$ & $2.6 \theta$ & 7.58 & 2.02 & $8 \Xi$ \\
\hline 930,6000 & 1574 & 148 & 11.35 & 2.77 & 7.64 & 2.02 & 90 \\
\hline 930.6500 & 1775 & 102 & 10.37 & $2+t 7$ & 7.77 & 2.56 & 81 \\
\hline
\end{tabular}


Table 2.--Harmonic-function coefficient, white River subbasin--Continued

\begin{tabular}{lcrrrrrr}
\hline & \multicolumn{7}{c}{ Harmonic coefficients } \\
Station no. & Year & NO & A & C & M & SE & PVAR \\
\hline 9306500. & 1976 & 135 & 11.88 & 2.82 & 9.38 & 2.15 & 93 \\
9306500. & 1979 & 92 & 10.19 & 2.66 & 10.14 & 2.52 & 76 \\
9306500. & 1981 & 90 & 7.49 & 2.26 & 11.58 & 2.07 & 75 \\
9306900. & 1979 & 105 & 12.29 & 2.87 & 11.10 & 2.23 & 72 \\
9306900. & 1980 & 244 & 13.01 & 2.89 & 10.69 & 1.86 & 84 \\
9306900. & 1981 & 331 & 12.02 & 2.91 & 11.57 & 1.82 & 87 \\
\hline
\end{tabular}

${ }^{1}$ See equation (1), p. 2 of text.

${ }^{2}$ See supplementary information for station description

1976 water year are depicted in figure 3. A general increasing stream-reach trend is observed, on the average, for the six main-stem sites. Mean temperatures at station 09085000 Roaring Fork River near Glenwood Springs, Colo., appears to be slightly greater than at station 09072500 Colorado River at Glenwood Springs, Colo., where the Roaring Fork River flows into the Colorado River. For the six long-term records, significant time trends in the annual series of harmonic coefficients were detected only for the harmonic mean temperature for the Colorado River at station 09095500 Colorado River near Cameo, Colo., using the Kendall's tau test (Conover, 1971).

\section{San Juan River Subbasin}

A total of 119 station years of daily water-temperature data was available for five main-stem sites and one major tributary (Animas River) in the San Juan River subbasin. Harmonic-function coefficients are given in table 4. Beginning in 1963, the San Juan River has been regulated by the Navajo Reservoir upstream from Archuleta, N. Mex. at station 09355500 (San Juan River near Archuleta, N. Mex.). Because initial regulation by this reservoir occurred about midway in the historical record at this nearby site, the impacts of streamflow regulation on downstream seasonal-temperature patterns could be readily assessed. In a comparison of harmonic coefficients for the 1963 and 1964 water years at the five main-stem sites, meantemperature patterns in the stream reach of the San Juan River followed patterns observed for the Colorado River; however, overall temperatures were about $3.0^{\circ} \mathrm{C}$ greater for the San Juan River relative to the Colorado River. Both mean and amplitude coefficients depicting water-temperature patterns of the San Juan River increased in a downstream direction from station 09355500 San Juan River near Archuleta, N. Mex., to station 09368000 San Juan River at Shiprock, N. Mex. (fig. 4). However, anomalously high coefficients were observed for station 09357000 San Juan River at Bloomfield, N. Mex., for the 1963 water-year record. However, mean water temperatures at station 09364500 

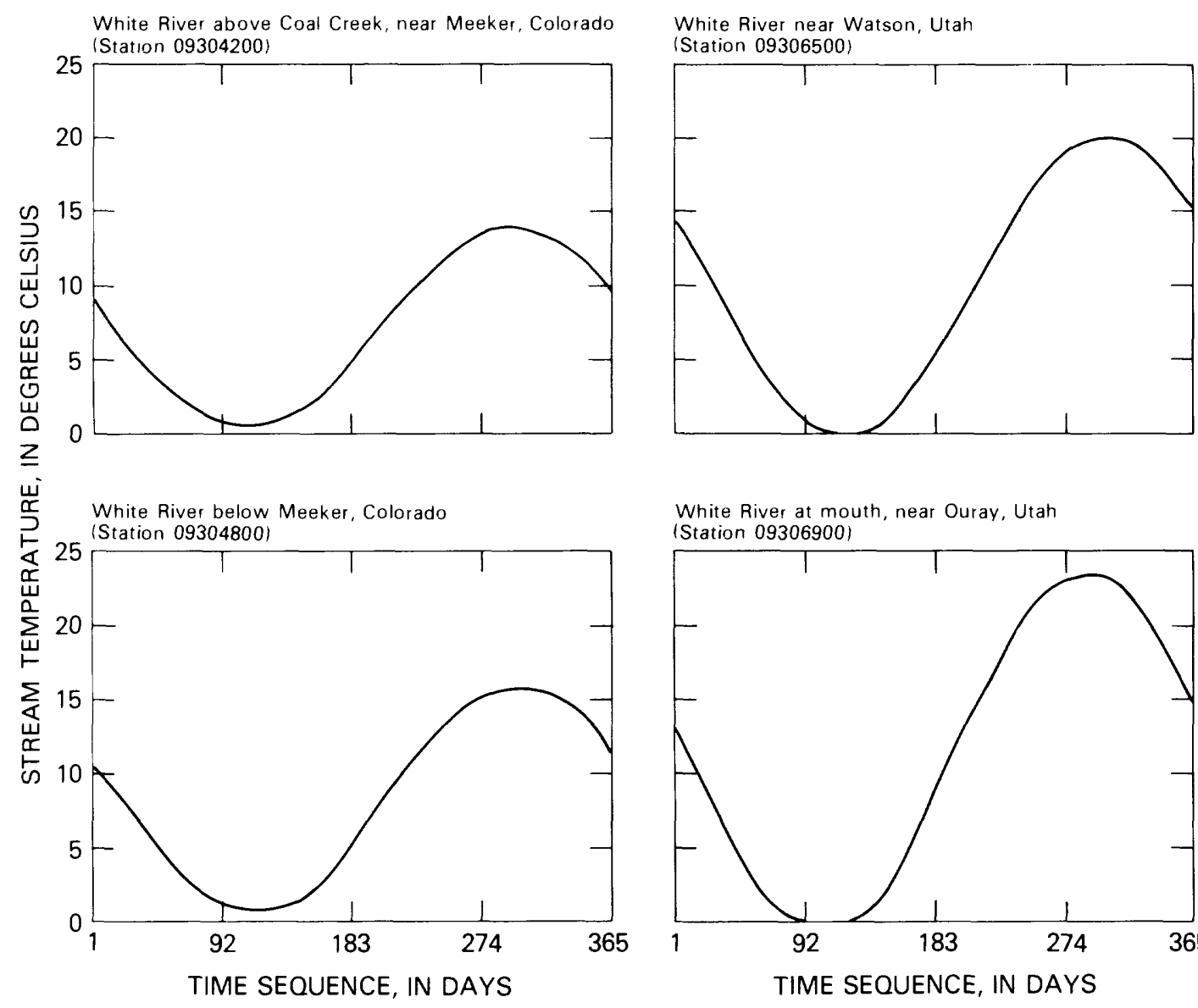

White River at mouth, near Ouray, Utah (Station 09306900)

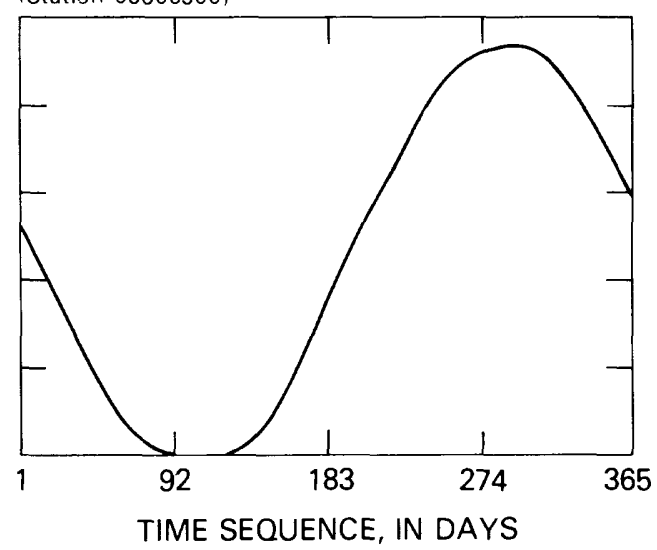

Figure 2.--Stream-reach seasonal water-temperature patterns, White River, 1979 water year. 
Table 3.--Harmonic-function coefficients, Colorado River subbas in

[NO = number of daily values; $\mathrm{SE}=$ standard error of estimate;

PVAR = percent variance explained by the harmonic function]

\begin{tabular}{|c|c|c|c|c|c|c|c|}
\hline \multirow[b]{2}{*}{ Station no. ${ }^{2}$} & \multirow[b]{2}{*}{ Year } & \multicolumn{4}{|c|}{ Harmonic coefficients ${ }^{1}$} & \multirow[b]{2}{*}{$\mathrm{SE}$} & \multirow[b]{2}{*}{ PVAR } \\
\hline & & NO & A & C & M & & \\
\hline 9071100 & 1949 & 153 & 7.85 & 2.42 & 9.22 & 1.56 & 75 \\
\hline 9071100 & 1950 & 364 & 7.74 & 2.06 & 8.72 & 1.53 & 92 \\
\hline 9071100 & 1951 & 355 & 7.97 & 2.67 & 8.50 & 1.61 & 92 \\
\hline 9071100 & 1952 & 307 & 8.64 & 2.66 & 7.90 & 1.89 & 90 \\
\hline 9071100 & 1953 & 272 & 8.61 & 2.72 & 7.92 & 1.69 & 90 \\
\hline 9071100 & 195.4 & 303 & 9.87 & 2.83 & 9.02 & 1.74 & 92 \\
\hline 9071100 & 1955 & 289 & 9.83 & 2.70 & 8.25 & 1.64 & 93 \\
\hline 9071100 & 1956 & 305 & 9.09 & 2.77 & 8.47 & 1.54 & 94 \\
\hline 9071100 & 1957 & 273 & 7.48 & 2.76 & $7 \cdot 34$ & 2.12 & 81 \\
\hline 9071100 & 1958 & 276 & 8.62 & 2.70 & 7.72 & 1.85 & 88 \\
\hline 0,071100 & 1959 & 286 & 8.97 & 2.79 & 8. 17 & 1.77 & 90 \\
\hline 9071100 & 1960 & 269 & 9.41 & 2.79 & 7.64 & 1.97 & 87 \\
\hline 9071100 & 1961 & 270 & 9.53 & 2.83 & 8.31 & 2.00 & 88 \\
\hline 9071100 & 1962 & 264 & 8.39 & 2.71 & 7.16 & 1.85 & 86 \\
\hline 9071100 & 1963 & 294 & 9.60 & 2.72 & 8.68 & 1.35 & 95 \\
\hline 9071100 & 1964 & 216 & 9.75 & $2.6 \overline{3}$ & 7.44 & 1.71 & 87 \\
\hline 9071100 & 1965 & 275 & 7.60 & 2.74 & 7.05 & 1.67 & 89 \\
\hline 9071100 & 1766 & 275 & $9+28$ & 2.76 & 8.55 & 1,81 & 91 \\
\hline 9071100 & 1967 & 313 & 8.39 & 2.75 & 8.08 & 1.75 & 91 \\
\hline 9071100 & 1968 & 299 & 8.55 & 2.80 & 7.40 & 1.69 & 91 \\
\hline 9071100 & 1969 & 291 & 9.05 & 2.79 & 8.32 & 2.11 & 88 \\
\hline 9071100 & 1970 & 317 & 8.65 & 2.76 & 7.55 & 2.02 & 89 \\
\hline 9071100 & 1972 & 353 & 8.64 & 2.82 & 9.68 & 1.86 & 91 \\
\hline 9071100 & 1973 & 356 & 8.35 & 2.74 & 9.18 & 1.64 & 92 \\
\hline 9071100 & 1974 & 356 & 8.66 & 2.73 & 9.09 & 1.54 & 74 \\
\hline 9071100. & 1975 & 365 & 8.27 & 2,74 & 8.77 & 1.61 & 92 \\
\hline 7071100 & $197 \mathrm{~b}$ & 339 & 9.19 & 2.77 & 9.08 & 1.73 & 93 \\
\hline 9071100 & 1977 & 357 & 9.91 & 2.82 & 9.50 & 1.49 & 95 \\
\hline 9071100 & 1979 & 365 & 7.95 & 2.78 & 9.58 & 1.93 & 83 \\
\hline 9071100 & 1975 & 365 & 0.05 & $2+75$ & 8.95 & 1.91 & 89 \\
\hline 7071100 & 1980 & 218 & 8.46 & 2.36 & 9.69 & 1.84 & 84 \\
\hline 5071100. & 1981 & 364 & 10.52 & 2.88 & 10.98 & 1.82 & 93 \\
\hline 9072500 & 1955 & 290 & 9.79 & 2.70 & 8.30 & 1.65 & 93 \\
\hline 9072500 & 1950 & 306 & 8.96 & 2.78 & 8.43 & 1.53 & 94 \\
\hline 7072500 & 1957 & 273 & 7.61 & 2.75 & 7.24 & 1.94 & 84 \\
\hline 7072500 & $195 \bar{\theta}$ & 276 & 8.74 & 2.70 & 7.66 & 1.73 & 90 \\
\hline 9085000. & 1962 & 145 & 7.43 & 2.27 & 9.41 & 1.45 & 77 \\
\hline $908500 \%$ & 1763 & 3.57 & 8.36 & 2.76 & 10.41 & 1.97 & 89 \\
\hline 7085000 & 1964 & 361 & 8.32 & 2.65 & 9.75 & 2.00 & 89 \\
\hline
\end{tabular}


Table 3.--Harmonic-function coefficients, Colorado River subbasin--Continued

\begin{tabular}{|c|c|c|c|c|c|c|c|}
\hline \multirow[b]{2}{*}{ Station no. ${ }^{2}$} & \multirow[b]{2}{*}{ Year } & \multicolumn{4}{|c|}{ Harmonic coefficients ${ }^{1}$} & \multirow[b]{2}{*}{$\mathrm{SE}$} & \multirow[b]{2}{*}{ PVAR } \\
\hline & & NO & A & $\mathrm{C}$ & M & & \\
\hline 9085000 & 1965 & 363 & 6.56 & 2.73 & 9.50 & 2.24 & 81 \\
\hline 9085000 & 1966 & 362 & 8.62 & 2.74 & 10.55 & 1.84 & 91 \\
\hline 9085000 & 1967 & 354 & 7.58 & 2.75 & 10.11 & 2.27 & 84 \\
\hline 9093700 & 1974 & 299 & 9.39 & 2.73 & 10.08 & 1.90 & 90 \\
\hline 9093700 & 1975 & 264 & 8.98 & 2.68 & 9.48 & 1.98 & 87 \\
\hline $909370 \%$ & 1976 & 259 & 10.55 & 2.78 & 10.23 & 2.36 & 87 \\
\hline 9093700 & 1977 & 321 & 11.70 & 2.89 & 11.64 & 1.69 & 96 \\
\hline 9093700 & 1978 & 224 & 9.78 & 2.88 & 11.67 & 1.91 & 92 \\
\hline 9093700 & 1979 & 90 & 8.87 & 2.48 & $a+10$ & 1.72 & 89 \\
\hline 9093700 & 1980 & 158 & 8.42 & 2.73 & 10.31 & 1.91 & 67 \\
\hline 9093700 & 1781 & 292 & 9.05 & 2.82 & 11.74 & 1.70 & 90 \\
\hline 9095500 & 1949 & 161 & 5.96 & 2.35 & 12.37 & 1.66 & 72 \\
\hline 9095500 & 1950 & 347 & 9.18 & 2.77 & 9.96 & 1.70 & 93 \\
\hline 9095500 & 1951 & 332 & 8.99 & 2.71 & 9.94 & 1.94 & 91 \\
\hline 9095500 & 1952 & 307 & 9.64 & 2.71 & 7.20 & 1.92 & 91 \\
\hline 9095500 & 1953 & 342 & 9.56 & 2.73 & 10.09 & 1.93 & 92 \\
\hline 9095500 & 1954 & 352 & 10.26 & 2.82 & 11.25 & 1.68 & 94 \\
\hline 9095500 & 1955 & 327 & 10.35 & 2.64 & 9.70 & 1.77 & 94 \\
\hline 9095500. & 1956 & 356 & 9.54 & 2.76 & 10.19 & 1.97 & 92 \\
\hline 9095500 & 1957 & 340 & 8.29 & 2.77 & 9.26 & 2.21 & 87 \\
\hline 9095500 & 1958 & 343 & 9.36 & 2.76 & 10.60 & 1.95 & 92 \\
\hline 9095500 & 1959 & 325 & 8.66 & 2.74 & 10.01 & 1.98 & 89 \\
\hline 9095500 & 1960 & 303 & 9.50 & 2.78 & 9.27 & 2.33 & 87 \\
\hline 9095500 & 1961 & 308 & 9.77 & 2.82 & 9.41 & 2.06 & 90 \\
\hline 9095500 & 1962 & 295 & 8.44 & 2.72 & 8.01 & 2.16 & 85 \\
\hline 9095500 & 1963 & 308 & 10.29 & 2.76 & 9.80 & 1.57 & 94 \\
\hline 9095500 & 1964 & 261 & 10.30 & 2.61 & 8.84 & 1.78 & 71 \\
\hline 9095500 & 1965 & 322 & 7.90 & 2.74 & 8.62 & 1.93 & 88 \\
\hline 9095500 & 1966 & 232 & $10 \cdot 14$ & 2.76 & 9.72 & 1.73 & 92 \\
\hline 9095500 & 1967 & 301 & 8.60 & 2.70 & 9.54 & 2.00 & 87 \\
\hline 9095500 & 1968 & 303 & 8.41 & 2.75 & 8.99 & 1.81 & 89 \\
\hline 9095500 & 1969 & 304 & 9.34 & 2.74 & 9.60 & 2.08 & 90 \\
\hline 9095500 & 1970 & 324 & 8.66 & 2.78 & 8.91 & 2.17 & 87 \\
\hline 9095500 & 1971 & 299 & 8.70 & 2.76 & 8.36 & 1.91 & 51 \\
\hline 8095500 & 1972 & 303 & 9.27 & 2.79 & 9.66 & 2.15 & 85 \\
\hline 9075500 & 1773 & 251 & 7.28 & 2.67 & 9.18 & 1.99 & 81 \\
\hline 9095500 & 1974 & 275 & 0.44 & 2.72 & 9.24 & 2.01 & 34 \\
\hline 9075500 & 1975 & 307 & 7.81 & 2.62 & 9.10 & 1.33 & 71 \\
\hline 9095500 & 1976 & 365 & 8.37 & 2.76 & 9.57 & 1.55 & 93 \\
\hline
\end{tabular}


Table 3.--Harmonic-function coefficients, Colorado River subbasin--Continued

\begin{tabular}{|c|c|c|c|c|c|c|c|}
\hline \multirow[b]{2}{*}{ Station no. ${ }^{2}$} & \multirow[b]{2}{*}{ Year } & \multirow[b]{2}{*}{ NO } & \multicolumn{5}{|c|}{ Harmonic coefficients ${ }^{1}$} \\
\hline & & & A & $\mathrm{C}$ & M & SE & PVAR \\
\hline $9095=00$ & 1977 & 320 & 10.22 & 2.91 & 9.99 & $1 \cdot 13$ & 97 \\
\hline 9095500 & 1978 & 341 & 7.24 & 2.85 & 9.19 & 2.04 & 85 \\
\hline 9095500 & 1979 & 254 & 7.68 & 2.77 & 0.91 & 1.65 & 88 \\
\hline 9095500 & 1980 & 130 & 11.19 & 2.47 & 7.72 & 1.64 & 99 \\
\hline 9095500 & 1981 & 365 & 8.64 & 2,81 & $11 \cdot 33$ & 1,56 & 93 \\
\hline 9152500 & 1949 & 149 & 7.84 & $2+24$ & 14.24 & 1.81 & 81 \\
\hline 7152500 & 1950 & 311 & 10.37 & 2.71 & 11.95 & 2.05 & 92 \\
\hline 9152500 & 1951 & 318 & 9.83 & 2.73 & $12+16$ & 2.14 & 91 \\
\hline 9152500 & 1952 & 317 & 9.33 & $2.7 \div$ & 12.15 & 2.10 & 91 \\
\hline 9152500 & 1953 & 330 & 11.05 & 2.71 & 12.50 & 2.94 & 87 \\
\hline 9152500 & 1954 & 328 & $11 \cdot 10$ & 2.90 & 13.78 & 2.41 & 91 \\
\hline 9152500 & 1955 & 335 & 11.58 & 2.67 & 11.53 & 2.08 & 93 \\
\hline 9152500. & $\begin{array}{l}1956 \\
1957\end{array}$ & $\begin{array}{l}353 \\
321\end{array}$ & $\begin{array}{r}10.34 \\
9.02\end{array}$ & $\begin{array}{l}2.80 \\
2.72\end{array}$ & $\begin{array}{l}12.62 \\
10,72\end{array}$ & $\begin{array}{l}2.47 \\
2.87\end{array}$ & $\begin{array}{l}89 \\
81\end{array}$ \\
\hline $\begin{array}{l}9152500 \\
9152500\end{array}$ & $\begin{array}{l}145 \% \\
1958\end{array}$ & $\begin{array}{l}321 \\
351\end{array}$ & $\begin{array}{r}9.02 \\
12.06\end{array}$ & $\begin{array}{l}2.72 \\
2.76\end{array}$ & 10.75 & $\begin{array}{l}2.87 \\
2.83\end{array}$ & $\begin{array}{l}81 \\
90\end{array}$ \\
\hline 9152500 & 1959 & 349 & 11.59 & 2.81 & 13.75 & 2.40 & 92 \\
\hline 9152500 & 1960 & 292 & 10.49 & 2.77 & 10.39 & 2.04 & 91 \\
\hline 9152500 & 1961 & 254 & 10.64 & 2.77 & 10.09 & 2.18 & 88 \\
\hline 9152500 & 19.62 & 264 & 9.89 & 2.72 & 9.16 & 2.10 & 87 \\
\hline 9152500 & 1963 & 283 & 10.22 & 2.74 & 10.83 & 1.64 & 94 \\
\hline 9152500 & 1964 & 281 & 9.76 & 2.61 & 10.19 & 1.75 & 52 \\
\hline 9152500 & 1965 & 298 & 8.23 & 2.68 & 9.17 & 2.01 & 86 \\
\hline 9152500 & 1966 & 289 & 9.95 & 2.75 & 10.77 & 1.82 & 91 \\
\hline 9152500 & 1967 & 318 & 9.41 & 2.72 & 10.82 & 2.05 & 90 \\
\hline 9152500 & 1968 & 348 & 7.75 & 2.74 & 9.99 & 2.09 & 86 \\
\hline 9152500 & 1969 & 340 & 9.97 & 2.77 & 10.23 & 1.93 & 92 \\
\hline 9152500 & 1970 & 354 & 8.06 & 2.73 & 9.74 & 2.05 & 88 \\
\hline 9152500 & 1972 & 366 & 9.81 & 2.86 & $11 \cdot 61$ & 1.71 & 93 \\
\hline 9152500 & 1973 & 365 & 9.52 & 2.80 & 11,71 & 2.23 & 89 \\
\hline 9152500 & 1974 & 297 & 9.91 & 2.71 & 12.06 & $1 . \overline{81}$ & 90 \\
\hline 9152500 & 1976 & 327 & 10.05 & 2.79 & $11.7 \dot{6}$ & 1.63 & 95 \\
\hline 9152500 & 1977 & 271 & 11.16 & 2.95 & 11.69 & 1.91 & 91 \\
\hline 9152500 & 1978 & 305 & 9.05 & 2.77 & 10.76 & 2.51 & 85 \\
\hline 9152500 & 1979 & 176 & 8.77 & 2.65 & 10.98 & 2.02 & 83 \\
\hline 9152500 & 1980 & 173 & 8.64 & 2.45 & 11.32 & 1.95 & 87 \\
\hline 9152500 & 1981 & 365 & 9.25 & 2.80 & 12.69 & 1.56 & 94 \\
\hline 9163530. & 1962 & 143 & 11.11 & 2.46 & 10.98 & .79 & 91 \\
\hline 9163530 & 1965 & 358 & 9.34 & 2.78 & 10.44 & 2.15 & 50 \\
\hline 9163530. & 1966 & 315 & 11.12 & 2.81 & 12.17 & 1.82 & 94 \\
\hline
\end{tabular}


Table 3.--Harmonic-function coefficients, Colorado River subbasin--Continued

\begin{tabular}{|c|c|c|c|c|c|c|c|}
\hline \multirow[b]{2}{*}{ Station no. ${ }^{2}$} & \multicolumn{7}{|c|}{ Harmonic coefficients ${ }^{1}$} \\
\hline & Year & NO & A & C & M & $\mathrm{SE}$ & PVAR \\
\hline 9163530. & 1967 & 320 & 9.96 & 2.79 & 11.82 & 2.09 & 91 \\
\hline 9163530 & 1968 & 262 & 10.26 & 2.84 & 11.00 & 1.91 & 92 \\
\hline 9163530 & 1969 & 173 & 8.37 & 2.91 & 11.70 & 2.31 & 81 \\
\hline 9163530 & 1974 & 339 & 10,54 & 2.71 & 11.31 & 1.84 & 93 \\
\hline 9163530 & 1975 & 273 & 7.87 & 2.56 & 12.42 & 2.03 & 83 \\
\hline 9163530 & 1976 & 360 & 10.23 & 2.82 & 11.91 & 1.65 & 95 \\
\hline 9163530 & 1977 & 357 & 11.47 & 2.91 & 11.59 & 1.40 & 97 \\
\hline 9163530 & 1978 & 343 & 8.41 & 2.75 & 11.00 & 2.09 & 88 \\
\hline 9163530 & 1979 & 341 & 9.75 & 2.69 & 10.42 & 2.01 & 92 \\
\hline 9163530 & 1980 & 309 & 10.26 & 2.78 & 12.47 & 2.39 & 99 \\
\hline 9163530 & 1981 & 338 & 9.53 & 2.95 & 12.94 & 1.86 & 93 \\
\hline 9180000. & 1950 & 256 & 10.26 & 2.75 & 11.33 & 1.98 & 99 \\
\hline 9180000 & 1951 & 179 & 8.70 & 2.79 & 13.76 & 2.13 & 99 \\
\hline 9180000 & 1952 & 238 & 10.10 & 2.70 & 11.04 & 1.87 & 99 \\
\hline 9180000. & $195 \overline{3}$ & 200 & 11.73 & 2.68 & 12.12 & 1.97 & 99 \\
\hline 9180000 & 1954 & 224 & 11.09 & 2.87 & $12 \cdot 40$ & 1.80 & 99 \\
\hline 9180000 & 1955 & 232 & 12.54 & 2.69 & 11.50 & 1.87 & 99 \\
\hline 9180000 & 1956 & 228 & 11.27 & 2.77 & 12.20 & 2.08 & 99 \\
\hline 9180000 & 1957 & 197 & 9.86 & 2.68 & 10.34 & 2.30 & 99 \\
\hline 9180000 & 1958 & 182 & 11.42 & 2.67 & 11.09 & 1.92 & 99 \\
\hline 9180000 . & 1959 & 170 & 11.75 & 2.84 & 12.17 & 2.74 & 99 \\
\hline 9180000. & 1965 & 222 & 10.04 & 2.75 & $11 \cdot 24$ & 2.47 & 98 \\
\hline 9180000 & 1966 & 204 & 12.39 & 2.80 & 12.80 & 2.43 & 95 \\
\hline 9180000 & 1967 & 221 & 11.67 & 2.84 & 12.90 & 2.59 & 80 \\
\hline 9180000 & 1969 & 265 & 11.60 & 2.75 & 12.31 & 2.38 & 93 \\
\hline 9180000 & 1970 & 268 & 10.37 & 2.81 & 11.39 & 2.40 & 90 \\
\hline 9180000 . & 1971 & 280 & 9.56 & 2.82 & 11.26 & 2.08 & 93 \\
\hline 9180000 & 1972 & 248 & $11 \cdot 14$ & 2.93 & 13.59 & 2.13 & 95 \\
\hline 9180000. & $197 \overline{3}$ & 230 & 11.13 & 2.64 & $10+6=$ & 2.57 & 90 \\
\hline 9180000 & 1974 & 240 & 11.51 & 2.82 & 12.16 & 1.98 & 99 \\
\hline 7180000 & 1975 & 270 & 9.66 & 2.68 & 11.47 & 2.59 & 90 \\
\hline 9180000 & 1976 & 293 & 11.14 & 2.93 & 11.97 & 1.93 & 94 \\
\hline 9180000 & 1977 & 312 & 13.61 & 2.92 & 12.40 & 2.09 & 94 \\
\hline 9100000 & 1978 & 291 & 11.12 & 2.67 & 11.78 & 2.45 & 97 \\
\hline 9180000 & 1979 & 289 & 11.53 & 2.58 & 11.51 & 2.38 & 99 \\
\hline 9180000 & 1980 & 285 & 9.70 & 2.63 & 12.08 & 2.90 & 87 \\
\hline 9180000 & 1781 & 301 & $11 \cdot 17$ & 2.81 & 14.13 & 1.77 & 92 \\
\hline 9180500 & 1950 & 260 & 10.34 & 2.75 & 11.37 & 1.94 & 99 \\
\hline 9180500. & 1951 & 280 & 9.74 & 2.77 & 11.68 & 2.04 & 96 \\
\hline
\end{tabular}


Table 3.--Harmonic-function coefficients, Colorado River subbasin--Continued

\begin{tabular}{|c|c|c|c|c|c|c|c|}
\hline \multirow[b]{2}{*}{ Station no. ${ }^{2}$} & \multicolumn{7}{|c|}{ Harmonic coefficients ${ }^{1}$} \\
\hline & Year & NO & A & $\mathrm{C}$ & M & $\mathrm{SE}$ & PVAR \\
\hline 9180500 & 1952 & 223 & 10.32 & 2.72 & 10.74 & 1.89 & 99 \\
\hline 9180500 & 1953 & 154 & 11.75 & 2.70 & 12.20 & 2.06 & 99 \\
\hline 9180500 & 1954 & 256 & 10.77 & 1.82 & 12.51 & 1.82 & 99 \\
\hline 9180500 & 1955 & 249 & 11.64 & 2.68 & 10.76 & 1.87 & 99 \\
\hline 9180500 & 1956 & 253 & 10.37 & 2.79 & 11.08 & 1.96 & 99 \\
\hline 9180500 & 1957 & 245 & 9.74 & 2.69 & 9.89 & 2.38 & 99 \\
\hline 9180500 & 1758 & 207 & 10.94 & 2.70 & 11.15 & 2.14 & 99 \\
\hline 9180500 & 1959 & 211 & 11.33 & 2.81 & 11.62 & 1.81 & 99 \\
\hline 9180500. & 1960 & 208 & 11.03 & 2.74 & 11.63 & $2 \cdot 12$ & 99 \\
\hline 9180500 & 1961 & 166 & 11.59 & 2.78 & 11.16 & 2.01 & 99 \\
\hline 9180500 & 1962 & 150 & 10.38 & 2.74 & 11.70 & 2.19 & 99 \\
\hline 9180500. & 1763 & 139 & 11.25 & 2.78 & 13.02 & 1.96 & 99 \\
\hline 9180500 & 1964 & 155 & 11.30 & 2.67 & 11.73 & 2.14 & 95 \\
\hline 9180500 & 1965 & 232 & 9.49 & 2.78 & 10.40 & 2.31 & 98 \\
\hline 9180500 & 1966 & 250 & $12 \cdot 32$ & 2.79 & 12.60 & 2.05 & 92 \\
\hline 9180500 & 1967 & 220 & 10.92 & 2.73 & 12.78 & 2.59 & 95 \\
\hline 9180500 & 1968 & 309 & 11.13 & 2.70 & 11.76 & 2.48 & 90 \\
\hline 9180500. & 1967 & 298 & 11.23 & 2.72 & 11.76 & 2.37 & 90 \\
\hline 9180500 & 1970 & 291 & 9.44 & 2.77 & 10.79 & 2.44 & 86 \\
\hline 9180500 & 1971 & 294 & 8.60 & 2.79 & 10.38 & 1.88 & 91 \\
\hline 9180500 & 1972 & 281 & 10.22 & 2.90 & 12.16 & 2.20 & 89 \\
\hline 9180500 & 1973 & 289 & 10.44 & 2.68 & 10.64 & 2.41 & 99 \\
\hline 9180500 & 1974 & 345 & 10.15 & 2.79 & 11.22 & 1.84 & 94 \\
\hline 9180500 & 1975 & 342 & 9.81 & 2.70 & 11.14 & 2.20 & 91 \\
\hline 9180500 & 1976 & 352 & 10.24 & 2.87 & 11.57 & 1.91 & 92 \\
\hline 5180500 & 1977 & 338 & 12.60 & 2.90 & 11.61 & 1.82 & 99 \\
\hline 9180500 & 1978 & 355 & 9.17 & 2.70 & 12.17 & 2.22 & 89 \\
\hline 9180500 & 1979 & 316 & 11.06 & 2.62 & 11.15 & 2.18 & 99 \\
\hline 9180500. & 1980 & 154 & 13.25 & 2.69 & 11.12 & 1.55 & 99 \\
\hline 9180500 & 1981 & 350 & 10.58 & 2.82 & 13.61 & 1.72 & 94 \\
\hline
\end{tabular}

${ }^{1}$ See equation (1), p. 2 of text.

${ }^{2}$ See supplementary information for station description. 

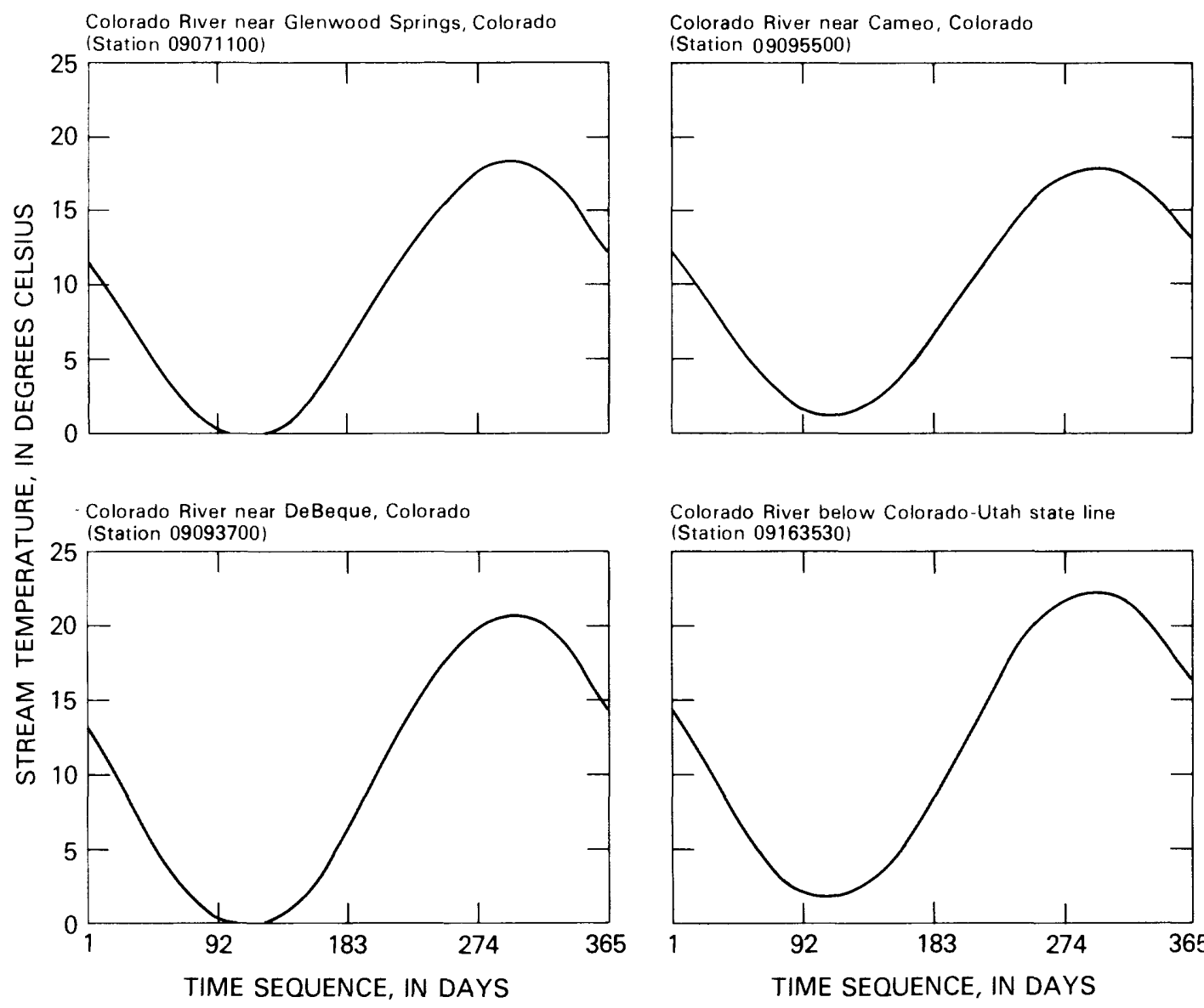

Colorado River below Colorado-Utah state line (Station 09163530)

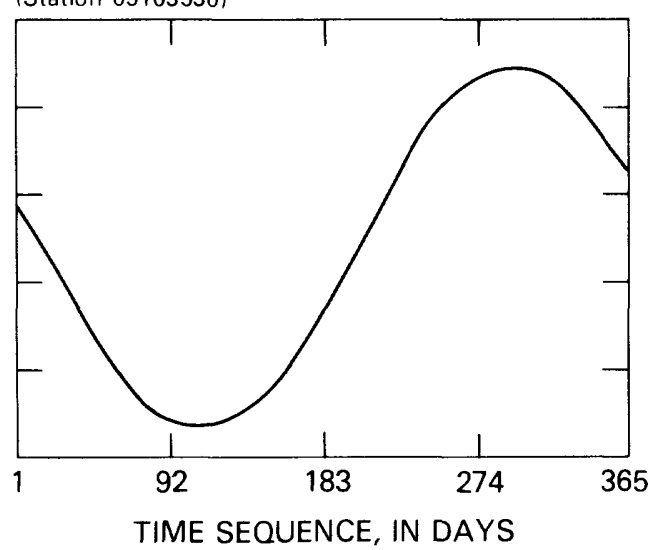

Figure 3.--Stream-reach seasonal water-temperature patterns, Colorado River, 1976 water year. 
Table 4.--Harmonic-function coefficients, San Juan River subbasin

[NO = number of daily values. $\mathrm{SE}=$ standard error or estimate. PVAR = percent variance explained by the harmonic function.]

\begin{tabular}{|c|c|c|c|c|c|c|c|}
\hline \multirow[b]{2}{*}{ Station no. ${ }^{2}$} & \multicolumn{7}{|c|}{ Harmonic coefficients ${ }^{1}$} \\
\hline & Year & NO & $\mathrm{A}$ & $\mathrm{C}$ & M & $\mathrm{SE}$ & PVAR \\
\hline 9355500 & 1955 & 215 & 9.29 & 2.58 & 11.10 & 2.69 & 81 \\
\hline 9355500 & 1956 & 324 & 9.96 & 2.79 & 11.08 & 2.27 & 90 \\
\hline 9355500 & 1957 & 285 & 7.56 & 2.65 & 9.22 & 2.30 & $B 1$ \\
\hline 9355500 & 1955 & 358 & 9.72 & 2.66 & 9.26 & 1.76 & 93 \\
\hline 9355500 & 1959 & 355 & 11.06 & 2.92 & 10.55 & 1.56 & 95 \\
\hline 9355500 & 1960 & 269 & $11 \cdot 98$ & 2.64 & 9.08 & 2.89 & 83 \\
\hline 9355500 & 1961 & 353 & 11.13 & 2.79 & 11.67 & 2.09 & 93 \\
\hline 9355500 & 1962 & 303 & 11.51 & 2.72 & 11.36 & 2.42 & 89 \\
\hline 9355500 & 1963 & 337 & 7.63 & 2.06 & 11.91 & 2.25 & 85 \\
\hline 9355500 & 1964 & 347 & 6.19 & 2.34 & 10.22 & 2.01 & 83 \\
\hline 9355500 & 1765 & 316 & 5.08 & 2.35 & 7.85 & 1.95 & 75 \\
\hline 9355500 & 1966 & 352 & 5.53 & 2.24 & 10.02 & 2.26 & 75 \\
\hline 9355500 & 1767 & 364 & 4.47 & 2.61 & 9.06 & 2.84 & 55 \\
\hline 9355500 & 1968 & 359 & 5.40 & 2.44 & 9.26 & 2.31 & 73 \\
\hline 9355500 & 1969 & 101 & 4.75 & 2.21 & 7.82 & 1.64 & 65 \\
\hline 9357000 & 1959 & 333 & 10.21 & 2.81 & 14.08 & 6.23 & 56 \\
\hline 9357000 & 1960 & 324 & 11.17 & 2.78 & 12.49 & 2.53 & 89 \\
\hline 9357000 & 1761 & 340 & 11.02 & 2.83 & 12.94 & 2.53 & 89 \\
\hline 9357000 & 1962 & 336 & 11.27 & 2.77 & 12.58 & 2.64 & 89 \\
\hline 9357000 & 1963 & 333 & 11.16 & 2.82 & 15.22 & 2.37 & 90 \\
\hline 9364500 & 1959 & 357 & 9.51 & 2.82 & 14.43 & 5.34 & 60 \\
\hline 9364500 & 1960 & 350 & 10.20 & 2.74 & 12.46 & 3.92 & 76 \\
\hline 9364500 & 1961 & 363 & 9.96 & 2.79 & 12.80 & 3.01 & 8.4 \\
\hline 9364500 & 1962 & 345 & 10.06 & 2.73 & 12.75 & 3.26 & 82 \\
\hline 9364500 & 1963 & 348 & 10.54 & 2.77 & 13.66 & 2.48 & 89 \\
\hline 9364500. & 1764 & 346 & 10.74 & 2.72 & 13.37 & 2.63 & 89 \\
\hline 7364500 . & 1965 & 347 & 7.76 & 2.76 & 12.67 & 2.95 & 77 \\
\hline 9564500 & 1966 & 344 & 10.64 & 2.71 & 13.43 & 2.60 & 89 \\
\hline 9364500 & 1767 & 350 & 9.99 & 2.77 & 14.46 & 2.83 & 86 \\
\hline 9364500 & 1968 & 309 & 7.42 & 2.54 & 12.77 & 3.77 & 64 \\
\hline 9364500 & 1969 & 352 & 9.32 & 2.57 & 11.86 & 2.77 & 84 \\
\hline 9364500 & 1970 & 355 & 9.23 & 2.88 & 12.51 & 3.05 & 82 \\
\hline 9364500 & 1971 & 335 & 9.27 & 2.80 & $12 \cdot 83$ & 3.20 & 80 \\
\hline 9364500 & 1972 & 537 & 10.10 & 2.85 & $13 \cdot 13$ & 3.20 & 82 \\
\hline 9364500 & 1973 & 331 & 8.79 & 2.61 & 10.09 & 3.01 & 80 \\
\hline 7364500 & 1774 & 363 & 11.12 & 2.73 & 13.75 & 3.09 & 86 \\
\hline 9364500 & $197 \vdots$ & 332 & 8.36 & 2.48 & 10.77 & 2.99 & 79 \\
\hline 9364500 & 1976 & 361 & 9.03 & 2.89 & 12.56 & 2.27 & 88 \\
\hline 7364500. & 1977 & 254 & 10.97 & 2.95 & 13.97 & 3.12 & 85 \\
\hline
\end{tabular}


Table 4.--Harmonic function coefficients, San Juan River subbasin--Continued

\begin{tabular}{|c|c|c|c|c|c|c|c|}
\hline \multirow[b]{2}{*}{ Station no. ${ }^{2}$} & \multirow[b]{2}{*}{ Year } & \multicolumn{4}{|c|}{ Harmonic coefficients ${ }^{1}$} & \multirow[b]{2}{*}{$\mathrm{SE}$} & \multirow[b]{2}{*}{ PVAR } \\
\hline & & NO & A & $\mathrm{C}$ & M & & \\
\hline $\begin{array}{l}9364500 \\
9364500 \\
9364500 \\
9364500\end{array}$ & $\begin{array}{l}1979 \\
1979 \\
1980 \\
1981\end{array}$ & $\begin{array}{l}349 \\
277 \\
365 \\
360\end{array}$ & $\begin{array}{r}8.68 \\
8.56 \\
10.16 \\
10.38\end{array}$ & $\begin{array}{l}2.65 \\
2.45 \\
2.65 \\
2.71\end{array}$ & $\begin{array}{l}11.59 \\
12.53 \\
14.23 \\
12.89\end{array}$ & $\begin{array}{l}3.35 \\
2.97 \\
2.68 \\
2.68\end{array}$ & $\begin{array}{l}77 \\
68 \\
85 \\
86\end{array}$ \\
\hline $\begin{array}{l}9365000 . \\
9365000 . \\
9365000 \\
9365000 . \\
9365000 . \\
9365000 . \\
9365000 . \\
9355000 . \\
9365000 . \\
9365000 . \\
9365000 . \\
9365000 . \\
9365000 . \\
9365000 . \\
9365000 . \\
9365000 . \\
9365000 . \\
9365000 .\end{array}$ & $\begin{array}{l}1964 \\
1965 \\
1966 \\
1967 \\
1768 \\
1969 \\
1970 \\
1971 \\
1972 \\
1973 \\
1974 \\
1975 \\
1976 \\
1977 \\
1978 \\
1979 \\
1980 \\
1981\end{array}$ & $\begin{array}{l}351 \\
350 \\
327 \\
349 \\
315 \\
264 \\
354 \\
340 \\
355 \\
352 \\
363 \\
337 \\
354 \\
283 \\
350 \\
278 \\
365 \\
329\end{array}$ & $\begin{array}{r}9.70 \\
7.34 \\
10.23 \\
9.77 \\
7.67 \\
8.24 \\
9.14 \\
8.67 \\
9.03 \\
6.16 \\
9.03 \\
6.93 \\
8.91 \\
9.87 \\
8.08 \\
6.51 \\
8.20 \\
8.33\end{array}$ & $\begin{array}{l}2.73 \\
2.69 \\
2.69 \\
2.81 \\
2.60 \\
2.48 \\
2.84 \\
2.83 \\
2.91 \\
2.70 \\
2.82 \\
2.59 \\
2.95 \\
2.93 \\
2.78 \\
2.53 \\
2.72 \\
2.83\end{array}$ & $\begin{array}{r}13.59 \\
12.28 \\
14.22 \\
14.20 \\
13.18 \\
13.46 \\
12.54 \\
13.19 \\
13.12 \\
8.70 \\
13.38 \\
9.54 \\
12.04 \\
12.47 \\
11.49 \\
10.90 \\
12.62 \\
12.02\end{array}$ & $\begin{array}{l}2.48 \\
2.92 \\
2.02 \\
2.95 \\
3.41 \\
2.46 \\
3.17 \\
3.09 \\
2.97 \\
2.02 \\
2.21 \\
2.03 \\
2.09 \\
2.55 \\
3.08 \\
2.47 \\
1.99 \\
2.21\end{array}$ & $\begin{array}{l}88 \\
76 \\
92 \\
84 \\
68 \\
87 \\
80 \\
78 \\
81 \\
82 \\
87 \\
85 \\
70 \\
89 \\
77 \\
66 \\
87 \\
88\end{array}$ \\
\hline $\begin{array}{l}9368000 . \\
9368000 . \\
9368000 . \\
9368000 . \\
9368000 . \\
9368000 . \\
9368000 . \\
9368000 . \\
9368000 . \\
9368000 . \\
9368000 . \\
9368000 . \\
9368000 . \\
9368000 \\
9368000 . \\
9368000 . \\
9368000 .\end{array}$ & $\begin{array}{l}1959 \\
1960 \\
1961 \\
1962 \\
1963 \\
1964 \\
1965 \\
1966 \\
1967 \\
1968 \\
1969 \\
1970 \\
1971 \\
1972 \\
1973 \\
1574 \\
1775\end{array}$ & $\begin{array}{l}337 \\
307 \\
333 \\
357 \\
357 \\
362 \\
364 \\
333 \\
363 \\
338 \\
354 \\
358 \\
360 \\
355 \\
351 \\
358 \\
335\end{array}$ & $\begin{array}{r}9.60 \\
10.76 \\
11.55 \\
11.66 \\
12.01 \\
11.21 \\
8.81 \\
10.41 \\
9.19 \\
9.95 \\
8.68 \\
8.36 \\
7.26 \\
8.27 \\
7.87 \\
10.92 \\
9.29\end{array}$ & $\begin{array}{l}2.85 \\
2.76 \\
2.88 \\
2.73 \\
2.75 \\
2.73 \\
2.76 \\
2.75 \\
2.91 \\
3.02 \\
2.73 \\
2.90 \\
2.79 \\
3.01 \\
2.62 \\
2.85 \\
2.63\end{array}$ & $\begin{array}{l}13.95 \\
12.78 \\
14.10 \\
13.13 \\
14.97 \\
13.95 \\
12.91 \\
14.20 \\
14.43 \\
13.60 \\
11.73 \\
12.58 \\
11.76 \\
12.58 \\
9.70 \\
13.97 \\
10.70\end{array}$ & $\begin{array}{l}7.00 \\
2.49 \\
2.75 \\
2.63 \\
2.21 \\
2.30 \\
2.95 \\
2.29 \\
2.79 \\
3.35 \\
2.64 \\
3.15 \\
2.44 \\
3.17 \\
2.41 \\
1.86 \\
1.72\end{array}$ & $\begin{array}{l}48 \\
88 \\
89 \\
90 \\
93 \\
92 \\
92 \\
90 \\
84 \\
80 \\
84 \\
77 \\
78 \\
77 \\
84 \\
94 \\
89\end{array}$ \\
\hline
\end{tabular}


Table 4.--Harmonic function coefficients, San Juan River subbasin--Continued

\begin{tabular}{|c|c|c|c|c|c|c|c|}
\hline \multirow[b]{2}{*}{ Station no. ${ }^{2}$} & \multirow[b]{2}{*}{ Year } & \multicolumn{4}{|c|}{ Harmonic coefficients ${ }^{1}$} & \multirow[b]{2}{*}{ SE } & \multirow[b]{2}{*}{ PVAR } \\
\hline & & NO & A & $\mathrm{C}$ & M & & \\
\hline 726300 & 1070 & 353 & 7.35 & $=.33$ & $\therefore 2.49$ & $\pm .5=$ & 95 \\
\hline $73 \leq 50 \%$ & \pm 577 & $3: 5$ & $9+90$ & 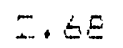 & $1=53$ & $2.2 \Delta$ & 34 \\
\hline $7=0800$ & 1973 & $\div 4$ & $1 \equiv .07$ & $\because .99$ & 16.25 & 2.5 & $7-$ \\
\hline $73 \leq 50$ & 1779 & בְ & 7.3 & $= \pm$ & G. E4 & $1.7 \equiv$ & $5^{-}$ \\
\hline $7503 \%$ & 1980 & 397 & 7.90 & $\therefore .52$ & $11 .=$ & $2 \cdot 1 \pm$ & $\Xi \Xi$ \\
\hline $9305 \div$ & $199:$ & 305 & 8.57 & 2.52 & $12+44$ & $\perp \cdot \bar{E}$ & $\overline{7}:$ \\
\hline $9 \Xi 7950$ & 1745 & $3+3$ & $11, \Xi \because$ & 2.76 & $12= \pm=$ & $\Sigma . \bar{Z}$ & 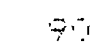 \\
\hline $9=7950 \%$ & 1545 & $\Xi 20$ & $12 . \pm 4$ & $2 . E 5$ & $-3+\ldots$ & $\therefore E$ & $a \Xi$ \\
\hline 337550 & はマ4 & $\equiv 4 \equiv$ & 10,97 & 2. & $12.5=$ & $\therefore 23$ & $\overline{72}$ \\
\hline $737=50$ & 1945 & 341 & $11, \pm 0$ & 2.72 & $\therefore+60$ & 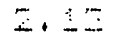 & ラב \\
\hline $7 \Xi 7=\Xi 0 \%$ & 1949 & $\Xi \Xi 2$ & $1 \pm \cdot 6$ & $\therefore 5$ & $\therefore 1,7=$ & $2 .+5$ & 83 \\
\hline $73-7500$ & \pm 750 & 360 & 11.75 & $\Xi .9 E$ & $14.6=$ & 5.5 & $9=$ \\
\hline 757750 & $\$ 951$ & 259 & 5.79 & 2.87 & 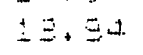 & $=05$ & 91 \\
\hline 537500 & 1952 & 277 & 10.77 & $\equiv 72$ & $\pm=E$ & $\therefore 0 \%$ & 81 \\
\hline $9579500+$ & $1=53$ & 355 & 10.4 & $2.3 \pm$ & $1 \pm .27$ & $=30$ & $8 \overline{7}$ \\
\hline $737950 \%$ & 1954 & $\Xi \Xi t$ & 11.11 & Z.E & $1=.53$ & $2+20$ & Eit \\
\hline 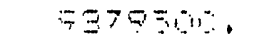 & $17=5$ & 300 & 16,77 & 2.79 & $1 \ldots,=$ & $\therefore \div$ & $7 a$ \\
\hline $93753 \%$ & 1956 & $34=$ & 10,0 & $2 . E 5$ & $\therefore \Sigma+\Xi$ & 2.5 & $=0$ \\
\hline 9877560 & $\therefore 737$ & $\equiv 19$ & $10+12$ & $\Xi . E 5$ & $1 \because+1$ & $\Xi E=$ & 34 \\
\hline 957550 & 1955 & 300 & $\therefore 1,45$ & $=30$ & $1=.34$ & 2.95 & 73 \\
\hline Уラ7テ500. & 1759 & $3: 44$ & 10,41 & 2.57 & $12 \cdot E 7$ & 2.31 & 7 \\
\hline 9579500 & 196 & $3=9$ & $\pm 1+4$ & 2.32 & $\pm 2.5 \pm$ & 2.67 & 55 \\
\hline 9379500 & $10 \dot{1}$ & $\because 45$ & $11 .=7$ & $\Sigma .5 \overline{5}$ & $\pm 2,15$ & $1.7 \equiv$ & 75 \\
\hline 957500 & $\therefore 962$ & \pm 16 & $11: 4 \%$ & 2.57 & $12.3=$ & $2.4 \Xi$ & $\overline{7}$ \\
\hline 757950 & $\pm \leq 3$ & 150 & 11,37 & $=.77$ & 15,33 & $2 \cdot-2$ & 99 \\
\hline 7375500 & 1764 & $\therefore 24$ & \pm \pm .05 & 2.35 & $\pm \equiv, 5$ & 2.7 & $=1$ \\
\hline 9379500 & 1953 & 295 & $7 .=2$ & 2.92 & 12.20 & $=.33$ & $E 1$ \\
\hline 937950 & $1=6$ & 3t: & $11 . \varepsilon e$ & $2.7 \%$ & $14+5$ & $I+E$ & $\overline{75}$ \\
\hline 9377500 & 1957 & Ect & \pm 1.30 & 2.36 & $\pm \overline{3} \cdot \overrightarrow{37}$ & 1.73 & $=4$ \\
\hline 5577500 & 1960 & 304 & $\pm 1.5=$ & $2 . \pm 8$ & 12.75 & $=5$ & 70 \\
\hline $757950 \%$ & $\therefore 5 \div$ & $3 \dot{0}+$ & $1=6$ & $\therefore .3$ & 13.47 & $1,=$ & 91 \\
\hline 737750. & $\therefore 770$ & $=4$ & $\pm 1+5$ & 2.34 & 15.34 & 2.5 & $\%$ \\
\hline 9577500 & $\pm 77 \pm$ & $E: 4$ & $1 \therefore 93$ & 2.94 & $\therefore-7 \%$ & 25 & \pm 5 \\
\hline 937900 & $\therefore 7-2$ & $\Xi: 3$ & $\therefore 2+47$ & 2.55 & $1 \pm+27$ & 2.27 & 72 \\
\hline $0 E 7=50$, & 1773 & 203 & 11.45 & 2.5 & $1=2=$ & $1 .,:=$ & 97 \\
\hline $5=795 \%$ & $\therefore 77$ & $29=$ & 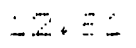 & $\therefore 74$ & $\therefore=\because 7$ & $\therefore=7$ & $\overline{7}$ \\
\hline 987950 & $\therefore 7$ & $\Xi=$ & $\therefore \therefore \equiv$ & $\therefore, \cdots$ & $\pm-\Sigma^{-}$ & & 78 \\
\hline $95^{-0=0}$ & $\pm \because 7=$ & 294 & $19+\infty$ & $3.0=$ & 13.17 & $\therefore 20$ & 92 \\
\hline $797010+$ & $\therefore \because \div$ & 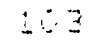 & 11.74 & 2. 54 & $12+5=$ & $=.34$ & 00 \\
\hline 9379569 & $\therefore \mp 7 \%$ & 31 & 11.70 & $2.5 \overline{7}$ & $11 \cdot=3$ & 1.27 & 99 \\
\hline $93795 \%$ & $15 \equiv I$ & $=3$ & $21+20$ & $2 .=i$ & 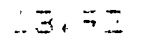 & $1 . E E$ & 59 \\
\hline
\end{tabular}

${ }^{1}$ See equation (1), p. 2 of text.

${ }^{2}$ See supplementary information for station description. 


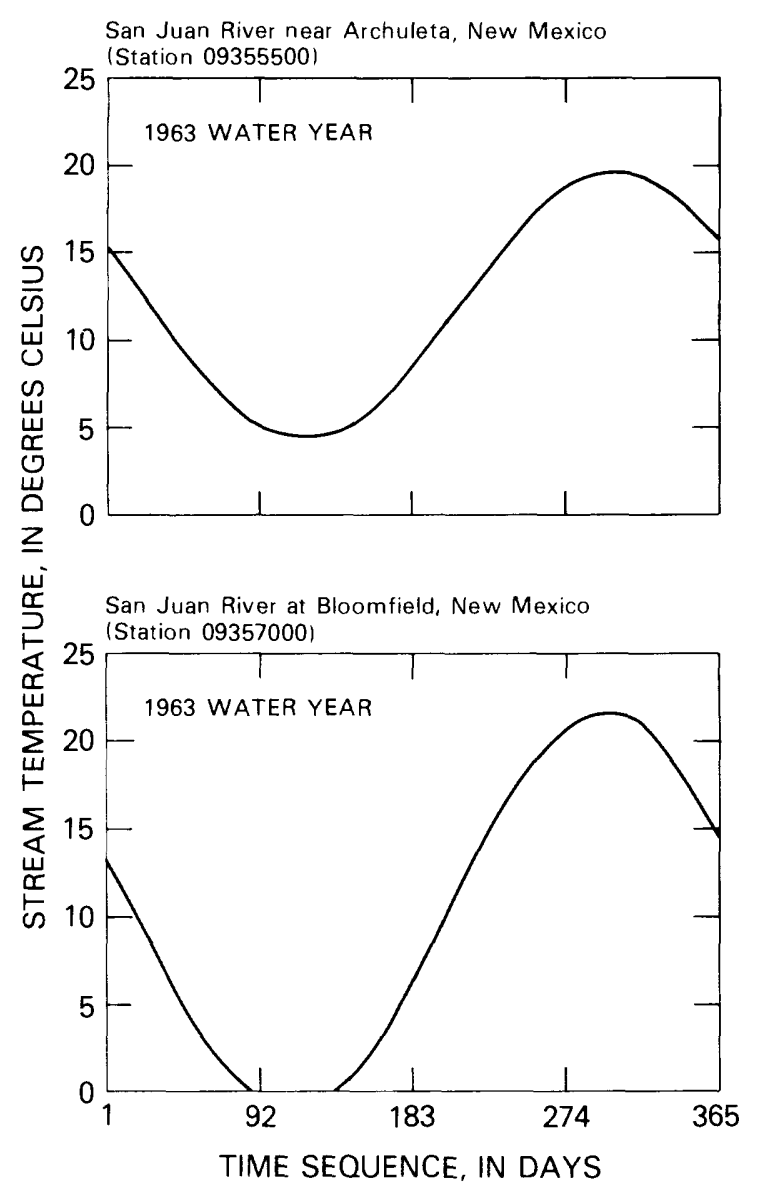

San Juan River at Farmington, New Mexico

(Station 09364500)

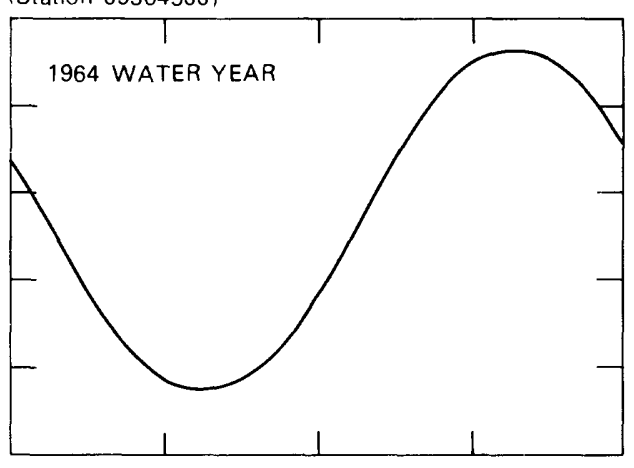

San Juan River at Shiprock, New Mexico (Station 09368000)

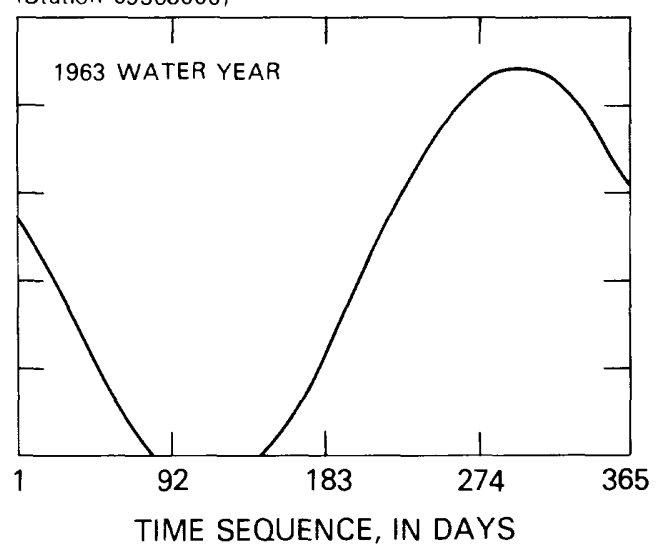

Figure 4.--Stream-reach seasonal water-temperature patterns, San Juan River, 1963 or 1964 water year. 
Animas River at Farmington, N. Mex., were quite similar to mean water temperatures at station 09365000 San Juan River at Farmington, N. Mex.; amplitude coefficients were consistently 1 to $2^{\circ} \mathrm{C}$ greater for the Animas River records.

\section{Green River Subbasin}

Daily water-temperature data for 11 sites in the Green River subbasin were analyzed in this study--8 were main-stem sites and 3 were on major tributaries (Duschesne, Price, and San Rafael Rivers in Utah) (see table 10 in the supplemental information section at the end of the report). A total of 226 station years of record was analyzed; harmonic-coefficient results are summarized in table 5 .

The Green River subbasin is the largest in the Upper Colorado River Basin system (Iorns and others, 1965). Lowham (1978) developed regional relationships for the harmonic mean and amplitude coefficients and compared estimates from these relationships with observed stream-reach averages for the Green River in Wyoming (see figure 8 of Lowham's 1978 report). Flaming Gorge Reservoir is located between station 09217000 Green River near Green River, Wyo., and station 09234500 Green River near Greendale, Utah. Reservoir operations began in 1963, and the impacts on downstream water temperature are clearly documented by the record at Greendale, Utah, and to a lesser extent further downstream at station 09261000 Green River near Jensen, Utah.

Using harmonic coefficients for the 1976 water year, upstream-downstream changes in the harmonic mean and amplitude coefficients for the six main-stem Green River stations with records for 1976 are summarized from table 5:

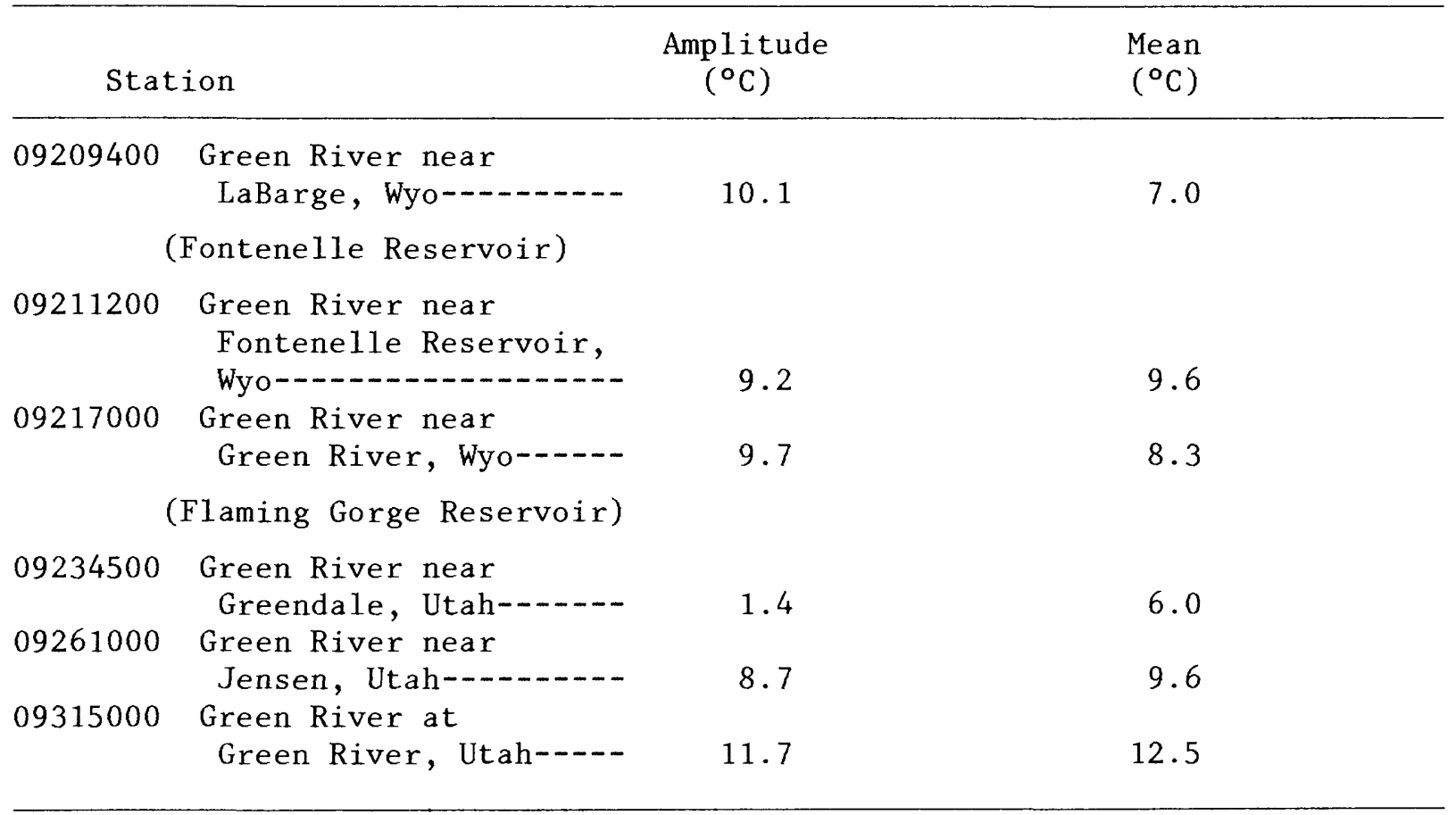


Table 5.--Harmonic-function coefficients, Green River subbasin

[NO = number of daily values; $\mathrm{SE}=$ standard error of estimate; PVAR = percent variance explained by the harmonic function]

\begin{tabular}{|c|c|c|c|c|c|c|c|}
\hline \multirow[b]{2}{*}{ Station no. ${ }^{2}$} & \multirow[b]{2}{*}{ Year } & \multicolumn{4}{|c|}{ Harmonic coefficients ${ }^{1}$} & \multirow[b]{2}{*}{$\mathrm{SE}$} & \multirow[b]{2}{*}{ PVAR } \\
\hline & & NO & $\mathrm{A}$ & $\mathrm{C}$ & M & & \\
\hline 9188500 & 1963 & 313 & 5.76 & 2.66 & 5.62 & 2.32 & 74 \\
\hline 9188500 & 1964 & 319 & 5.71 & 2.72 & 5.14 & 1.91 & 81 \\
\hline 9209400 & 1964 & 258 & 10.73 & 2.68 & 7.79 & 2.58 & 85 \\
\hline 9209400 & 1965 & 249 & 10.25 & 2.77 & 7.30 & 2.04 & 88 \\
\hline 9209400 & 1966 & 271 & 11.37 & 2.74 & 8.40 & 2.26 & 90 \\
\hline 9209400 & 1967 & 272 & 10.95 & 2.73 & 7.61 & 2.30 & 88 \\
\hline 9209400 & 1968 & 291 & 10.37 & 2.81 & 7.56 & 2.07 & 90 \\
\hline 9209400 & 1969 & 261 & 10.58 & 2.76 & 8.27 & 2.26 & 89 \\
\hline 9209400 & 1970 & 270 & 11.18 & 2.80 & 7.76 & 2.47 & 88 \\
\hline 9209400. & 1971 & 240 & 11.79 & 2.81 & 6.47 & 2.12 & $B B$ \\
\hline 9209400 & 1972 & 216 & 11.12 & 2.81 & 7.26 & 1.82 & 89 \\
\hline 9209400 & 1973 & 221 & 12.49 & 2.69 & 6.14 & 2.26 & 85 \\
\hline 9209400. & 1974 & 257 & 10.61 & 2.79 & 7.57 & 2.09 & 88 \\
\hline 9209400 & 1975 & 250 & 10.16 & 2.64 & 5.75 & 1.92 & 89 \\
\hline 9209400. & 1976 & 284 & 10.13 & 2.74 & 7.04 & 2.15 & 89 \\
\hline 9211200 & 1968 & 350 & 8.29 & 2.70 & 8.06 & 1.82 & 90 \\
\hline 9211200 & 1969 & 363 & 8.34 & 2.56 & 8.82 & 1.47 & 54 \\
\hline 9211200. & 1970 & 365 & 8.01 & 2.60 & 9.15 & 1.61 & 92 \\
\hline 9211200. & 1971 & 365 & 8.40 & 2.64 & 9.22 & 1.39 & 94 \\
\hline 9211200. & 1972 & 359 & 8.87 & 2.63 & 9.63 & 1.44 & 94 \\
\hline 9211200. & 1973 & 365 & 8.09 & 2.49 & 8.70 & 1.42 & 93 \\
\hline 9211200 & 1974 & 365 & 9.27 & 2.60 & 7.34 & 1.42 & 95 \\
\hline 9211200. & 1975 & 365 & 8.28 & 2.47 & 8.65 & 1.76 & 91 \\
\hline 9211200 & 1976 & 366 & 9.25 & 2.59 & 9.59 & 1.73 & 92 \\
\hline 9217000 & 1951 & 147 & 10.75 & 2.71 & 7.10 & 1.98 & 61 \\
\hline 9217000 & 1952 & 296 & 11.37 & 2.80 & 8.00 & 2.51 & 89 \\
\hline 9217000 & 1953 & 349 & 10.89 & 2.76 & 9.53 & 2.30 & 91 \\
\hline 9217000. & 1954 & 355 & 9.55 & 2.74 & 8.92 & 2.15 & 90 \\
\hline 9217000. & 1953 & 356 & 10.23 & 2.77 & $8 . \overline{81}$ & 2.39 & 90 \\
\hline 9217000. & 1956 & 328 & 9.40 & 2.82 & 8.59 & 2.15 & 90 \\
\hline 9217000 & 1957 & 279 & 10.68 & 2.87 & 8.37 & 2.59 & 87 \\
\hline 9217000. & 1958 & 207 & 12.69 & 2.78 & 7.70 & 1.76 & 90 \\
\hline 9217000. & 1959 & 25.5 & 10.48 & 2.79 & 8.36 & 2.47 & 87 \\
\hline 9217000 & 1960 & 198 & 12.75 & 2.89 & 9.16 & 2.50 & 88 \\
\hline 9217000 & 1961 & 183 & 13.67 & 2.83 & 9.06 & 1.91 & 92 \\
\hline 9217000 & 1962 & 203 & 11.63 & 2.78 & 7.38 & 2.22 & 85 \\
\hline 9217000 & 1963 & 290 & 11.56 & 2.79 & 5.15 & 1.73 & 93 \\
\hline 9217000. & 1964 & 244 & 12.07 & 2.60 & 7.84 & 2.40 & 87 \\
\hline 9217000 & 1965 & 314 & 10.15 & 2.76 & 7.95 & 2.10 & 90 \\
\hline
\end{tabular}


Table 5.--Harmonic-function coefficients, Green River subbasin--Continued

\begin{tabular}{|c|c|c|c|c|c|c|c|}
\hline \multirow[b]{2}{*}{ Station no. ${ }^{2}$} & \multicolumn{7}{|c|}{ Harmonic coefficient ${ }^{1}$} \\
\hline & Year & NO & A & C & M & $\mathrm{SE}$ & PVAR \\
\hline 9217000 & 1966 & 294 & 11.75 & 2.76 & 8.90 & 1.90 & 93 \\
\hline 9217000 & 1967 & 344 & 10.16 & 2.75 & 8.63 & 2.02 & 92 \\
\hline 9217000 & 1968 & 313 & 10.07 & 2.81 & 8.32 & $2+18$ & 90 \\
\hline 9217000 & 1969 & 246 & 11.47 & 2.69 & 8.79 & 2.24 & 87 \\
\hline 9217000 & 1970 & 297 & 10.12 & 2.81 & 9.08 & 2.19 & 87 \\
\hline 9217000 & 1971 & 270 & 10.54 & 2.77 & 8.02 & 2.67 & 83 \\
\hline 9217000 & 1972 & 234 & 10.01 & 2.63 & 8.57 & 1.83 & 89 \\
\hline 9217000 & $197 \overline{3}$ & 250 & 10.18 & 2.79 & 7.14 & 2.27 & 83 \\
\hline 9217000 & 1974 & 274 & 10.59 & 2.77 & 7.43 & 2.65 & 84 \\
\hline 9217000 & 1975 & 283 & 10.06 & 2.60 & 8.08 & $2+34$ & 87 \\
\hline 9217000. & 1976 & 284 & 9.69 & 2.73 & 8.33 & 2.24 & 87 \\
\hline 9217000 & 1977 & 249 & 13.05 & 2.80 & 8.28 & 2.36 & 88 \\
\hline 9217000 & 1978 & 252 & 5.84 & 2.70 & 7.60 & 2.55 & 79 \\
\hline 9217000. & 1979 & 227 & 12.49 & 2.64 & 8.02 & 2.56 & 82 \\
\hline 9217000 & 1980 & 366 & 10.17 & 2.73 & 8.50 & 2.08 & 91 \\
\hline 9217000 & 1981 & 365 & 9.89 & 2.78 & 9.52 & 2.16 & 90 \\
\hline 9234500. & 1957 & 78 & $12 \cdot 25$ & 2.71 & 7.94 & 1.97 & 73 \\
\hline 9234500 & 1958 & 98 & 10.97 & 2.48 & 9.65 & 2.48 & 81 \\
\hline 9234500 & 1959 & 40 & 11.12 & 2.78 & B. 54 & 2.79 & 76 \\
\hline 9234500 & 1960 & 139 & 11.28 & 2.76 & 9.03 & 2.40 & 82 \\
\hline 9234500 & 1961 & 182 & 11.48 & 2.86 & 8.57 & 1.78 & 93 \\
\hline 9234500 & 1962 & 187 & 10.43 & 2.81 & 7.79 & 1.98 & 91 \\
\hline 9234500. & 1963 & 228 & 2.96 & 2.09 & 4.49 & 1.58 & 65 \\
\hline 9234500 & 1964 & 352 & 2.75 & 1.55 & 6.50 & 1.67 & 56 \\
\hline 9234500 & 1965 & 327 & 4.03 & 1.76 & 7.58 & .93 & 90 \\
\hline 9234500 & 1966 & 356 & 3.54 & .89 & 6.48 & 1.23 & 80 \\
\hline 9234500 & 1967 & 359 & 2.16 & 1.48 & 6.04 & .60 & 86 \\
\hline 9234500 & 1968 & 344 & 2.39 & 1.26 & 6.44 & .80 & 82 \\
\hline 9234500 . & 1969 & 333 & 3.14 & 1.26 & 6.56 & .91 & 85 \\
\hline 9234500 & 1970 & 274 & 2.85 & 1.26 & 6.43 & .82 & 86 \\
\hline 9234500 & 1971 & 251 & 2.30 & $1 \cdot 14$ & 6.0 .1 & .88 & 77 \\
\hline 9234500 & 1972 & 272 & 2.01 & 1.57 & 6.31 & .52 & 88 \\
\hline 9234500 & 1973 & 364 & 2.11 & .79 & 5.83 & .73 & 80 \\
\hline 9234500 & 1974 & 365 & 1.80 & 1.33 & 6.05 & .47 & 88 \\
\hline 9234500 & 1975 & 279 & 2.07 & 1.16 & 5.99 & .62 & 84 \\
\hline 9234500 & 1976 & 228 & 1.74 & 1.18 & 5.96 & .43 & 88 \\
\hline 9234500 & 1978 & 239 & 4.35 & 2.03 & 7.98 & 1.87 & 73 \\
\hline 9234500 & 1979 & 236 & 5.09 & 2.19 & 8.71 & .80 & 95 \\
\hline 9234500. & 1990 & 243 & 5.24 & 2.17 & 8.33 & .69 & 94 \\
\hline
\end{tabular}


Table 5.--Harmonic-function coefficients, Green River subbasin--Continued

\begin{tabular}{|c|c|c|c|c|c|c|c|}
\hline \multirow[b]{2}{*}{ Station no. ${ }^{2}$} & \multirow[b]{2}{*}{ Year } & \multicolumn{5}{|c|}{ Harmonic coefficient ${ }^{1}$} & \multirow[b]{2}{*}{ PVAR } \\
\hline & & NO & A & C & M & $\mathrm{SE}$ & \\
\hline 9234500 & 1991 & 239 & 4.49 & 2.19 & 8.33 & .78 & 92 \\
\hline 9261000 & 1950 & 360 & 10.36 & 2.68 & 10.74 & 1.77 & 94 \\
\hline 9201000 & 1951 & 356 & 9.71 & 2.72 & 11.11 & 1.36 & 76 \\
\hline 9261000 & 1952 & 343 & 9.47 & $2.6 \overline{1}$ & 10.34 & 1.43 & 95 \\
\hline 9261000 & 1953 & 314 & 10.25 & 2.74 & 9.55 & 2.05 & 92 \\
\hline 9261000 & 1754 & 291 & $11 \cdot 14$ & 2.86 & 10.98 & 2.19 & 92 \\
\hline 9261000 & 1955 & 259 & 10.70 & 2.74 & 9.49 & 1.66 & 95 \\
\hline 9261000 & 1956 & 258 & 9.63 & 2.83 & 9.36 & 1.95 & 91 \\
\hline 9261000. & 1957 & 263 & 10.61 & 2.91 & 9.55 & 1.92 & 94 \\
\hline 9261000 & 1958 & 177 & 12.08 & 2.80 & 10.81 & 2.20 & 92 \\
\hline 9261000 & 1959 & 167 & $11 \cdot 64$ & $2.8 B$ & 10.84 & 2.08 & 93 \\
\hline 9261000 & 1962 & 204 & 12.20 & 2.75 & 8.94 & 2.12 & $B 8$ \\
\hline 9261000 & 1963 & 301 & 11.18 & 2.74 & 11.43 & $2 \cdot 15$ & 91 \\
\hline 9261000 & 1964 & 223 & 9.64 & 2.70 & 9.55 & 2.01 & 86 \\
\hline 9261000 & 1965 & 215 & 9.13 & 2.69 & 9.36 & 1.89 & 89 \\
\hline 9261000 & 1966 & 211 & 8.66 & 2.72 & 10.17 & 1.74 & 89 \\
\hline 9261000 & 1967 & 213 & 8.43 & 2.78 & 9.96 & 1.79 & 87 \\
\hline 9261000 & 1968 & 272 & 8.03 & 2.85 & 10.45 & 1.84 & 89 \\
\hline 9261000 & 1969 & 285 & 9.00 & 2.84 & 10.57 & 1.27 & 96 \\
\hline 9261000 & 1970 & 264 & 8.81 & 2.79 & 10.53 & 1.73 & 92 \\
\hline 9261000. & 1971 & 235 & 8.80 & 2.88 & 9.07 & 1.69 & 92 \\
\hline 9261000 & 1972 & 233 & 8.94 & 2.89 & 10.07 & 1.84 & 91 \\
\hline 9261000 & 1973 & 218 & 8.49 & 2.80 & 9.51 & 1.78 & 90 \\
\hline 9261000 & 1974 & 201 & 8.92 & 2.83 & 9.55 & 1.98 & 87 \\
\hline 9261000 & 1975 & 201 & 8.25 & 2.71 & 9.06 & 1.75 & 90 \\
\hline 9261000. & 1976 & 135 & 8.69 & 2.90 & 9.56 & 1.53 & 92 \\
\hline 9261000. & 1977 & 260 & 8.54 & 2.80 & 10.42 & 2.16 & 89 \\
\hline 9261000. & 1978 & 290 & 9.72 & 2.79 & 11.86 & 2.09 & 91 \\
\hline 9261000. & 1979 & 253 & 11.89 & 2.80 & 11.29 & 2.13 & 92 \\
\hline 9261000. & 1980 & 309 & 9.32 & 2.66 & 10.40 & 2.40 & 87 \\
\hline 9261000 & 1981 & 310 & 9.94 & 2.84 & 12.54 & 1.76 & 93 \\
\hline 9307000 & 1951 & 156 & 11.88 & 2.75 & 11.62 & 1.99 & 94 \\
\hline 9307000 & 1952 & 114 & 16.26 & 2.60 & 7.85 & 2.08 & 93 \\
\hline 9307000. & 1954 & 302 & 11.84 & 2.83 & 12.36 & 2.12 & 94 \\
\hline 9307000 & 1955 & 63 & 12.84 & 2.67 & 10.72 & 2.04 & $8 B$ \\
\hline 9307000 & 1957 & 136 & 12.31 & 2.80 & 11.31 & 2.07 & 93 \\
\hline 9307000 & 1958 & 185 & 11.71 & 2.73 & 11.67 & 2.63 & 92 \\
\hline 9307000. & 1959 & 205 & $2 \cdot 64$ & 2.61 & 7.21 & 3.64 & 13 \\
\hline 9307000. & 1960 & 220 & 12.45 & 2.79 & 10.56 & 2.40 & 88 \\
\hline
\end{tabular}


Table 5.--Harmonic-function coefficients, Green River subbasin--Continued

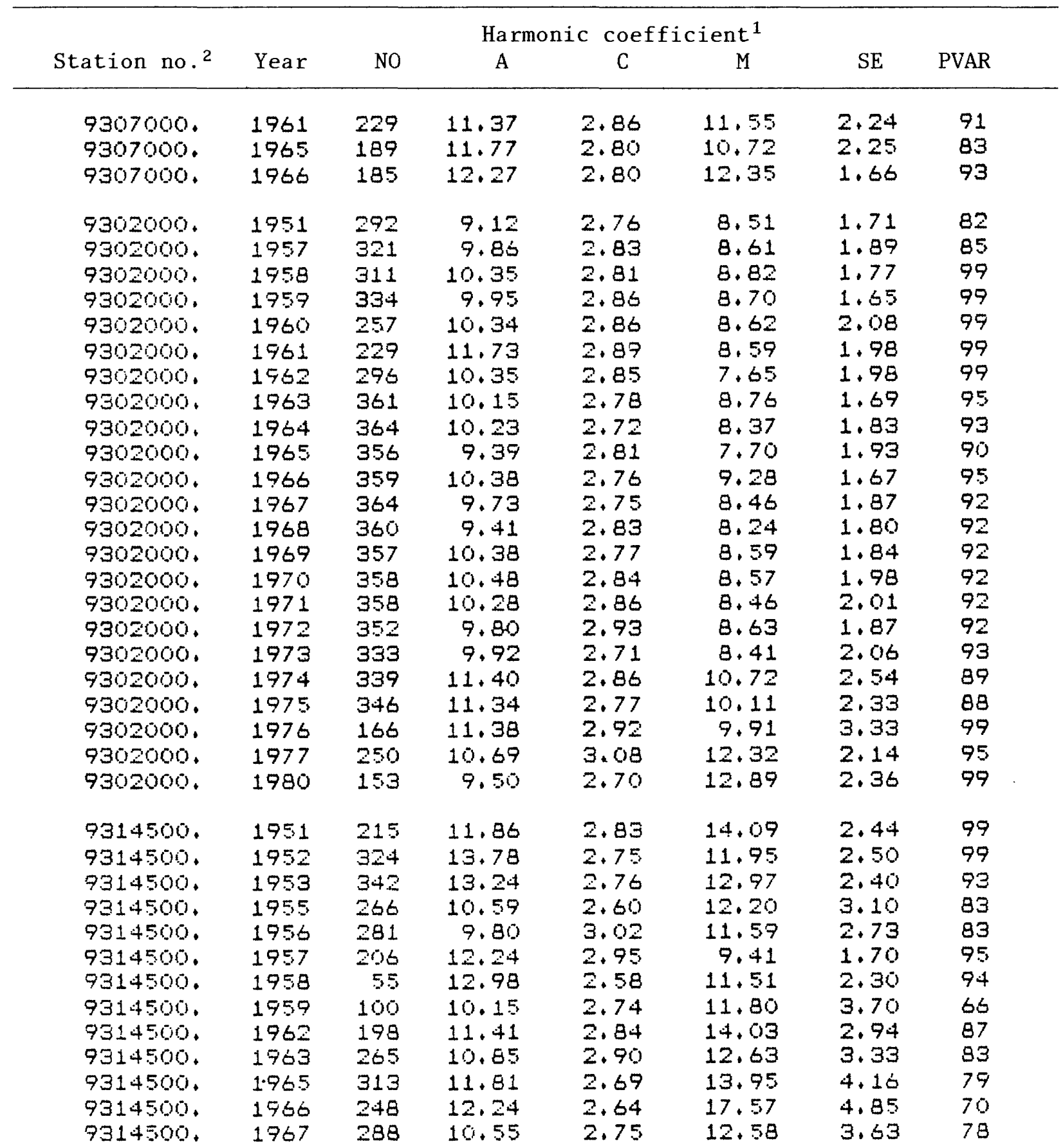


Table 5.--Harmonic-function coefficients, Green River subbasin--Continued

\begin{tabular}{|c|c|c|c|c|c|c|c|}
\hline \multirow[b]{2}{*}{ Station no. ${ }^{2}$} & \multicolumn{7}{|c|}{ Harmonic coefficient ${ }^{1}$} \\
\hline & Year & NO & $\mathrm{A}$ & C & M & SE & PVAR \\
\hline 9314500 & 1968 & 340 & 10.62 & 2.65 & 10.45 & 2.77 & 88 \\
\hline 9314500 & 1969 & 326 & 10.77 & 2.81 & 10.34 & $2+11$ & 92 \\
\hline 9314500 & 1970 & 301 & 8.83 & 2.82 & 8.37 & 2.91 & 78 \\
\hline 9314500 & 1971 & 289 & 10.87 & 2.88 & 9.01 & 3.14 & 82 \\
\hline 9314500 & 1972 & 308 & 10.79 & 2.92 & 9.98 & 2.68 & 87 \\
\hline 9314500 & $197 \overline{3}$ & 290 & 11.95 & 2.81 & 10.54 & 2.80 & 87 \\
\hline 9314500 & 1974 & 283 & 9.22 & 2.95 & 9.46 & 3.23 & 74 \\
\hline 9314500 & 1975 & 277 & 10.72 & 2.77 & 9.96 & 2.93 & 84 \\
\hline 9314500 & 1976 & 352 & 10.34 & 3.06 & 11.08 & 2.60 & 88 \\
\hline 9314500 & 1978 & 308 & 12.84 & 2.87 & 13.86 & 2.70 & 91 \\
\hline 9315000. & 1949 & 137 & 11.26 & 2.55 & $12+83$ & 1.69 & 79 \\
\hline 9315000 & 1950 & 321 & 11.19 & 2.74 & 11.73 & 1.86 & 94 \\
\hline 9315000 & 1951 & 304 & 11.50 & 2.80 & 12.48 & 2.06 & 94 \\
\hline 9315000 & 1952 & 288 & 12.48 & 2.73 & 11.49 & 1.93 & 95 \\
\hline 9315000 & 1953 & 299 & 11.65 & 2.73 & $12 \cdot 28$ & 2.13 & 93 \\
\hline 9315000 & 1954 & 342 & 11.79 & 2.84 & 13.13 & 2.29 & 92 \\
\hline 9315000 & 1755 & 277 & 13.21 & 2.66 & 11.53 & 1.62 & 96 \\
\hline 9315000. & 1956 & 331 & 11.75 & 2.79 & 12.24 & 1.80 & 95 \\
\hline 9315000. & 1957 & 291 & 11.30 & 2.79 & 11.49 & 2.44 & 90 \\
\hline 9315000. & 1958 & 298 & 12.33 & 2.80 & 13.75 & 2.08 & 94 \\
\hline 9315000 & 1959 & 218 & 10.61 & 2.78 & 14.29 & 1.90 & 92 \\
\hline 9315000 & 1960 & 194 & 12.94 & 2.81 & 13.23 & 1.99 & 94 \\
\hline 9315000 & 1961 & 84 & 12.04 & 2.88 & 13.85 & 2.09 & 93 \\
\hline 9315000 & 1962 & 196 & 12.69 & 2.80 & 12.41 & 2.30 & 90 \\
\hline 9315000 & 1963 & 138 & 12.17 & 2.82 & 14.07 & 2.38 & 88 \\
\hline 9315000 & 1964 & 214 & 11.55 & 2.74 & 12.16 & 2.66 & 85 \\
\hline 9315000 & 1965 & 165 & 12.42 & 2.77 & 10.50 & 2.09 & 91 \\
\hline 9315000. & 1966 & 176 & $12 \cdot 13$ & 2.85 & 13.16 & 1.76 & 75 \\
\hline 9315000 & 1967 & 274 & 10.57 & 2.71 & 10.96 & 2.63 & 87 \\
\hline 9315000 & 1968 & 263 & 10.68 & 2.82 & 10.39 & 1.89 & 91 \\
\hline 9315000 & 1969 & 267 & 10.27 & 2.71 & 10.19 & 1.41 & 95 \\
\hline 9315000 & 1970 & 277 & 10.69 & 2.87 & 10.53 & 1.77 & 94 \\
\hline 9315000 & 1971 & 286 & 10.81 & 2.87 & 10.56 & 1.68 & 95 \\
\hline 9315000 & 1972 & 277 & 10.80 & 2.72 & $12 \cdot 23$ & 1.95 & 93 \\
\hline 9315000 & 1973 & 257 & 11.68 & 2.76 & 11.12 & 1.77 & 95 \\
\hline 9315000 & 1974 & 227 & 11.95 & 2.88 & 12.60 & 3.23 & 82 \\
\hline 9315000 & 1975 & 234 & 10.77 & 2.73 & 11.65 & 2.06 & 92 \\
\hline 9315000 & 1976 & 265 & 11.68 & 2.83 & 12.49 & 2.19 & 92 \\
\hline 9315000 & 1977 & 305 & 13.13 & 2.83 & 13.36 & 1.96 & 95 \\
\hline
\end{tabular}


Table 5.--Harmonic-function coefficients, Green River subbasin--Continued

\begin{tabular}{|c|c|c|c|c|c|c|c|}
\hline \multirow[b]{2}{*}{ Station no. ${ }^{2}$} & \multirow[b]{2}{*}{ Year } & \multirow[b]{2}{*}{ NO } & \multicolumn{3}{|c|}{ Harmonic Coefficient ${ }^{1}$} & \multirow[b]{2}{*}{$\mathrm{SE}$} & \multirow[b]{2}{*}{ PVAR } \\
\hline & & & $\mathrm{A}$ & C & M & & \\
\hline 9315000 & 1978 & 285 & 11.67 & 2.82 & 13.34 & 2.15 & 93 \\
\hline 9315000 & 1979 & 234 & 12.92 & 2.77 & 12.51 & 2.02 & 93 \\
\hline 9315000 & 1980 & 320 & 10.00 & 2.76 & 12.84 & 2.42 & 87 \\
\hline 9315000 & 1981 & 309 & 11.79 & 2.88 & 13.05 & 2.02 & 94 \\
\hline 9328500 & 1951 & 281 & 12.09 & 2.88 & 13.96 & 3.16 & 87 \\
\hline 9328500 & 1952 & 250 & 12.47 & 2.74 & 12.64 & 3.23 & 86 \\
\hline 9328500 & 1953 & 250 & 10.81 & 2.73 & 13.61 & 2.70 & 86 \\
\hline 9328500 & 1954 & 283 & 13.33 & 2.94 & 13.93 & 2.63 & 92 \\
\hline 9328500 & 1955 & 222 & 12.89 & 2.76 & 12.99 & 2.81 & 87 \\
\hline 9328500 & 1956 & 218 & 12.46 & 3.01 & 12.95 & 2.52 & 93 \\
\hline 9328500 & 1957 & 276 & 11.12 & 2.92 & $12+11$ & 3.57 & 82 \\
\hline $\begin{array}{l}9328500 \\
9328500\end{array}$ & $\begin{array}{l}1958 \\
1959\end{array}$ & $\begin{array}{l}214 \\
262\end{array}$ & $\begin{array}{r}12.90 \\
6.52\end{array}$ & $\begin{array}{l}2.77 \\
2.57\end{array}$ & $\begin{array}{l}12.29 \\
13.01\end{array}$ & $\begin{array}{l}2.87 \\
5.71\end{array}$ & $\begin{array}{l}88 \\
35\end{array}$ \\
\hline 9328500 & 1960 & 155 & $12 \cdot 31$ & 2.80 & 11.06 & 3.10 & 80 \\
\hline 7328500 & 1961 & 177 & 13.77 & 2.81 & 9.85 & 2.92 & 86 \\
\hline 9328500 & 1962 & 207 & 11.38 & 2.85 & 10.74 & 2.91 & 84 \\
\hline 9328500 & 1963 & 204 & 11.98 & 2.93 & 13.83 & 2.98 & 86 \\
\hline 9328500 & 1964 & 200 & 11.94 & 2.72 & 11.33 & 2.85 & 87 \\
\hline 9328500 & 1965 & 198 & 10.60 & 2.68 & 10.99 & 3.15 & 82 \\
\hline 7328500 & 1766 & 166 & $12 \cdot 10$ & 2.83 & 12.30 & 2.49 & 89 \\
\hline 9328500 & 1967 & 207 & 11.17 & 2.76 & 11.01 & 2.75 & 82 \\
\hline 9328500. & 1958 & 234 & 10.02 & 2.79 & 10.81 & 2.67 & 82 \\
\hline 9328500 & 1967 & 232 & 11.10 & 2.92 & 11.63 & 2.95 & 82 \\
\hline 9328500 & 1770 & 156 & 9.94 & 2.99 & 12.01 & 2.98 & 78 \\
\hline 9328500. & 1971 & 98 & 10,76 & 2.87 & 11.27 & 3.15 & 83 \\
\hline 9328500 & 1972 & 82 & 12.19 & 2.95 & 12.39 & 3.08 & 83 \\
\hline 9328500. & 1773 & 144 & 11,86 & 2.74 & $11 \cdot 22$ & 3.07 & 73 \\
\hline 9328500 & 1974 & 212 & 12.85 & 2.76 & 13.39 & 3.41 & 92 \\
\hline 9328500 & 1975 & 172 & 11.73 & 2.78 & 12.32 & 2.83 & 86 \\
\hline 9328500 & 1976 & 113 & 12.81 & 2.95 & $13 \cdot 40$ & 3.09 & 88 \\
\hline 9329500 & 1977 & 57 & 14.02 & 3.03 & 14.41 & 3.75 & 84 \\
\hline
\end{tabular}

${ }^{1}$ See equation (1), p. 2 of text.

${ }^{2}$ See supplementary information for station description. 
Corresponding seasonal patterns at these sites during the 1976 water year are depicted in figure 5. As one proceeds in a downstream direction, the harmonic mean would be expected to increase. Observed anomalies from this expected pattern are caused by Flaming Gorge Reservoir (between station 09217000 Green River near Green River, Wyo., and station 09234500 Green River near Greendale, Utah) and Fontenelle Reservoir (between station 09209400 Green River near LaBarge, Wyo., and station 09211200 Green River near Fontenelle Reservoir, Wyo.). Nonetheless, between the uppermost main-stem site (station 09188500 Green River at Warren Bridge, near Daniel, Wyo.) and the lowermost site (station 09315000 Green River at Green River, Utah), harmonic mean temperatures increase from a range of 5.1 to $5.6^{\circ} \mathrm{C}$ upstream (partial year results for the 1962 water year in table 5 should be ignored), to 10.2 to $13.8^{\circ} \mathrm{C}$ downstream; corresponding amplitudes increase from 5.7 to $5.8^{\circ} \mathrm{C}$ upstream to 10.3 to $13.2^{\circ} \mathrm{C}$ downstream. Water-temperature characteristics of station 09314500 Price River at Woodside, Utah; station 09302000 Duchesne River near Randlett, Utah; and station 09328500 San Rafael River near Green River, Utah; are similar to those for station 09315000 Green River at Green River, Utah. Harmonic coefficients for three additional smaller tributaries in the Wyoming part of the Green River subbasin have been reported by Lowham (1978, table 4).

\section{Yampa River Subbasin}

Fifty-nine years of daily records were available for three sites in the Yampa River subbasin. The two long-term records are for sites located in downstream reaches of the Yampa River and the Little Snake River. For purposes of the time-trend analysis for the Little Snake River, records for station 09259950 Little Snake River above Lily, Colo., and station 09260000 Little Snake River near Lily, Colo., were combined (Wentz and Steele, 1980). No significant trends in these two records were detected, as expected, because of only minor reservoir regulation of streamflows to date of the Yampa River system (Steele and others, 1979; Wentz and Steele, 1980). Recently, monitoring instrumentation has been operating at station 09260025 Yampa River below the Little Snake River, Colo. In evaluating resultant coefficients for the 1979 water year, seasonal water-temperature patterns are intermediate to those for the two upstream measurement sites (table 6 and fig. 6).

For comparative purposes, harmonic coefficients for intermittent watertemperature measurements for the 1975 water year are given (table 6) as reported by Briggs and Ficke (1978); however, the resultant phase-angle coefficient for the Little Snake River near Lily appears somewhat anomalous (table 6). Stream-reach profiles for both the Yampa River and the Little Snake River, using intermittent water-temperature measurements for several main-stem sites not included in this study, showed characteristic increases in a downstream direction of harmonic-mean temperatures, and, to a lesser extent, harmonic amplitudes (Steele and others, 1979, fig. 2).

\section{Colorado River at Lees Ferry, Ariz.}

Thirty-two years of daily records were available at station 09380000 Colorado River at Lees Ferry, Ariz. A significant decrease in all three 


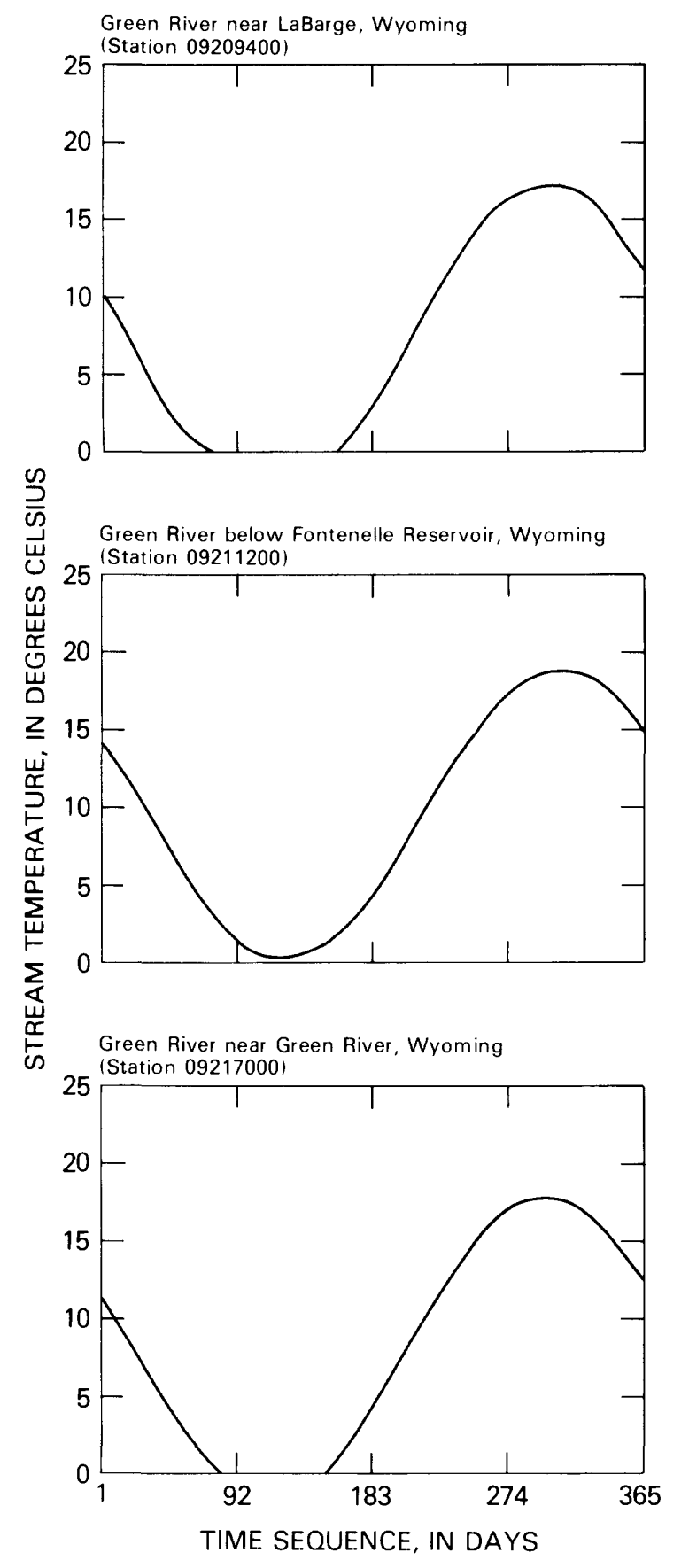

Green River near Greendale, Utah (Station 09234500)

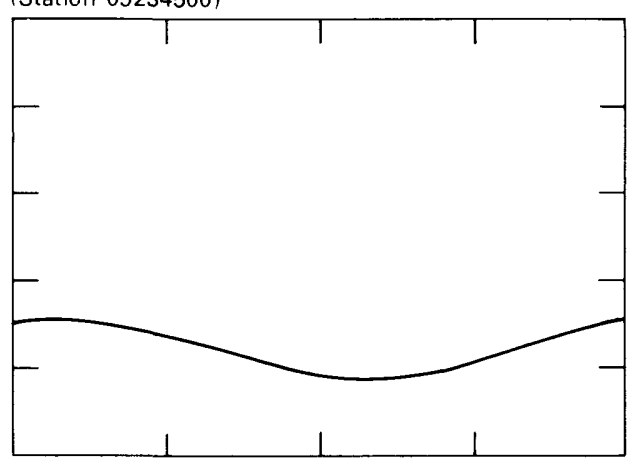

Green River at Jensen, Utah (Station 09261000)

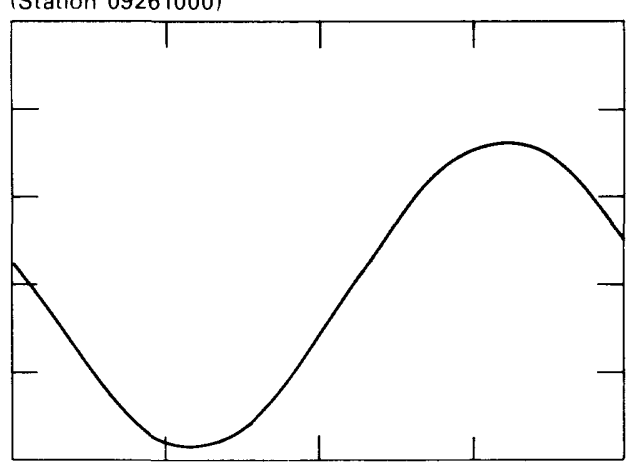

Green River at Green River, Utah (Station 09315000)

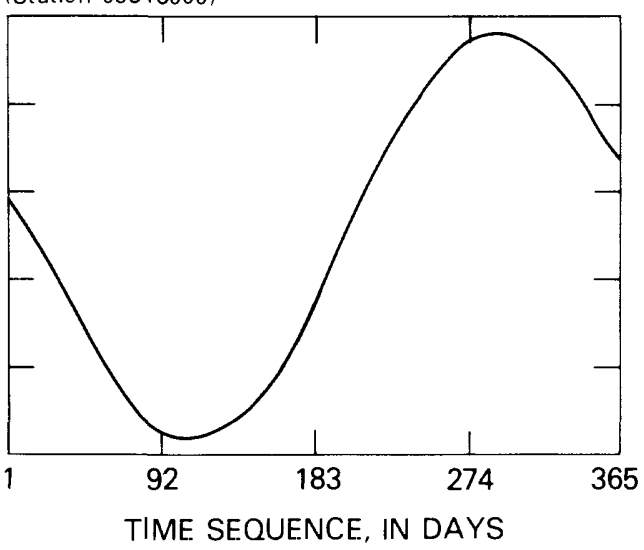

Figure 5.--Stream-reach seasonal water-temperature patterns, Green River, 1976 water year. 
Table 6.--Harmonic-function coefficients, Yampa River subbasin

[NO = number of daily values; $\mathrm{SE}=$ standard error of estimate; $P V A R=$ percent variance explained by the harmonic function]

\begin{tabular}{|c|c|c|c|c|c|c|c|}
\hline \multirow[b]{2}{*}{ Station no. ${ }^{2}$} & \multicolumn{7}{|c|}{ Harmonic coefficients ${ }^{1}$} \\
\hline & Year & NO & A & $\mathrm{C}$ & M & $\mathrm{SE}$ & PVAR \\
\hline 9251000 & 1951 & 293 & 10.52 & 2.62 & 10.50 & 2.20 & 92 \\
\hline 9251000 & 1952 & 340 & 11.57 & 2.65 & 9.74 & 1.98 & 94 \\
\hline 9251000 & 1953 & 354 & 12.08 & 2.55 & 10.76 & 2.61 & 51 \\
\hline 9251000 & 1954 & 313 & $12 \cdot 85$ & 2.74 & 10.64 & 2.00 & 94 \\
\hline 9251000 & 1955 & 361 & 12.30 & 2.57 & 9.91 & 2.23 & 53 \\
\hline$\$ 251000$ & 1956 & 354 & 11.75 & 2.67 & 9.98 & 2.06 & 94 \\
\hline 9251000 & 1957 & 280 & 10.60 & 2.59 & 9.02 & 3.01 & 82 \\
\hline 9251000 & 1958 & 287 & 11.87 & 2.69 & 10.20 & 2.50 & 89 \\
\hline 9251000 & 1959 & 282 & 9.92 & 2.71 & 10.49 & 2.54 & 86 \\
\hline 9251000 & 1960 & 281 & 11.19 & 2.73 & 9.66 & 2.87 & 86 \\
\hline 9251000 & 1961 & 238 & 11.26 & 2.70 & 10.45 & 2.72 & 87 \\
\hline 9251000 & 1962 & 293 & 11.10 & 2.71 & 9.43 & 2.34 & 90 \\
\hline 9251000 & 1963 & 361 & 11.00 & 2.73 & 10.58 & 2.38 & 91 \\
\hline 9251000 & 1964 & 357 & 9.76 & 2.44 & 8.86 & 2.46 & 88 \\
\hline 9251000 & 1965 & 281 & 9.35 & 2.45 & 8.59 & 2.55 & 85 \\
\hline 9251000 & 1966 & 301 & 8.26 & 2.50 & 9.10 & 3.54 & 67 \\
\hline 9251000 & 1967 & 338 & 9.99 & 2.56 & 10.03 & 2.59 & 84 \\
\hline 9251000 & 1968 & 363 & 8.87 & 2.55 & 8.35 & 2.83 & 83 \\
\hline 9251000 & 1969 & 349 & 12.00 & 2.60 & 10.35 & 2.89 & 87 \\
\hline 9251000 & 1970 & 364 & 10.50 & 2.54 & 10.11 & 2.77 & 89 \\
\hline 9251000 & 1971 & 363 & 11.60 & 2.43 & 10.57 & 2.57 & 91 \\
\hline 9251000 & 1972 & 358 & 10.75 & 2.78 & 5.99 & 3.35 & 83 \\
\hline 9251000 & 1973 & 304 & 5.75 & 2.74 & 9.40 & 2.39 & 88 \\
\hline 9251000 & 1975 & 54 & 11.50 & 2.84 & 8.73 & 1.87 & 90 \\
\hline 9251000 & 1976 & 114 & 13.40 & 2.83 & 8.5 .5 & $1+5 \theta$ & 95 \\
\hline 9251000 & 1977 & 177 & 15.19 & 2.84 & 9.55 & 2.15 & 91 \\
\hline 5251000 & 1978 & 163 & 5.86 & 2.29 & 11.22 & 2.56 & 74 \\
\hline 5251000 & 1979 & 315 & 10.50 & 2.66 & 8.45 & 2.19 & 96 \\
\hline 5251000 & 1980 & 235 & 12.27 & 2.87 & 10.50 & 1.30 & 97 \\
\hline 9251000 & 1981 & 363 & 10.74 & 2.86 & 9.65 & 1.98 & 92 \\
\hline 9259950 & 1951 & 180 & 9.34 & 2.84 & $9+28$ & 1.96 & 91 \\
\hline 9259950 & 1752 & $12 \theta$ & 9.50 & 2.89 & 6.52 & 1.91 & 82 \\
\hline 9255950 & 1953 & 241 & 10.13 & 2.90 & 6.35 & 2.28 & 83 \\
\hline 9259950 & 1954 & 238 & 9.32 & 2.98 & 7.12 & 2.12 & 85 \\
\hline 9259950 & 1955 & 234 & 12.75 & 2.76 & 6.55 & 3.16 & 79 \\
\hline 9259950 & 1956 & 235 & 14.97 & 2.83 & $7 \cdot 30$ & 3.14 & 85 \\
\hline 9259950 & 1957 & 255 & $11+10$ & 2.72 & 10.37 & 3.41 & 81 \\
\hline 5239950 & 1558 & 265 & 11.54 & 2.78 & 10.32 & 3.40 & 79 \\
\hline 5259950 & 1959 & 279 & 12.16 & 2.84 & 10.59 & 3.16 & 86 \\
\hline
\end{tabular}


Table 6.--Harmonic-function coefficients, Yampa River subbasin--Continued

\begin{tabular}{|c|c|c|c|c|c|c|c|}
\hline \multirow[b]{2}{*}{ Station no. ${ }^{2}$} & \multirow{2}{*}{\multicolumn{3}{|c|}{$\begin{array}{c}\text { Harmonic } \\
\text { A }\end{array}$}} & \multicolumn{2}{|c|}{ coefficients $^{1}$} & \multirow[b]{2}{*}{ SE } & \multirow[b]{2}{*}{ PVAR } \\
\hline & & & & $\mathrm{C}$ & M & & \\
\hline $72599=0$ & 1700 & $20 \%$ & $1 \overline{3} .46$ & 2.30 & $16+45$ & 3.12 & $\Xi \dot{b}$ \\
\hline $9257=56$ & -761 & 239 & $\pm 1 .=8$ & $\because .57$ & 10.07 & 4.19 & 75 \\
\hline 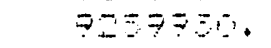 & 1952 & 175 & $13=1$ & ¿. 54 & $\Xi .75$ & $\therefore 91$ & 70 \\
\hline $5=5950$ & 1703 & 173 & $1 \pm+\infty$ & \pm .75 & $\bar{T}, \bar{E}$ & 3.17 & a1 \\
\hline 7230750 & $17=4$ & 132 & \pm+50 & $=.57$ & 9.11 & 3.55 & $E z$ \\
\hline $92=7556$ & 1905 & 175 & $\pm=5$ & $z+E$ & 7. & $\equiv, 5$ & 71 \\
\hline $72 \equiv=750$ & \pm 706 & $1=$ & 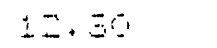 & $2 \cdot 3=$ & $\therefore \therefore 三$ & $=.71$ & $\Xi$ \\
\hline 7550950 & $19 \leq 7$ & $=$ & $\therefore \therefore \leq 1$ & 2.77 & $1 i+40$ & $\Xi . \Xi 7$ & 75 \\
\hline $92=0=0$ & 1703 & 176 & $1=82$ & \pm .75 & $7.2 \equiv$ & $\Xi .2 \equiv$ & 77 \\
\hline $725995 \%$ & $-\overline{7}$ & $14=$ & 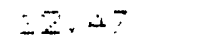 & 2.82 & $7.5 e$ & $3+26$ & 20 \\
\hline F2ப60\%, & $\therefore a z$ & $7 \pm$ & 10.71 & $1.7 ?$ & 11.05 & 5.10 & 51 \\
\hline 9250000 & \pm 970 & 260 & $\therefore=0$ & 2.51 & $9.2 E$ & 1.56 & 87 \\
\hline 7250900 & $177 i$ & 150 & $8+4$ & 2.90 & $9: 75$ & 2.34 & 80 \\
\hline 526001 & \pm 975 & Ẽo & $9+3$ & \pm .55 & 7.13 & $2.3 \Xi$ & 87 \\
\hline 7250000 & 17,9 & 182 & $10 \cdot 2$ & 2.52 & $7 . \leq 1$ & \pm .81 & $\Xi \Xi$ \\
\hline 7200000. & 1500 & 114 & $7.7=$ & 2.7 & 7.61 & $1.5 i$ & 99 \\
\hline $926000 \%$ & $1 戸 \boxminus \pm$ & $3+6$ & $10 \cdot \dot{0}$ & $=.73$ & $\bar{y} . \bar{z}$ & 1.25 & $5 \Xi$ \\
\hline $92090=$ & 1778 & $=72$ & 7.57 & 2.83 & $7+4 z$ & 275 & $E 0$ \\
\hline 7260025 & 1977 & 304 & 10.76 & 2,71 & 5.72 & 1.77 & $\overline{5}$ \\
\hline$=200=$ & 1550 & Eบ & $10+70$ & $2.8 \Xi$ & 8.75 & 1.77 & $5 \%$ \\
\hline
\end{tabular}

${ }^{1}$ See equation (1), p. 2 of text.

${ }^{2}$ See supplementary information for station description

harmonic coefficients and the amplitude plus mean coefficient began around the 1963 water year (table 7). This coincided with the completion and closure of Glen Canyon Dam and Reservoir. For several years (1974, 1977, and 1979 through 1981 water years), the harmonic-function results are not meaningful. Harmonic analyses of data for all these years have less than 50 percent variance explained, which would further confirm that the normal seasonal variability of stream temperatures at this site has been altered in recent years through upstream reservoir development. This change in the prereservoir versus post-reservoir downstream water temperature characteristics, depicted in figure 7 , is discussed in the next section. 


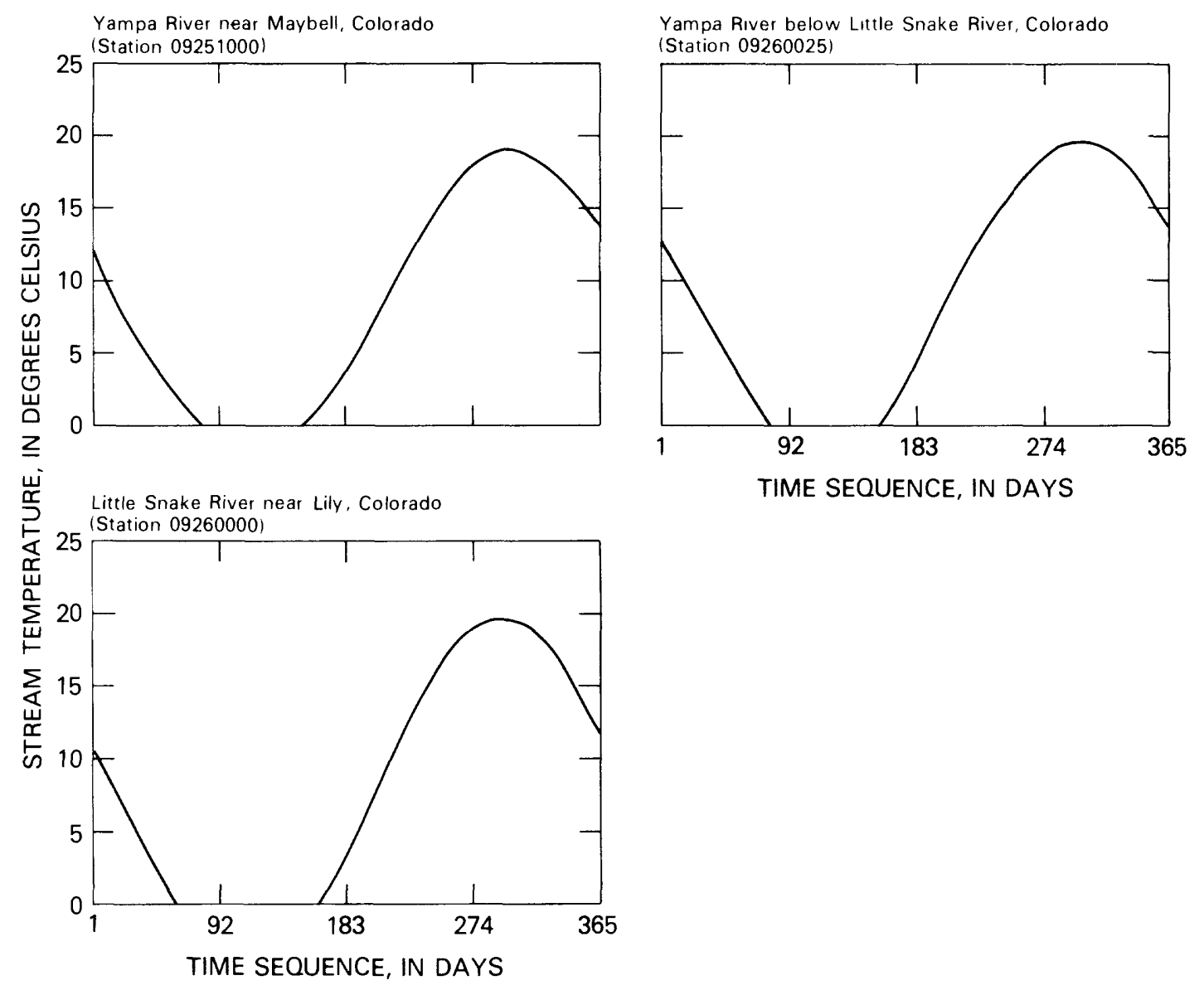

Figure 6.--Seasonal water-temperature patterns, Yampa River subbasin, 1979 water year. 
Table 7.--Harmonic-function coefficients, Colorado River at Lees Ferry, Ariz.

[NO = number of daily valued; $\mathrm{SE}=$ standard error of estimate; PVAR = percent variance explained by the harmonic function]

\begin{tabular}{|c|c|c|c|c|c|c|c|}
\hline \multirow[b]{2}{*}{ Station no. ${ }^{2}$} & \multirow[b]{2}{*}{ Year } & \multicolumn{4}{|c|}{ Harmonic coefficient ${ }^{1}$} & \multirow[b]{2}{*}{$\mathrm{SE}$} & \multirow[b]{2}{*}{ PVAR } \\
\hline & & NO & A & $\mathrm{C}$ & M & & \\
\hline 9380000 & 1950 & 358 & 10.71 & 2.81 & 13.79 & 2.07 & 93 \\
\hline 9380000 & 1951 & 354 & 10.87 & 2.75 & 14.19 & 1.91 & 94 \\
\hline 9380000 & 1952 & 359 & 11.43 & 2.74 & 12.91 & 1.96 & 94 \\
\hline 9380000 & 1953 & 276 & $11 \cdot 21$ & 2.69 & 14.90 & 1.99 & 86 \\
\hline 9380000 & 1954 & 319 & 11.89 & 2.88 & 14.84 & 1.99 & 93 \\
\hline 9380000 & 1955 & 353 & 11.94 & 2.69 & $13 \cdot 34$ & 1.76 & 95 \\
\hline 9380000 . & 1956 & 320 & 10.48 & 2.77 & 14.13 & 1.67 & 97 \\
\hline 9380000 & 1957 & 323 & 9.69 & 2.87 & 13,38 & 2.57 & 86 \\
\hline 7380000 & 195,8 & 314 & 10.82 & 2.77 & 14.15 & 3.17 & 83 \\
\hline 9380000 & 1959 & 308 & 11.67 & 2.84 & 14.54 & 1.90 & 93 \\
\hline 9380000. & 1960 & 278 & 11.80 & 2.82 & 14.29 & 2.49 & 90 \\
\hline 9390000 & 1961 & 274 & 11.80 & 2.86 & 14.55 & 1.60 & 99 \\
\hline 9380000 & 1962 & 263 & 11.58 & 2.77 & 13.40 & 2.09 & 97 \\
\hline 9380000 & 1963 & 245 & 7.92 & 2.57 & 11.65 & 2.11 & 87 \\
\hline 9380000 & 1964 & 210 & 5.61 & 1.83 & 12.50 & 2.10 & 64 \\
\hline 9380000. & 1965 & 190 & 5.83 & 1.93 & 14.68 & 1.40 & 98 \\
\hline 9380000. & 1966 & 217 & 4.56 & 1.35 & 14.49 & .94 & 92 \\
\hline 9380000 & 1967 & 331 & 6.89 & 2.19 & 12.70 & 1.04 & 94 \\
\hline 9380000. & 1948 & 244 & 7.05 & 2.32 & 13.87 & 1.07 & 99 \\
\hline 9380000 & 1969 & 257 & 5.14 & 1.95 & 12.55 & 1.22 & 89 \\
\hline 9380000 & 1970 & 299 & 4.57 & 2.22 & $11 . \equiv 0$ & 1.36 & 80 \\
\hline 9380000 & 1971 & 293 & 3.10 & 1.89 & 10.90 & 1.29 & 63 \\
\hline 9380000 & 1972 & 260 & 2.02 & 2.82 & 10.22 & .56 & 64 \\
\hline 9380000 & 1973 & 301 & 1.66 & 1.46 & 8.52 & .57 & 77 \\
\hline 9380000 & 1974 & 303 & .27 & .83 & 8.45 & .18 & 7 \\
\hline 9380000 & 1975 & 278 & 1.06 & 2.61 & 8.66 & .46 & 64 \\
\hline 9390000 & 1976 & 355 & .90 & 2.17 & 9.21 & .22 & 58 \\
\hline 9380000 & 1977 & 306 & .42 & 4.10 & 9.72 & .30 & 8 \\
\hline 9380000 & 1978 & $31 e$ & 1.61 & 2.59 & 9.80 & .53 & $7 \overline{9}$ \\
\hline 9380000 & 1979 & 346 & .75 & .55 & 9.47 & .46 & 25 \\
\hline 9380000 & 1980 & 308 & .77 & 2.05 & 8.41 & .20 & 29 \\
\hline 9380000 & 1981 & 268 & .53 & 2.76 & 10.07 & .28 & 37 \\
\hline
\end{tabular}

${ }^{1}$ See equation (1), p. 2 of text.

${ }^{2}$ See supplementary information for station description. 

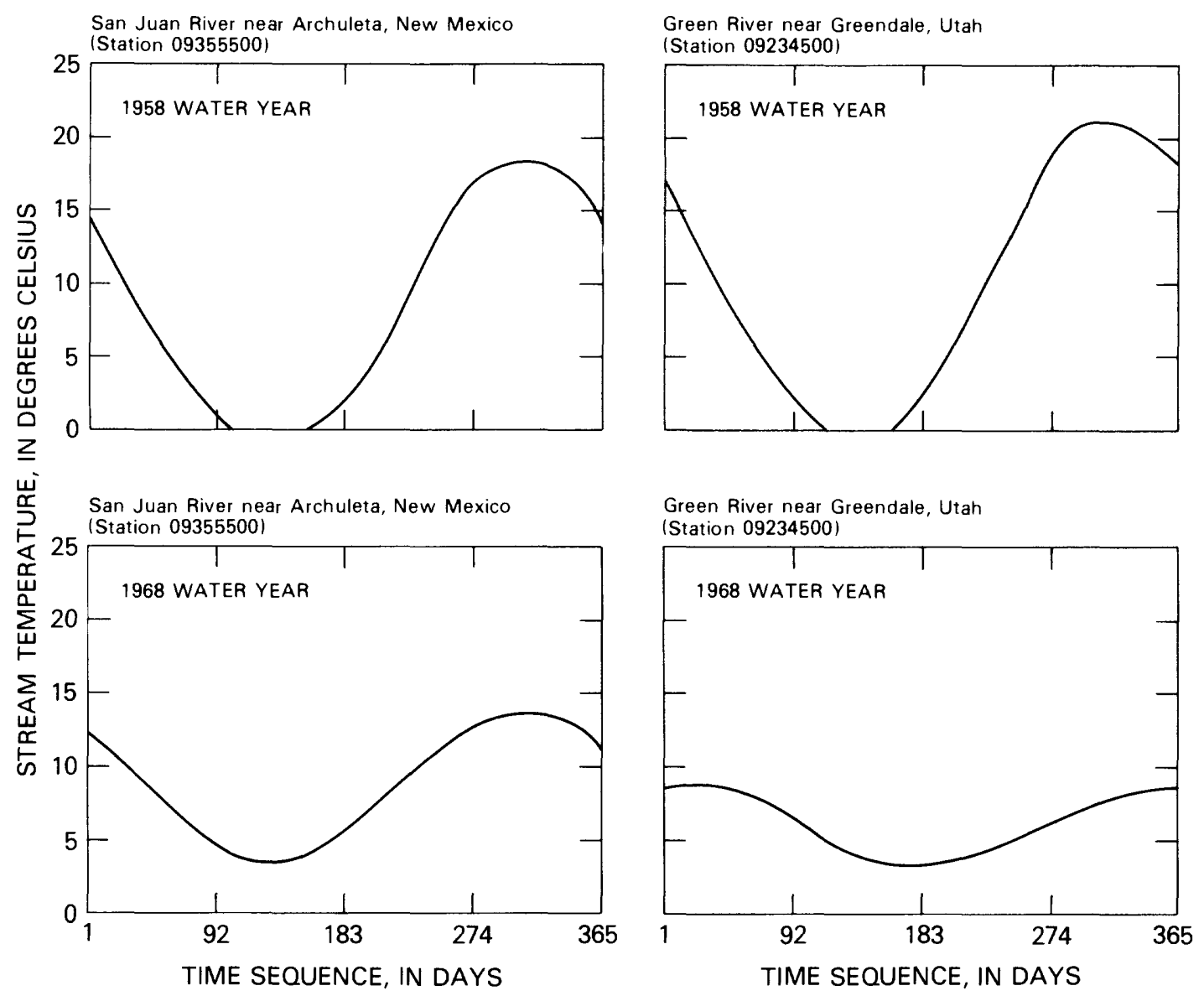

Figure 7.--Changes in seasonal water-temperature patterns, 1958 water year versus 1968 water year. 


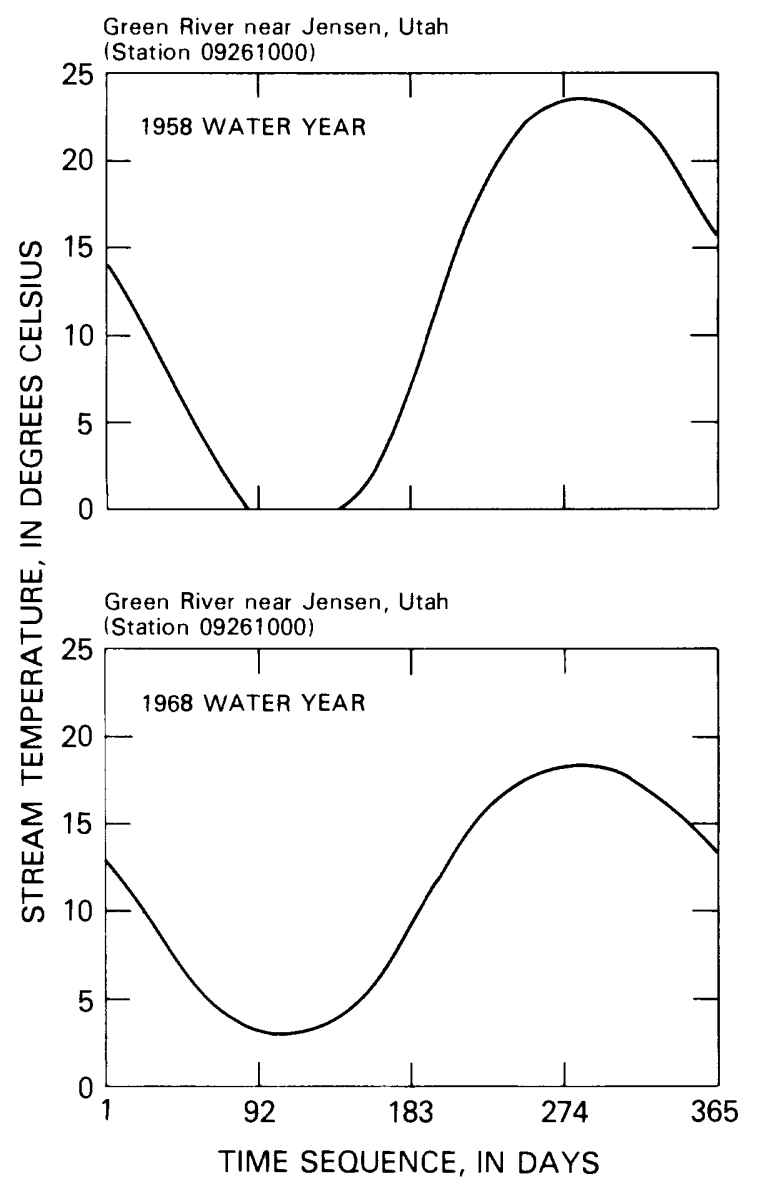

Colorado River at Lee's Ferry, Arizona (Station 09380000)

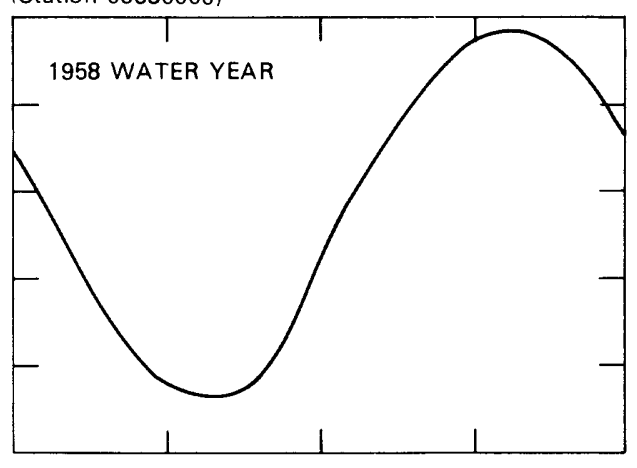

Colorado River at Lee's Ferry, Arizona (Station 09380000)

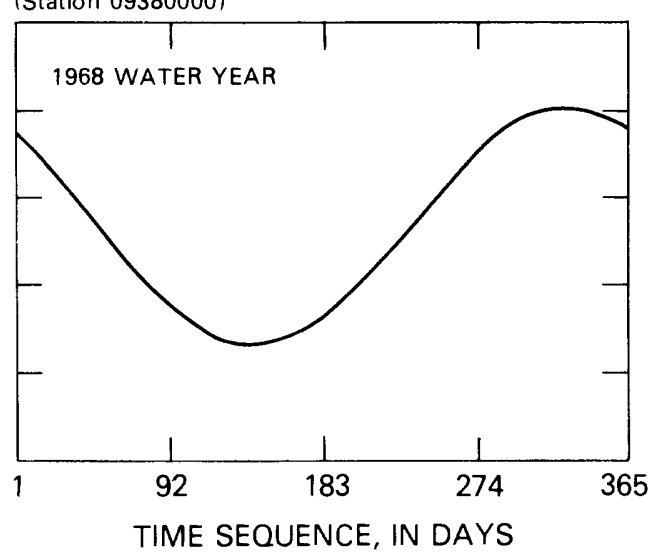

Figure 7.--Changes in seasonal water-temperature patterns, 1958 water year versus 1968 water year--Continued. 


\section{Impacts of Reservoirs on Water Temperature}

The Kendall's tau test (Conover, 1971) was applied to each annual time series of harmonic coefficients to statistically determine if there were trends in the series. The level of significance was assumed at the 99-percent confidence level, using the same procedures as outlined by Steele and others (1974). Finally, causal effects for observed trends were sought.

Significant trends were found in one or more coefficients for only 6 of the 26 station records on major streams in the Upper Colorado River Basin with 8 or more years of data (table 8). One site was station 09306500 White River near Watson, Utah; the second site was station 09095500 Colorado River near Cameo, Colo. No explanation is provided at this time for the two significant trends in stream-temperature harmonic coefficients. The third and fourth sites were station 09355500 San Juan River near Archuleta, N. Mex., and station 09365000 San Juan River at Farmington, N. Mex. The fifth site was station 09234500 Green River near Greendale, Utah, and the sixth site was station 09380000 Colorado River at Lees Ferry, Ariz. Selected examples of shifts in seasonal patterns are depicted in figure 7.

In the case of the station 09355500 San Juan River near Archuleta record, only the amplitude coefficient showed a significant trend (see table 4); the shift was considered caused by the operating of Navajo Reservoir beginning about 1963. The record at the next measurement site downstream, station 09357000 San Juan River at Bloomfield, did not extend beyond the 1963 water year; the record for the San Juan River at Farmington began in 1964 . Hence, no corresponding shift in time would have been expected. Nonetheless, in the latter case, a significant trend in the harmonic mean was observed, even though the reservoir is located upstream from the Bloomfield site (table 8 ).

For the Green River downstream from Flaming Gorge Reservoir, water is released from depth, so that the time series of amplitude coefficients have changed significantly to smaller values at station 09234500 Green River near Greendale, Utah, measurement site. Coefficient changes are apparent in the annual time series at this site beginning in 1963 (table 5 and fig. 7).

Downstream at station 09261000 Green River near Jensen measurement site, some decrease in the harmonic-amplitude coefficient remains, but it was not significant at the 99-percent level of confidence. Some change in its coefficient's time series became more apparent, beginning in the 1964 water year at this site. The selected annual seasonal patterns depicted in figure 7 typify pre- (1957) versus post- (1968) reservoir conditions.

The significant time-series changes in all three harmonic coefficients for the Colorado River at Lees Ferry, as previously discussed, coincide with the construction of Glen Canyon Reservoir upstream from the measurement site. 
Table 8.--Trend-analysis summary, long-term records, Upper Colorado River Basin

\begin{tabular}{|c|c|c|c|c|c|}
\hline \multirow{2}{*}{$\begin{array}{c}\text { USGS } \\
\text { Station no. }{ }^{1}\end{array}$} & \multirow{2}{*}{$\begin{array}{l}\text { Number of } \\
\text { years }^{2}\end{array}$} & \multicolumn{4}{|c|}{$\begin{array}{l}\text { Level of significance of trend } \\
\text { Harmonic coefficient }\end{array}$} \\
\hline & & $\mathrm{A}$ & $\mathrm{C}$ & $M$ & $A+M$ \\
\hline 09306500 & 26 & $3-$ & 0 & 4- & $5-$ \\
\hline 09071100 & 32 & 0 & $2+$ & $4+$ & $3+$ \\
\hline 09093700 & 8 & 0 & 0 & $1+$ & 0 \\
\hline 09095500 & 33 & $2-$ & 0 & $5-$ & $2-$ \\
\hline 09152500 & 31 & 4- & 0 & 1 & $3-$ \\
\hline 09163530 & 14 & 0 & 0 & 0 & 0 \\
\hline 09180000 & 26 & 0 & 0 & 0 & 0 \\
\hline 09180500 & 32 & 0 & 0 & 0 & 0 \\
\hline 09355500 & 15 & $5-$ & 4- & $1-$ & $5-$ \\
\hline 09364500 & 23 & 0 & 0 & 0 & 0 \\
\hline 09365000 & 18 & 0 & 0 & 5- & $2-$ \\
\hline 09368000 & 23 & 4- & $1-$ & $2-$ & $3-$ \\
\hline 09379500 & 35 & $1+$ & 0 & 0 & $2+$ \\
\hline 09209400 & 13 & 0 & 0 & $3-$ & $1-$ \\
\hline 09211200 & 9 & $1+$ & $1-$ & $1+$ & $2+$ \\
\hline 09217000 & 31 & $1-$ & $2-$ & 0 & 0 \\
\hline 09234500 & 24 & $5-$ & $3-$ & $1-$ & 4- \\
\hline 09261000 & 30 & 3- & 0 & 0 & $2-$ \\
\hline 09302000 & 23 & 0 & 0 & $3+$ & $3+$ \\
\hline 09307000 & 11 & 0 & 0 & 0 & $1-$ \\
\hline 09314500 & 23 & $1-$ & $1+$ & $2-$ & $3-$ \\
\hline 09315000 & 33 & 0 & $4+$ & 0 & 0 \\
\hline 09328500 & 27 & 0 & $2+$ & 0 & 0 \\
\hline 09251000 & 30 & 0 & $2+$ & $1-$ & 0 \\
\hline $09259950 *$ & 19 & $3+$ & 0 & $3+$ & $3+$ \\
\hline $09260000 *$ & 7 & 0 & $3+$ & $1-$ & 0 \\
\hline 09380000 & 32 & $5-$ & $5-$ & $5-$ & $5-$ \\
\hline
\end{tabular}

${ }^{1}$ See supplementary information and text for station descriptions.

${ }^{2}$ See table 1 . Stations with fewer than 8 years of record were omitted.

${ }^{3}$ See Conover (1971) and Steele and others (1974);

Levels of significance: $1=80$ percent

$2=90$ percent

$3=95$ percent

$4=98$ percent

$5=99$ percent

Sign after ranking indicates direction of trend.

*Combined record for purpose of trend analysis. 
It was coincidental that the time-series changes in annual watertemperature patterns in several cases cited in the Upper Colorado River Basin occurred around 1963-64. Evaluation of the observed shifts in seasonal water-temperature patterns for the case studies evaluates possible future impacts that may occur in rivers for which reservoirs have been proposed. It should be emphasized that the observed effects below reservoirs can vary with the type of reservoir release and the distance downstream from the impoundment structure.

\section{Piceance Basin--A Specific Study Area}

Extensive oil-shale development is anticipated in the Piceance basin, with recently projected production levels as great as 1.7 million barrels per day (Colorado Department of Natural Resources, 1979). Among environmental concerns are the changes in quality and flow conditions in streams draining areas impacted by the development. As part of an extensive analysis of ambient conditions, a total of 83 daily water-temperature records at 14 sites in the basin (see supplemental information section at the end of the report) have been collected during the 1975-81 water-year period. These records were analyzed using the previously described harmonic-analysis approach; resultant coefficients depicting annual seasonal patterns are summarized in table 9.

Stream drainage areas for the measurement sites in this special study area were smaller than those main-stem and tributary sites analyzed previously. Site locations are given in figure 8 ; drainage areas range from 22.3 to 1,632 square kilometers.

In general, standard errors of estimate were small (less than $2^{\circ} \mathrm{C}$ ), and percent annual variability explained by the harmonic function was large (greater than 80 percent) (table 9). Given that a maximum of 7 years of record was available at a given site, no formal analysis of possible trends was attempted.

As examples of regional water-temperature patterns, 1979 harmonicfunction results are depicted in figure 9 for selected sites in the Piceance basin. Amplitude coefficients for the Parachute Creek and Roan Creek sites were small, considering the lower elevations of the measurement sites compared with the Piceance Creek and Yellow Creek sites. For the main-stem Piceance Creek sites (stations 09306007 Piceance Creek below Rio Blanco, Colo.; 09306061 Piceance Creek above Hunter Creek, near Rio Blanco, Colo.; and 09306222 Piceance Creek at White River, Colo.), an increasing stream-reach trend of harmonic-mean temperatures in a downstream direction was apparent (table 9 and fig. 9). 


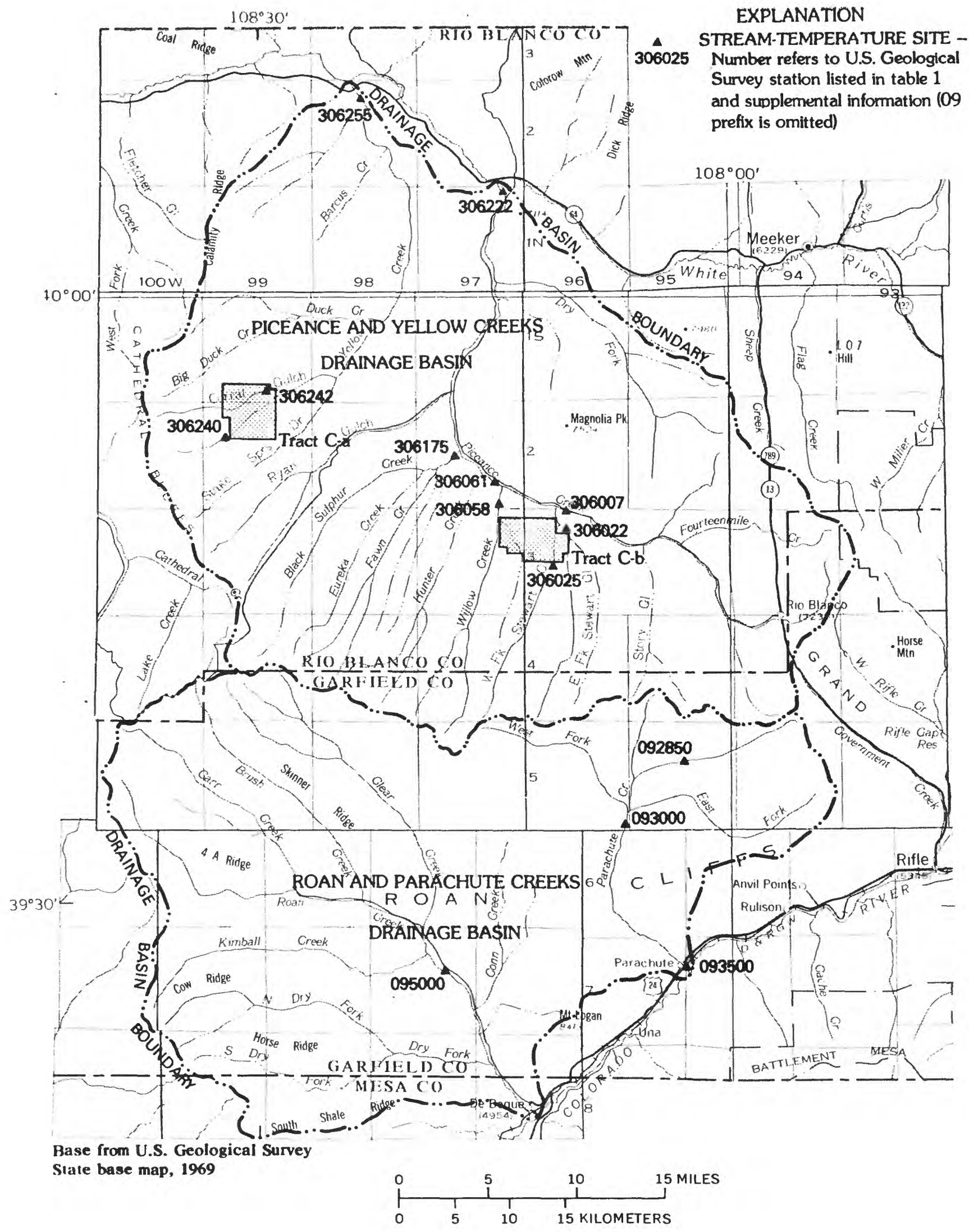


Table 9.--Harmonic-function coefficients, Piceance basin

$[$ NO $=$ number of daily values; $\mathrm{SE}=$ standard error of estimate;

PVAR = percent variance explained by the harmonic function.]

\begin{tabular}{|c|c|c|c|c|c|c|c|}
\hline \multirow[b]{2}{*}{ Station no. ${ }^{2}$} & \multirow[b]{2}{*}{ Year } & \multicolumn{4}{|c|}{ Harmonic coefficients ${ }^{1}$} & \multirow[b]{2}{*}{ SE } & \multirow[b]{2}{*}{ PVAR } \\
\hline & & NO & A & $\mathrm{C}$ & M & & \\
\hline 9092850 & 1977 & 285 & 8.07 & 2.66 & 5.77 & 1.35 & 94 \\
\hline 9092850 & 1978 & 229 & 7.18 & 2.73 & 6.85 & 1.32 & 74 \\
\hline 9092850 & 1979 & 293 & 7.70 & 2.70 & 5.74 & 1.46 & 99 \\
\hline 9092850 & 1980 & 268 & 7.79 & 2.74 & 6.54 & 1.43 & 88 \\
\hline 9092850 & 1981 & 316 & 7.79 & 2.79 & 6.51 & 1.52 & 89 \\
\hline 9093000 & 1975 & 166 & 5.68 & 2.49 & 7.11 & 1.22 & 81 \\
\hline 9093000 & 1976 & 360 & 6.30 & 2.77 & 7.72 & 1.68 & 87 \\
\hline 9093000 & 1977 & 185 & 8.39 & 2.88 & 6.44 & 1.44 & 90 \\
\hline 9093000 & 1978 & 282 & 4.67 & 3.07 & 7.88 & 1.52 & 82 \\
\hline 9093000 & 1979 & 352 & 4.41 & 2.76 & 6.35 & 1.30 & 85 \\
\hline 9093000 & 1980 & 366 & 3.26 & 2.72 & 6.82 & .95 & 81 \\
\hline 9093500 & 1975 & 243 & 6.76 & 2.38 & 11.31 & 1.91 & 87 \\
\hline 9093500 & 1976 & 324 & 7.17 & 2.79 & 11.34 & 1.64 & 89 \\
\hline 9093500 & 1977 & 266 & 9.64 & 2.81 & 10.95 & 1.54 & 95 \\
\hline 9093500. & 1978 & 214 & 6.98 & 2.81 & 10.38 & 2.03 & 87 \\
\hline 9093500 & 1979 & 229 & 5.94 & 2.67 & 9.20 & 1.73 & 83 \\
\hline 9093500 & 1980 & 303 & 6.34 & 2.53 & 9.58 & 1.60 & 76 \\
\hline 9095000 & 1975 & 231 & 6.16 & 2.58 & 9.60 & 1.58 & 87 \\
\hline 9095000 & 1976 & 341 & 7.57 & 2.92 & 9.73 & 1.33 & 94 \\
\hline 9095000 & 1977 & 176 & 10.06 & 2.97 & 11.82 & 1.37 & 96 \\
\hline 9095000 & 1978 & 349 & 6.54 & 2.78 & 9.72 & 1.48 & 90 \\
\hline 9095000. & 1979 & 362 & 6.62 & 2.74 & 8.51 & 1.42 & 91 \\
\hline 9095000 & 1980 & 312 & 6.76 & 2.81 & 9.65 & 1.63 & 99 \\
\hline 9306007 & 1975 & 159 & 7.21 & 2.89 & 7.79 & 1.56 & 92 \\
\hline 9306007 & 1976 & 255 & 7.62 & 2.86 & 8.42 & 1.38 & 93 \\
\hline 9306007. & 1977 & 307 & 9.35 & 2.86 & 8.20 & 1.42 & 95 \\
\hline 7306007 & 1978 & 296 & 6.66 & 2.80 & 8.15 & 1.39 & 72 \\
\hline 9306007. & 1979 & 285 & 6.66 & 2.89 & 8.00 & 1.27 & 92 \\
\hline 9306007 & 1980 & 241 & 6.55 & 2,83 & 7.69 & 1.38 & 70 \\
\hline 9300007 & 1981 & 349 & 7.52 & 2.90 & 9.19 & 1.38 & 92 \\
\hline 9306022 & 1975 & 122 & 3.72 & 3.13 & $7 \cdot 33$ & 1.07 & 85 \\
\hline 9306022. & 1976 & 306 & 3.00 & 3.01 & 7.87 & .69 & 90 \\
\hline 9306022. & 1977 & 253 & 3.72 & 2.91 & 8.11 & .81 & 71 \\
\hline 9306022 & 1578 & 255 & 3.21 & 3.04 & 8.21 & .94 & 82 \\
\hline 9306022 . & 1979 & 250 & 3.57 & 3.13 & 7.93 & .91 & 97 \\
\hline 9300022 & 1980 & 307 & 3.17 & 2.96 & 7.90 & .76 & 87 \\
\hline 9306022 & 1991 & 259 & 2.83 & 2.76 & 8.40 & .72 & 97 \\
\hline
\end{tabular}


Table 9.--Harmonic-function coefficients, Piceance basin--Continued

\begin{tabular}{|c|c|c|c|c|c|c|c|}
\hline \multirow[b]{2}{*}{ Station no. ${ }^{2}$} & \multicolumn{7}{|c|}{ Harmonic coefficients ${ }^{1}$} \\
\hline & Year & NO & A & C & M & SE & PVAR \\
\hline 9306025 . & 1975 & 124 & 11.32 & 2.84 & 7.23 & 2.24 & 60 \\
\hline 9306025 & 1976 & 38 & 11.07 & 3.22 & 9.03 & 2.83 & 86 \\
\hline $930605 B$ & 1975 & 161 & 5.91 & 2.94 & 7.66 & 1.98 & 77 \\
\hline 9306058. & 1976 & 143 & 4.53 & 2.55 & 5.00 & 2.17 & 63 \\
\hline $930605 B$ & 1977 & 182 & 7.12 & 2.93 & b. 87 & 1.33 & 90 \\
\hline 9306059 & 1978 & 181 & 6.45 & 3.09 & 8.41 & 1.62 & 89 \\
\hline 9306058 & 1979 & 193 & 7.04 & 2.92 & 6.89 & 1.43 & 88 \\
\hline 9306058 & 1980 & 266 & 6.51 & 2.95 & 7.76 & 1.27 & 89 \\
\hline 9306058. & 1981 & 302 & 7.01 & 2.94 & 8.60 & 1.50 & 87 \\
\hline 9306061. & 1975 & 125 & 5.62 & 2.53 & 8.46 & 2.03 & 79 \\
\hline 9306061. & 1976 & 280 & 6.53 & 2.82 & 8.38 & 1.31 & 91 \\
\hline 9306061. & 1977 & 146 & 8.42 & 2.88 & 8.25 & 1.10 & 94 \\
\hline 9306061. & 1978 & 333 & 6.56 & 2.81 & 8.89 & 1.53 & 90 \\
\hline 9306061. & 1979 & 222 & 5.98 & 2.78 & 8.78 & 1.53 & 85 \\
\hline 9306061. & 1980 & 295 & 6.51 & 2.86 & 8.33 & 1.27 & 92 \\
\hline 9306061. & 1981 & 330 & 6.80 & 2.85 & 9.19 & $1.3 B$ & 92 \\
\hline 9306175. & 1975 & 190 & 4.61 & 2.33 & 10.00 & 1.45 & 79 \\
\hline 9306175 & 1976 & 184 & 5.45 & 2.90 & 9.53 & .97 & 87 \\
\hline 9306175 & 1977 & 353 & 6.46 & 2.87 & 8.24 & 1.33 & 92 \\
\hline 9301175. & 1978 & 361 & 5.25 & 2.94 & 9.12 & 1.34 & $8 B$ \\
\hline 9308175 . & 1979 & 352 & 5.61 & 2.91 & 8.89 & 1.52 & 87 \\
\hline 9306175. & 1980 & 325 & 5.82 & 2.90 & 7.95 & 1.20 & 85 \\
\hline 9306175. & 1981 & 339 & 5.92 & 2.92 & 8.24 & 1.18 & 95 \\
\hline 9306222. & 1976 & 237 & 9.78 & 2.95 & 9.17 & 1.85 & 91 \\
\hline 9306222. & 1977 & 169 & 10.73 & 2.87 & 8.60 & 2.44 & 73 \\
\hline 9306222. & 1978 & 349 & 9.58 & 2.91 & 9.94 & 1.92 & 92 \\
\hline 9306222. & 1979 & 309 & 9.51 & 2.89 & 9.32 & 1.35 & 92 \\
\hline 9306222. & 1980 & 313 & 9.58 & 2.91 & 9.12 & 1.53 & 99 \\
\hline 9306222 & 1981 & 352 & 9.69 & 2.90 & 10.21 & 1.64 & 93 \\
\hline 9306235. & 1977 & 90 & 9.13 & 2.80 & 5.60 & 2.27 & 83 \\
\hline 9306235 & 1978 & 139 & 9.09 & 2.84 & 7.41 & 1.31 & 90 \\
\hline 9306235 & 1979 & 272 & 6.76 & 2.88 & 7.26 & 1.40 & 91 \\
\hline 9306242. & 1975 & 223 & 2.29 & 2.33 & 9.84 & 1.32 & 63 \\
\hline 9306242. & 1976 & 234 & 3.25 & 2.74 & 9.14 & 1.65 & 53 \\
\hline 9306242. & 1977 & 292 & 3.00 & 2.79 & 8.93 & 1.45 & 69 \\
\hline 9306242 & 1978 & 335 & 5.27 & 2.85 & 10.49 & 1.76 & 81 \\
\hline 5306242. & 1979 & 356 & 5.81 & 3.16 & 12.82 & 1.84 & 83 \\
\hline
\end{tabular}


Table 9.--Harmonic-function coefficients, Piceance basin--Continued

\begin{tabular}{ccccccccc}
\hline & \multicolumn{9}{c}{ Harmonic coefficients } & & \\
Station no. & & Year & NO & A & C & M & SE & PVAR \\
\hline 9306242. & 1980 & 340 & 3.57 & 2.82 & 9.30 & 1.32 & 68 \\
9306242. & 1981 & 357 & 2.52 & 2.87 & 9.64 & .62 & 88 \\
9306255. & 1975 & 183 & 8.60 & 2.74 & 9.15 & 2.64 & 77 \\
9306255. & 1976 & 182 & 9.98 & 2.99 & 8.80 & 2.20 & 88 \\
9306255. & 1977 & 308 & 10.33 & 2.92 & 8.66 & 1.74 & 94 \\
9306255. & 1978 & 304 & 10.13 & 2.90 & 10.40 & 2.05 & 92 \\
9306255. & 1979 & 248 & 10.85 & 2.90 & 8.92 & 1.70 & 93 \\
9306255. & 1980 & 291 & 9.61 & 2.96 & 8.96 & 1.56 & 87 \\
9306255. & 1981 & 340 & 10.26 & 2.95 & 10.11 & 1.83 & 92 \\
\hline
\end{tabular}

${ }^{1}$ See equation (1), p. 2 of text.

${ }^{2}$ See supplemental information for station description. 


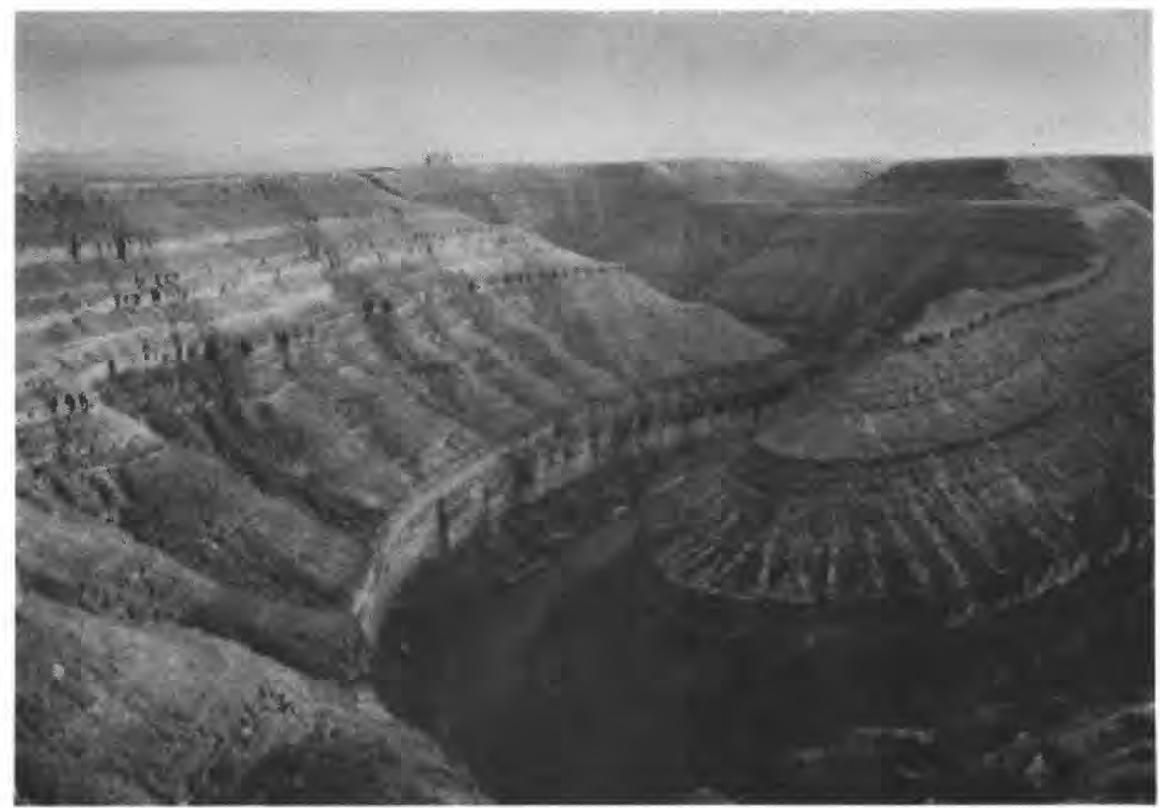

Goosenecks of the San Juan River, Upper Colorado River Basin, looking southeast near Mexican Hat, Utah.

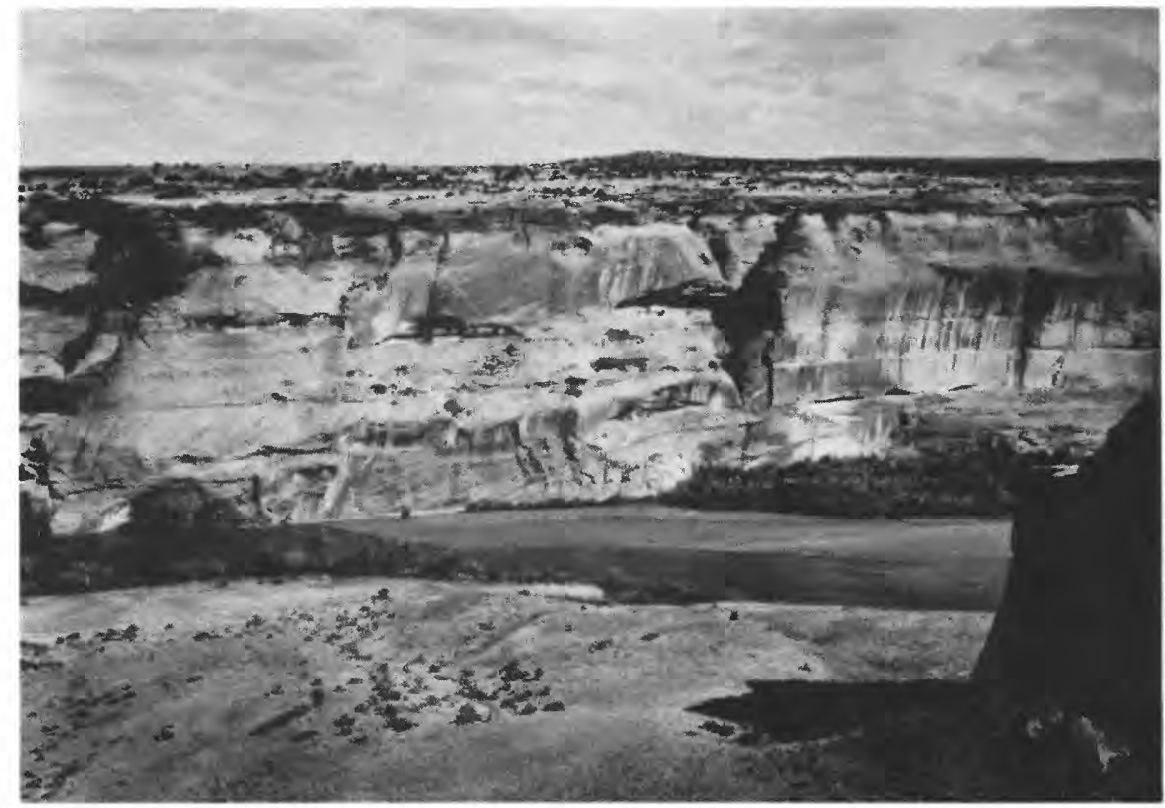

Canyon de Chelly and Chinle Creek, Upper Colorado River Basin, looking east near Chinle, Arizona. 


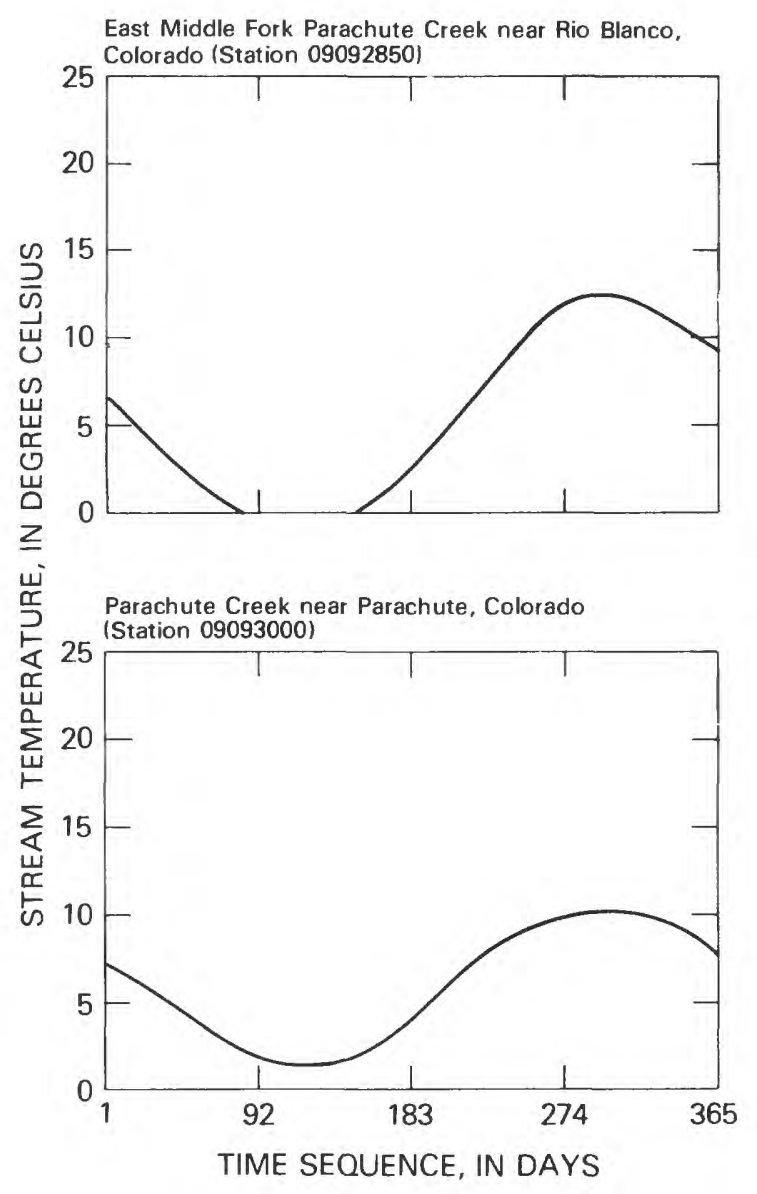

Parachute Creek at Parachute, Colorado (Station 09093500)

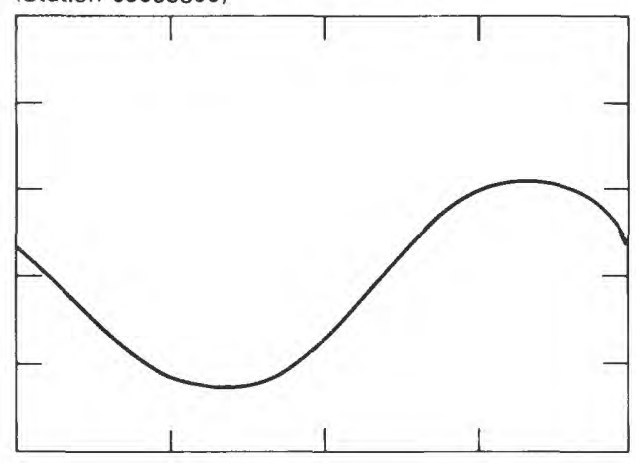

Roan Creek near DeBeque, Colorado (Station 09095000)

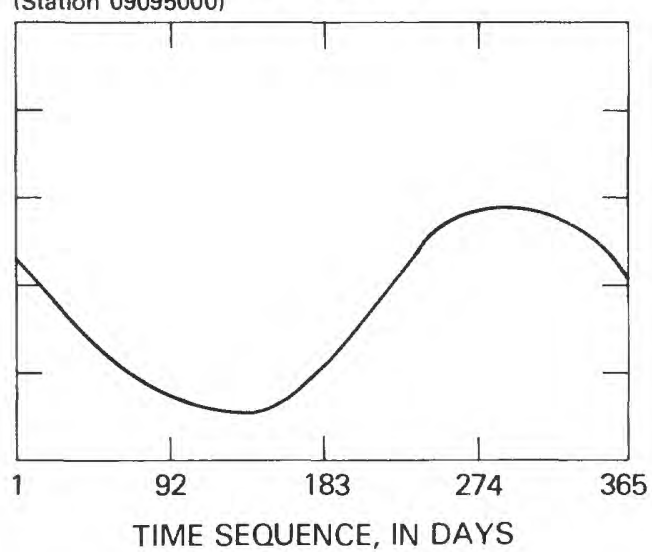

Figure 9.--Seasonal water-temperature patterns at selected sites, Piceance basin, 1979 water year. 


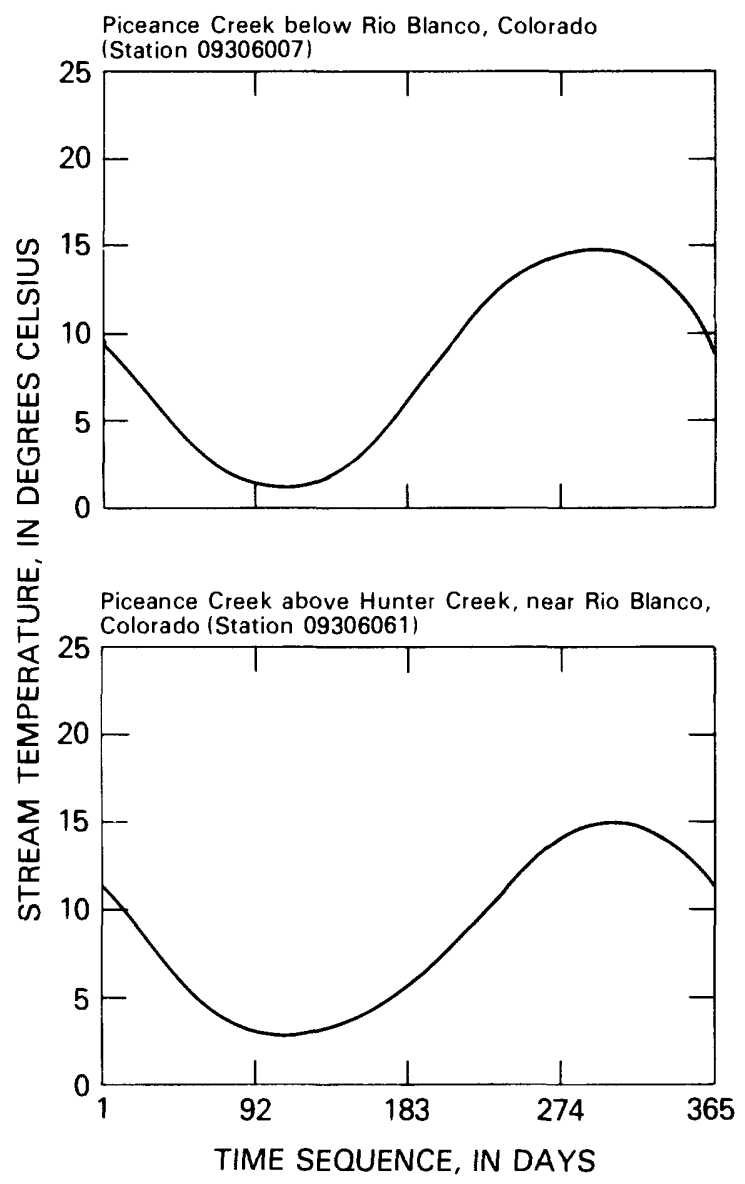

Piceance Creek at White River, Colorado (Station 09306222)

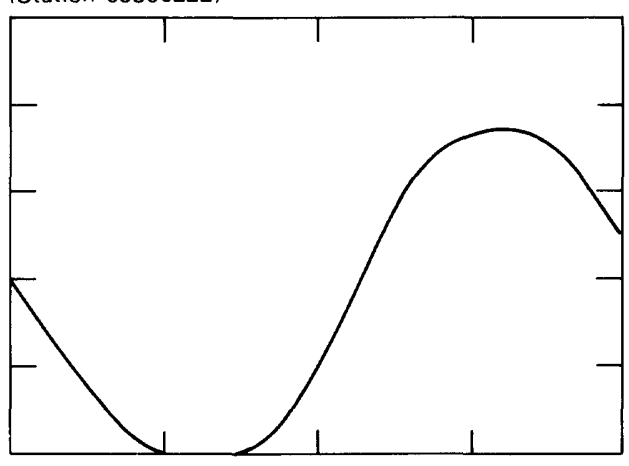

Yellow Creek near White River, Colorado Yellow Creek near
(Station 09306255)

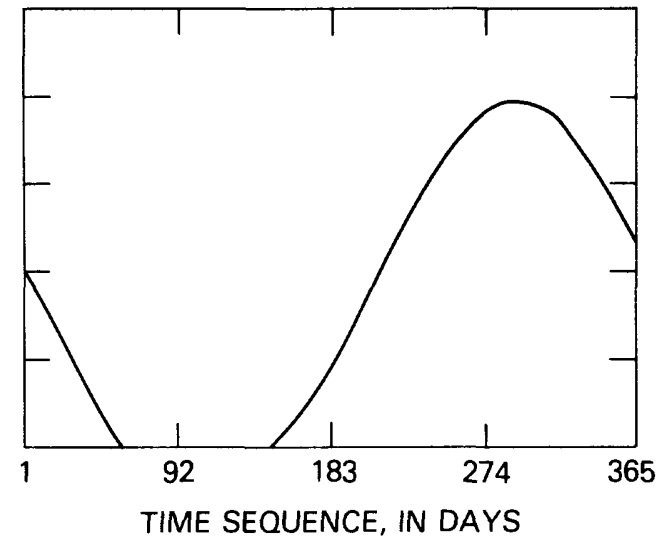

Figure 9.--Seasonal water-temperature patterns at selected sites, Piceance basin, 1979 water year--Continued. 


\section{SUMMARY}

Simple-harmonic functions were used to characterize annual-seasonal patterns of water temperature at numerous measurement sites on main-stem streams and major tributaries in the Upper Colorado River Basin and on smaller streams in the Piceance basin. Daily water-temperature records were evaluated using this technique. A total of 668 station years of daily record was available at 36 sites in the Upper Colorado River Basin; 83 station years of daily record was available at 14 sites in the Piceance basin. Most records contain missing days or gaps in measurements; this technique helps to overcome these data deficiencies and provides a set of coefficients that could be evaluated both areally and temporally.

Comparison of observed discrete water-temperature measurements with a harmonic function, whose coefficients were determined directly from these data or by some indirect means, results in a time-series trace of harmonic residuals (that is, differences between observed and estimated values). Through analysis of these residual values, possible causes can be sought for prolonged periods of positive or negative residuals that may reflect deviations from a standardized seasonal pattern.

Unless short-term monitoring of water temperature is required by legislative mandate or some current purpose, little justification exists for perpetuating a daily-measurement frequency. Related previous studies (Gilroy and Steele, 1972; Steele, 1978; Wentz and Steele, 1980) have shown that minimal information on annual variability is lost by reducing measurement frequency to an approximate monthly pattern. For example, in cases where the measurement site coincides with a streamflow-gaging station, measurements then could be made by field personnel servicing the stage recorders on an intermittent schedule.

Admittedly, water-temperature measurements are relatively inexpensive in terms of time, instruments, and manpower, relative to most other water-quality variables. However, when data processing, publication, data file-storage, and quality-control factors are considered, costs begin to add up. A need to identify specific information needs and desired accuracies of statistical measurements of water temperature is inherent in any review to justify continuance of daily measurements.

In this study, possible benefits of generating long-term watertemperature records have been demonstrated in several cases, where upstream surface-water impoundments were placed "on-line" within the available period of historical record. In these instances, annual seasonal patterns frequently changed, and the resultant annual series of harmonic coefficients were evaluated statistically to assess the observed changes. From these documented case studies, one could anticipate what changes would occur for certain forms of releases for proposed reservoirs. Impacts on fish and aquatic life could be assessed beforehand, which might affect future design criteria for reservoir-release structures. This impact-evaluation feature of the proposed data-analysis procedure is felt to have general applicability. 
In the Piceance basin, water-temperature records were of relatively short duration (2 to 7 years). However, a stronger case should be made to justify continuation of daily measurements of this variable. Otherwise, discrete intermittent measurements taken in conjunction with collecting water-quality samples or servicing stage recorders may be adequate for the intended uses of the information.

In summary, harmonic analysis has been a useful technique for evaluating historical water-temperature records. Use of harmonic functions for estimation purposes provides several cost-saving features and standard comparison capabilities for a more efficient network design and operation.

\section{SELECTED REFERENCES}

Briggs, J. C., and Ficke, J. F., 1978, Quality of rivers of the United States, 1975 water year--Based on the National Stream Quality Accounting Network (NASQAN): U.S. Geological Survey Open-File Report 78-200, May, 474 p.

Clement, R. W., 1978, Temperature in streams of New Mexico for the period October 1964 to September 1969: U.S. Geological Survey Water-Resources Investigations 78-140, 35 p.; available from U.S. Department of Commerce, National Technical Information Service, Springfield, VA 22161, as PB-299 360 .

Colorado Department of Natural Resources, 1979, Upper Colorado River Basin 13(a) assessment - The availability of water for oil shale and coal gasification development in the Upper Colorado River Basin: Summary report submitted to U.S. Water Resources Council, October, IV.

Collings, M. R., 1969, Temperature analysis of a stream: U.S. Geological Survey Professional Paper 650-B, p. B174-B179.

Conover, W. J., 1971, Practical nonparametric statistics: New York, John Wiley, $462 \mathrm{p}$.

Gilroy, E. J., and Steele, T. D., 1972, An analysis of sampling frequency alternatives for fitting a daily stream temperature model: Tucson, Ariz., International Symposium on Uncertainties in Hydrologic and Water Resource Systems, Proceedings, v. 2, p. 594-608.

Hawkinson, R. O., Ficke, J. F., and Saindon, L. G., 1977, Quality of rivers in the United States, 1974 water year--Based on the National Stream Accounting Network (NASQAN): U.S. Geological Survey Open-File Report 77-151, $199 \mathrm{p}$.

Hirsch, R. M., Slack, J. R., and Smith, R. A., 1982, Techniques of trend analysis for monthly water quality data: Water Resources Research, v. 18 , no. 1, February, p. 107-121.

Hutchinson, N. E., compiler, 1975, WATSTORE user's guide: U.S. Geological Survey Open-File Report 75-426, v. 1, August, chap. 4, sec. B.

Iorns, W. V., Hembree, C. H., and Oakland, G. L., 1965, Water resources of the Upper Colorado River Basin--Technical report: U.S. Geological Survey Professional Paper 441, 370 p.

Lowham, H. W., 1978, An analysis of stream temperatures, Green River basin, Wyoming: U.S. Geological Survey Water-Resources Investigations 78-13, 41 p.; available from U.S. Department of Commerce, National Technical Information Service, Springfield, VA 22161, as PB-284 062. 
Shampine, W. J., 1977, Indiana stream-temperature characteristics: U.S. Geological Survey Water-Resources Investigations 77-6, 61 p.; available from U.S. Department of Commerce, National Technical Information Service, Springfield, VA 22161, as PB-265 346.

Smith, R. A., Hirsch, R. M., and Slack, J. R., 1982, A study of trends on total phosphorus measurements at NASQAN stations: U.S. Geological Survey Water-Supply Paper 2190, 34 p.

Steele, T. D., 1974, Harmonic analysis of stream temperatures: Reston, VA, U.S. Geological Survey Computer Contribution, 246 p.; available from U.S. Department of Commerce, National Technical Information Service, Springfield, VA 22161, as PB-239 016/AS.

Steele, T. D., 1978, A simple-harmonic model for depicting the annual cycle of seasonal temperatures of streams: U.S. Geological Survey Open-File Report 78-155, $36 \mathrm{p}$.

Steele, T. D., 1983, A regional analysis of water quality in major streams of the United States: Symposium on Dissolved Loads of Rivers and Water Quality/Quantity Relationships, Proceedings: Hamburg, West Germany, International Association of Hydrological Sciences, Publication No. 141, p. 131-143.

Steele, T. D., Bauer, D. P., Wentz, D. A., and Warner, J. W., 1979, The Yampa River basin, Colorado and Wyoming--A preview to expanded coal-resource development and its impacts on regional water resources: U.S. Geological Survey Water-Resources Investigations 78-126, 142 p.; available from U.S. Department of Commerce, National Technical Information Service, Springfield, VA 22161 , as PB-300 815 .

Steele, T. D., Gilroy, E. J., and Hawkinson, R. 0., 1974, An assessment of areal and temporal variations in streamflow quality using selected data from the National Stream Quality Accounting Network: U.S. Geological Survey Open-File Report 74-217, 210 p.

Ward, J. C., 1963, Annual variation of stream water temperature: American Society of Civil Engineers, Proceedings, Journal of the Sanitary Engineering Division, v. 89, no. SA6, December, p. 1-16.

Wentz, D. A., and Steele, T. D., 1980, An analysis of stream quality in the Yampa River basin, Colorado and Wyoming: U.S. Geological Survey Water Resources Investigations 80-8, 157 p.; available from U.S. Department of Commerce, National Technical Information Service, Springfield, VA 22161, as $\mathrm{PB}-81108904$. 
SUPPLEMENTAL INFORMATION 
Table 10.--Water-temperature measurement-site descriptions

[mi ${ }^{2}$, square mile; $\mathrm{km}^{2}$, square kilometer]

A. Mainstream and major tributary sites, Upper Colorado River Basin (total of 36 stations).

USGS

Station no.

Station description

Drainage area

$\mathrm{mi}^{2} \quad\left(\mathrm{~km}^{2}\right)$

\begin{tabular}{|c|c|c|c|}
\hline 09304200 & White River above Coal Creek, near Meeker, Colo. & 660 & $(1,710)$ \\
\hline 09304600 & White River at Meeker, Colons & 808 & $(2,090)$ \\
\hline 09304800 & White River below Meeker, Colo- & 1,040 & $(2,690)$ \\
\hline 09306395 & White River near Colorado-Utah State line---- & 3,680 & $(9,530)$ \\
\hline 09306500 & White River near Watson, Utah- & 4,020 & $(10,400)$ \\
\hline 09306900 & White River at mouth, near Ouray, Utah- & 5,120 & $(13,300)$ \\
\hline 09071100 & Colorado River near Glenwood Springs, Colo-..-- & n.a. & -- \\
\hline 090 & Colorado River at Glenwood Springs, Colo-..-- & 4,558 & $(11,800)$ \\
\hline 0908 & Roaring Fork River at Glenwood Springs, Colo---- & 1,451 & $(3,760)$ \\
\hline 09093 & Colorado River near De Beque, Colo- & 7,370 & $(19,100)$ \\
\hline 09095500 & Colorado River near Cameo, Colo-.................. & 8,050 & $(20,800)$ \\
\hline 091525 & Gunnison River near Grand Junction, Colo--.-- & 7,928 & $(20,500)$ \\
\hline 09163 & Colorado River below Colorado-Utah State line--- & 18,034 & $(46,700)$ \\
\hline 00 & Delores River near Cisco, Utah- & 4,580 & $(11,900)$ \\
\hline 00 & Colorado River near Cisco, Utah- & 24,100 & $(62,400)$ \\
\hline 09355500 & San Juan River near Archuleta, N. Mex & 3,260 & $(8,440)$ \\
\hline & an Juan River at Bloomfield, N. Mex & 5,410 & $(14,000)$ \\
\hline 00 & Animas River at Farmington, N. Mex & 1,360 & $(3,520)$ \\
\hline 00 & San Juan River at Farmington, N. Mex & 7,240 & $(18,800)$ \\
\hline 09368000 & San Juan River at Shiprock, N. Mex & 12,900 & $(33,400)$ \\
\hline 08379500 & San Juan River near Bluff, Utah-- & 23,000 & $(59,600)$ \\
\hline & Green River at Warren Bridge, near Daniel, Wyo-- & 468 & $(1,210)$ \\
\hline 092 & Green River near La Barge, Wyo- & 3,190 & $(10$ \\
\hline 0921 & Green River below Fontenelle Reservoir, Wyo---- & 4,280 & $(11,100)$ \\
\hline 09217000 & Green River near Green River, Wyo- & 10,000 & $(25,900)$ \\
\hline 09234500 & Green River near Greendale, Utah- & 15,100 & $(39,100)$ \\
\hline 09261000 & Green River near Jensen, Utah- & 25,400 & $(65,800)$ \\
\hline 09302000 & Duchesne River near Randlett, Utah- & 3,920 & $(10,200)$ \\
\hline 09307000 & Green River near Ouray, Utah & 35,500 & $(91,900)$ \\
\hline 09314500 & Price River near Woodside, Utah & 1,500 & $(3,880)$ \\
\hline 09315000 & Green River at Green River, Utah- & 40,600 & $(105,200)$ \\
\hline 09328500 & San Rafael River near Green River, Utah-...-..- & 1,670 & $(4,330)$ \\
\hline 09251000 & Yampa River near Maybel1, Colo- & 3,410 & $(8,830)$ \\
\hline $09259950 *$ & Little Snake River above Lily, Colo-..-..- & n.a. & -- \\
\hline $09260000 *$ & Little Snake River near Lily, Colo- & 3,730 & $(9,660)$ \\
\hline 09260025 & Yampa River below Little Snake River, Colo--.-- & $8,080 \mathrm{E}$ & $(20,900)$ \\
\hline 09380000 & Colorado River at Lees Ferry, Ariz- & 07,900 & $(279,500)$ \\
\hline
\end{tabular}

*Treated as identical station. 
Table 10.--Water-temperature measurement-site descriptions--Continued

B. Measurement sites, Piceance basin (total of 14 stations)

1. Parachute Creek-Roan Creek Basins (4 stations)

\begin{tabular}{|c|c|c|}
\hline $\begin{array}{l}\text { USGS } \\
\text { Station no. }\end{array}$ & Station description & $\begin{array}{l}\text { Drainage area } \\
\mathrm{mi}^{2} \quad\left(\mathrm{~km}^{2}\right)\end{array}$ \\
\hline 09092850 & $\begin{array}{l}\text { East Middle Fork Parachute Creek } \\
\text { near Rio Blanco, Colo- }\end{array}$ & $(57.2)$ \\
\hline 09093000 & Parachute Creek near Parachute, Colo-.... & $(365)$ \\
\hline 09093500 & Parachute Creek at Parachute, Colon.......... & $(513)$ \\
\hline 09095000 & Roan Creek near De Beque, Colo & $(831)$ \\
\hline
\end{tabular}

2. Piceance Creek-Yellow Creek Basins (10 stations)

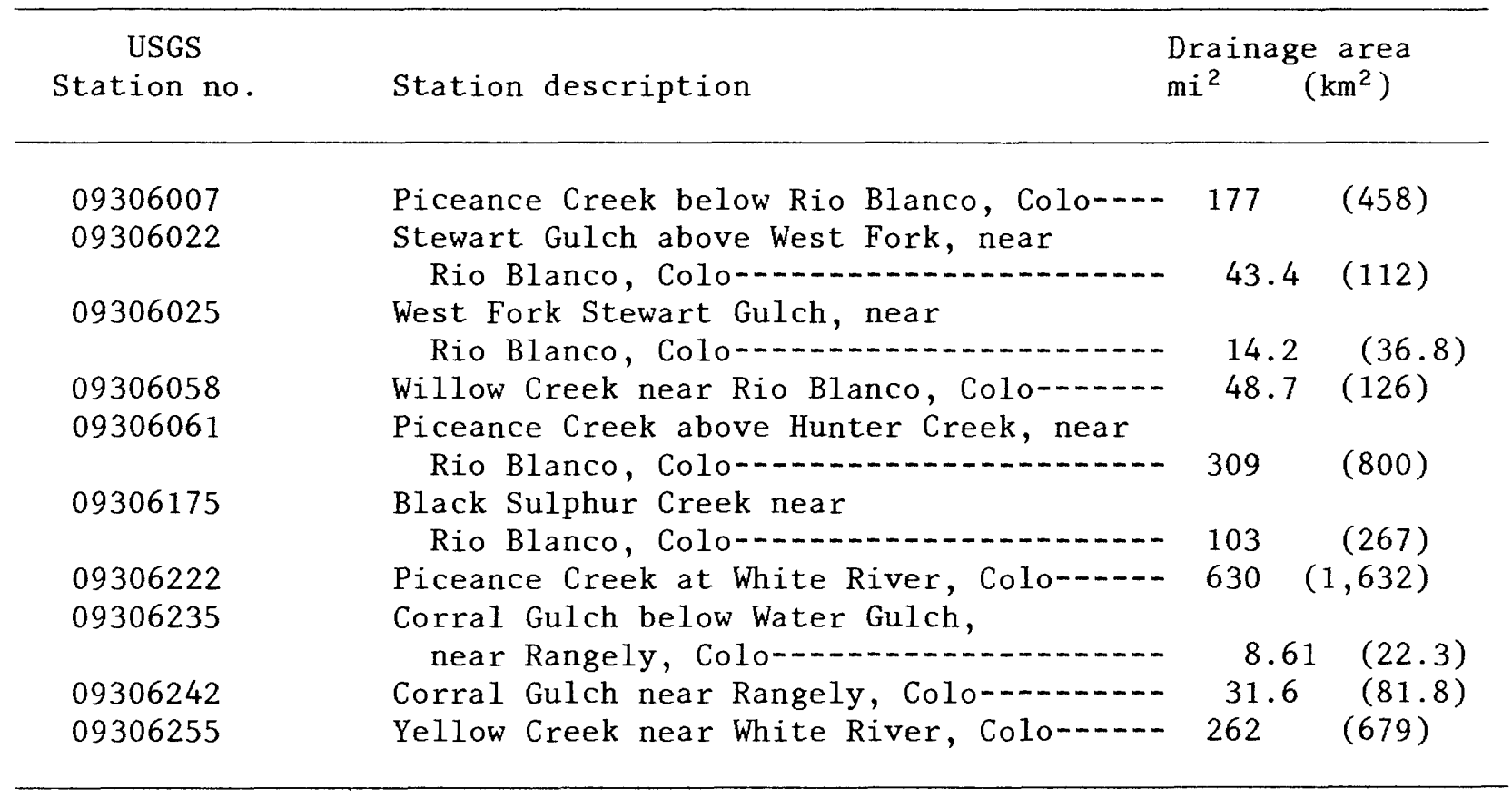

